\title{
The Villafranca catalog of Galactic OB groups II. From Gaia DR2 to EDR3 and ten new systems with $O$ stars
}

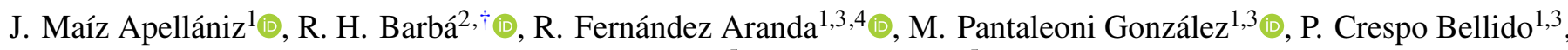 \\ A. Sota ${ }^{5}$, and E. J. Alfaro ${ }^{5}$ \\ ${ }^{1}$ Centro de Astrobiología, CSIC-INTA, Campus ESAC. C. bajo del castillo s/n, 28692 Villanueva de la Cañada, Madrid, Spain \\ e-mail: jmaiz@cab.inta-csic.es \\ 2 Departamento de Astronomía, Universidad de La Serena, Av. Cisternas 1200 Norte, La Serena, Chile \\ 3 Departamento de Astrofísica y Física de la Atmósfera, Universidad Complutense de Madrid, 28040 Madrid, Spain \\ 4 Institute of Astrophysics, University of Crete, 70013 Heraklion, Greece \\ 5 Instituto de Astrofísica de Andalucía-CSIC, Glorieta de la Astronomía s/n, 18008 Granada, Spain
}

Received 4 October 2021 / Accepted 14 October 2021

\begin{abstract}
Context. This is the second paper of a series on Galactic OB groups that uses astrometric and photometric data from Gaia and spectral classifications from the Galactic O-Star Spectroscopic Survey (GOSSS) and the Library of Libraries of Massive-star high-Resolution spectra (LiLiMaRlin). The previous paper was based on the second Gaia data release (DR2). Since then, the early third Gaia data release (EDR3) has appeared with new astrometry and photometry.

Aims. The two aims of this paper are to revise the results for the sample from Paper I using Gaia EDR3 data and to expand the sample of analyzed stellar groups to 26, from Villafranca O-001 to Villafranca O-026.

Methods. We used GOSSS to select Galactic stellar groups with O stars and an updated version of the method in Paper 0 of this series, combining Gaia EDR3 $\mathrm{G}+G_{\mathrm{BP}}+G_{\mathrm{RP}}$ photometry, positions, proper motions, and parallaxes to assign memberships and measure distances. We present 99 spectra from GOSSS and 32 from LiLiMaRlin for stars in the analyzed groups or in their foreground.

Results. We derived distances to the 26 stellar groups with unprecedented precision and accuracy, with total (random plus systematic) uncertainties lower than $1 \%$ for distances within $1 \mathrm{kpc}$ and of $\sim 3 \%$ around $3 \mathrm{kpc}$, which are values almost four times better than for Gaia DR2. We provide homogeneous spectral types for 110 stars and correct a number of errors in the literature, especially for objects in Villafranca O-023 (Orion nebula cluster). For each group, we discuss its membership and present possible runaway and walkaway stars. At least two of the studied groups, Villafranca O-O12 S in NGC 2467 and Villafranca O-014 NW in the North America nebula, are orphan clusters in which the most massive stars have been ejected by dynamical interactions, leaving objects with a capped mass function. The existence of such clusters has important consequences for the study of the initial mass function (IMF), the distribution of supernova explosions across the Galaxy, and the population and dynamics of isolated compact objects. We fit pre-main-sequence (PMS) isochrones to the color-magnitude diagrams (CMDs) of four clusters to derive ages of $2.0 \pm 0.5$ Ma for Villafranca O-026 ( $\sigma$ Orionis cluster), $4 \pm 2 \mathrm{Ma}$ for Villafranca O-016 (NGC 2264), 5.0 $\pm 0.5 \mathrm{Ma}$ for Villafranca O-021 (NGC 2362), and $8 \pm 2 \mathrm{Ma}$ for Villafranca O-024 ( $\gamma$ Velorum cluster).
\end{abstract}

Key words. astrometry - catalogs - Galaxy: structure - open clusters and associations: general - stars: kinematics and dynamics stars: early-type

\section{Introduction}

We are building the Villafranca catalog of Galactic OB stellar groups based on Gaia astrometry and photometry (Prusti et al. 2016) and spectroscopic information from the Galactic O-Star Spectroscopic Survey (GOSSS, Maíz Apellániz et al. 2011) and the Library of Libraries of Massive-Star HighResolution Spectra (LiLiMaRlin, Maíz Apellániz et al. 2019a). In Maíz Apellániz (2019), from now on Villafranca 0, we present the method we used to identify members and measure distances to stellar groups and we applied it to the first two examples. Maíz Apellániz et al. (2020), from now on Villafranca I, describes the project aims and analyzes 16 stellar groups using the second Gaia data release (DR2).

As described in Villafranca I, OB stars are formed in stellar groups that can be either bound (clusters) or unbound (associations or subassociations), with the difference between the two

\footnotetext{
Deceased.
}

categories being difficult to ascertain in some circumstances due to the limitations of the available observational data and to the circumstances of each specific case. Some clusters have double cores, some associations have bound cores at their centers, and the limits between neighbor associations may be poorly defined. This requires each case to be analyzed in detail if we are to understand how massive stars form and how to determine the nature and form of the initial mass function (IMF).

Since Villafranca I was published, the early third Gaia data release (EDR3) has become available (Brown et al. 2021). Gaia EDR3 provides astrometry with reduced uncertainties (Lindegren et al. 2021a) that require the application of a correction to eliminate the parallax bias as a function of position, magnitude, and color (Lindegren et al. 2021b). Once the correction is applied, the remaining systematic uncertainty is of only $10.3 \mu$ as and the angular covariance as a function of separation is well characterized (Maíz Apellániz et al. 2021e), leading to the possibility of significantly improving the distances to stellar groups derived from Gaia DR2 data, such as the ones in 
Villafranca I. Furthermore, Maíz Apellániz et al. (2021e) measured that the Lindegren et al. (2021b) correction worked very well except for the brightest stars, which could be recalibrated using open clusters with bright stars such as the ones in nearby OB groups. This leads to the possibility not only of determining the distances to stellar groups with better accuracies but also of using the same data to improve the Lindegren et al. (2021b) correction with stellar clusters, a possibility suggested in that paper.

In this paper we reanalyze the sample of 16 stellar groups of Villafranca I using Gaia EDR3 data and include ten additional objects. In a companion paper (Maíz Apellániz 2022) we combine the results from those 26 stellar groups with the results for the six globular clusters of Maíz Apellániz et al. (2021e) and for the LMC and SMC of Luri et al. (2021) to derive an improved zero point and a characterization of the external uncertainties for Gaia EDR3 parallaxes. In the next section we present our data and methods, we then describe our results for the individual stellar groups, and we finish our paper with an analysis and an exposition of the next steps in the Villafranca project.

\section{Data and methods}

\subsection{OB groups sample selection}

The long-term goal of the Villafranca project is to include all optically accessible Galactic OB groups with $\mathrm{O}$ stars identified as such from GOSSS and LiLiMaRlin spectroscopy. We plan to achieve it in subsequent papers increasing the sample in steps that add 30-100 O stars each. Villafranca I concentrated on OB groups with very-early O-type stars (O2-O3.5). In this paper, we add ten $\mathrm{OB}$ groups that contain $\mathrm{O}$ stars, are of low-extinction (hence, nearby: all are within $2.5 \mathrm{kpc}$ ), and have a rich stellar population accessible to Gaia. Those criteria yield groups that are at the same time scientifically interesting (being some of the better studied OB groups) and useful for the goals of the companion paper of deriving an improved zero point and characterizing the uncertainties of Gaia EDR3 parallaxes.

Table 1 provides the basic information for the new OB groups in this paper. See Table 1 in Villafranca I for the equivalent information for the original sixteen OB groups. We continue the notation of the previous paper by assigning an ID of the type Villafranca $\mathrm{O}-\mathrm{XXX}$, where the $\mathrm{O}$ refers to the existence of at least an $\mathrm{O}$ star in the group and XXX is a sequential number, with 001 to 016 corresponding to the groups present in Villafranca I and 017 to 026 corresponding to the new ones. For each group we list their common names in the literature, their $\mathrm{O}$, WR, and LBV stars, and the spectral classifications of those. A given name can refer to the stellar group (usually a cluster), a part of it, the associated nebulosity, both the group and the nebulosity, or even be unclear in the literature. For that reason, we include in the text brief descriptions for the possibly confusing cases.

Of the new ten groups, nine are real clusters. The exception is Villafranca O-025 (Trumpler $16 \mathrm{E}$ ), which is a subassociation that is part of Trumpler 16 (Villafranca O-003 is Trumpler $16 \mathrm{~W}$ ), which in turn is part of the Carina nebula association or Car OB1. Car OB1 is the richest OB association within $3 \mathrm{kpc}$ and also includes Villafranca O-002 (Trumpler 14) and several other clusters and non-bound OB groups and we divide it into pieces due to the criterion established in Villafranca I of defining groups in a "cluster scale" (sizes of a few pc), independently of their true nature as clusters, instead of in an "association scale" (sizes of $10 \mathrm{pc}$ or more). Besides the reasons for this described in the previous paper, this condition helps us maintain a small dispersion in real physical distances along the line of sight, which is useful for the purposes of the companion paper on the Gaia EDR3 parallax zero point.

\subsection{Distances to and membership of $O B$ groups}

The supervised method used to determine the distances to and membership of stellar groups has already been described in the previous papers: Campillay et al. (2019), Villafranca 0, and Villafranca I. For the convenience of the reader, we list in Table 2 the notation developed in those references, Maíz Apellániz et al. (2021b), and the companion paper. The method uses a series of filters (defined in Table 2 and listed for each group in Table A.1) to establish an initial membership of $N_{*, 0}$ stars in each group, which is further culled by a 3 -sigma cut in normalized parallax (the corrected parallax minus the group parallax divided by the external uncertainty) that yields $N_{*}$ stars. As explained in Villafranca I, we use restrictive filters as our goal is not to derive a complete sample of group members but rather one in which there are none or very few false positives. That makes the derived distances very robust, as shown by Monte Carlo simulations in Villafranca 0.

Here we describe the differences in the procedure introduced since Villafranca I. The most important (and obvious) one is that we use Gaia EDR3 parallaxes instead of DR2 ones, which are of significantly better quality (Lindegren et al. 2021a). The second most important difference is that we apply a magnitude, color, and Galactic latitude dependent parallax zero point instead of a constant one for all stars (see below for details). A first consequence of this is that the individual (or stellar) parallaxes are corrected before calculating the group parallax, so there is no need to distinguish between uncorrected and corrected group parallaxes, as was done in Villafranca I. A second consequence is that there is no need to add an extra amount in quadrature to the group parallax uncertainty. A third difference regarding the astrometry is that for bright stars we apply the proper motion corrections of Cantat-Gaudin \& Brandt (2021).

When using Gaia DR2 photometry, the $G$ photometry has to be corrected and one has to distinguish between a bright and a faint magnitude range for $G_{\mathrm{BP}}$ (Maíz Apellániz \& Weiler 2018). Those effects are small but it is important to specify whether one uses the catalog magnitudes $(G)$ or the corrected ones $\left(G^{\prime}\right)$. For Gaia EDR3 photometry we have developed similar corrections for $G, G_{\mathrm{BP}}$, and $G_{\mathrm{RP}}$ (Weiler et al., in prep.). We have also substituted the DR2 $d_{\mathrm{CC}}$ parameter by the new $C^{*}$ (Riello et al. 2021). For one group we introduced a maximum corrected $G$ magnitude, $G_{\max }^{\prime}$, to exclude the numerous population of faint field stars in the region, and for two groups we introduced a maximum $\Delta\left(G_{\mathrm{BP}}^{\prime}-G_{\mathrm{RP}}^{\prime}\right)$ in addition to the minimum one. $\Delta\left(G_{\mathrm{BP}}^{\prime}-G_{\mathrm{RP}}^{\prime}\right)$ is the value used as a displacement in color with respect to a reference isochrone used to filter out field stars, see the bottom left plots in Appendix A of Villafranca I where both the reference and displaced isochrones are plotted.

The last filtering step of the process is the 3-sigma cut in normalized parallax, which is dependent on the parallax zero point $Z_{\text {EDR3 }}$ and on the multiplicative constant $k$ used to convert from internal to external parallax uncertainties (Maíz Apellániz et al. 2021e and Table 2). The first effect should be small, as for most sources the variations in $Z_{\mathrm{EDR} 3}$ are small compared to $\sigma_{\text {int }}$. The second effect should also be small unless $k$ is off by $\sim 50 \%$, the amount necessary to shift 2-sigma deviations to 3 sigmas. To address these issues we followed a two-step process. First, we used the zero point of Lindegren et al. (2021b) and the $k$ value of Maíz Apellániz et al. (2021e) to perform an initial selection 
Table 1. Sample of new O-type stellar groups in this paper.

\begin{tabular}{|c|c|c|c|c|}
\hline ID & Other IDs & $\mathrm{O} / \mathrm{WR} / \mathrm{LBV}$ stars & Spectral type & Ref. \\
\hline \multirow[t]{5}{*}{ O-017 } & Heart nebula & HD 15570 & $\mathrm{O} 4$ If & S11 \\
\hline & IC 1805 & HD $15558 \mathrm{~A}^{(\dagger)}$ & $\mathrm{O} 4.5 \mathrm{III}(\mathrm{fc}) \mathrm{p}+\mathrm{O} 8:$ & M19 \\
\hline & Sh 2-190 & HD 15629 & $\mathrm{O} 4.5 \mathrm{~V}((\mathrm{fc}))$ & $\mathrm{S} 11$ \\
\hline & W4 & KM Cas & O9.2 IVn & TW \\
\hline & & At least five others & & \\
\hline \multirow[t]{5}{*}{ O-018 } & Lagoon nebula & 9 Sgr A,B & O3.5 V $\left(\left(f^{*}\right)\right)+$ O5-5.5 V((f)) & $\mathrm{R} 12$ \\
\hline & M8 & HD 165052 & $06.5 V((f)) z+06.5 V((f)) z$ & TW \\
\hline & NGC $6523+6530$ & Herschel 36 & $07.5 \mathrm{~V}+\mathrm{O} 9 \mathrm{~V}+\mathrm{B} 0.5 \mathrm{~V}$ & A10 \\
\hline & Sh $2-25$ & HD 164816 & $09.5 \mathrm{~V}+09.7 \mathrm{~V}$ & TW \\
\hline & W 29 & Possibly two others & & \\
\hline \multirow[t]{6}{*}{ O-019 } & Eagle nebula & HD $168076 \mathrm{~A}, \mathrm{~B}^{(\dagger)}$ & $\mathrm{O} 4 \mathrm{IV}(\mathrm{f})$ & M16 \\
\hline & M16 & $\mathrm{BD}-134923^{(\dagger)}$ & $\mathrm{O} 4 \mathrm{~V}((\mathrm{f}))+\mathrm{O} 7.5 \mathrm{~V}$ & M19 \\
\hline & NGC 6611 & ALS 15360 & O6.5 V((f))z & TW \\
\hline & Sh $2-49$ & HD 168075 & $06.5 \mathrm{~V}((\mathrm{f}))+\mathrm{B} 0-1 \mathrm{~V}$ & S09 \\
\hline & RCW 165 & At least eight others & & \\
\hline & W 37 & & & \\
\hline \multirow[t]{4}{*}{$\mathrm{O}-020$} & Rosette nebula & HD 46223 & $\mathrm{O} 4 \mathrm{~V}((\mathrm{f}))$ & S11 \\
\hline & NGC 2244 & HD 46150 & O5 V((f))z & S11 \\
\hline & Sh $2-275$ & At least four others & & \\
\hline & W 16 & & & \\
\hline \multirow[t]{2}{*}{ O-021 } & NGC 2362 & $\tau \mathrm{CMa} \mathrm{Aa}^{(\dagger)}$ & O9.2 Iab: & M20 \\
\hline & $\tau$ CMa cluster & $\tau \mathrm{CMa} \mathrm{Ab}^{(\dagger)}$ & O9 III & M20 \\
\hline \multirow[t]{4}{*}{$\mathrm{O}-022$} & NGC 6231 & WR 79 & $\mathrm{WC7}+\mathrm{O} 4 \mathrm{III}-\mathrm{I}(\mathrm{f})$ & TW \\
\hline & Collinder 315 & HD $152233 \mathrm{Aa}, \mathrm{Ab}$ & O6 II(f) & S14 \\
\hline & & HD $152248 \mathrm{Aa}, \mathrm{Ab}$ & O7 Iabf + O7 Ib(f) & $\mathrm{S} 14$ \\
\hline & & At least eleven others & & \\
\hline \multirow[t]{3}{*}{ O-023 } & Orion nebula & $\theta^{1}$ Ori $\mathrm{Ca}, \mathrm{Cb}{ }^{(\dagger)}$ & O7 f?p var & M19 \\
\hline & M42+M43 & $\theta^{2}$ Ori $\mathrm{A}^{(\dagger)}$ & O9.2 V + B0.5: V(n) & M19 \\
\hline & NGC 1976+1982 & & & \\
\hline \multirow[t]{2}{*}{ O-024 } & $\gamma$ Vel cluster & $\gamma^{2} \mathrm{Vel}^{(\dagger \dagger)}$ & $\mathrm{WC} 8+\mathrm{O} 7 / 8.5 \mathrm{III}-\mathrm{II}$ & TW \\
\hline & $\begin{array}{l}\text { Pozzo } 1 \\
\text { Brandt } 1\end{array}$ & & & \\
\hline \multirow{4}{*}{$\mathrm{O}-025$} & Trumpler $16 \mathrm{E}$ & $\eta \operatorname{Car}^{(\dagger)}$ & $\mathrm{LBV}+\mathrm{O}:$ & S06 \\
\hline & & HDE 303308 A,B & $\mathrm{O} 4.5 \mathrm{~V}((\mathrm{fc}))$ & $\mathrm{S} 14$ \\
\hline & & CPD -59 2641 & O6 V $((\mathrm{fc}))$ & S14 \\
\hline & Ori cluster & At least ten others & $\mathrm{B} 02 \mathrm{~V}(\mathrm{n})$ & M21 \\
\hline
\end{tabular}

Notes. ${ }^{(\dagger)}$ Bad RUWE or $\sigma_{\text {ext }}>0.1$ mas. ${ }^{(\dagger \dagger)}$ Not in Gaia EDR3.

References. A10: Arias et al. (2010), M16: Maíz Apellániz et al. (2016), M19: Maíz Apellániz et al. (2019b), M20: Maíz Apellániz \& Barbá (2020), M21: Maíz Apellániz et al. (2021c), R12: Rauw et al. (2012), S06: Smith (2006a), S09: Sana et al. (2009), S11: Sota et al. (2011), S14: Sota et al. (2014), TW: This work.

for each group. As described in the companion paper, in that selection there were eleven stars in the 26 Villafranca OB groups that were highly likely to be members but had been excluded based on the normalized parallax criterion. Those eleven stars were manually added to the sample in the companion paper and the whole sample (from the OB groups plus six globular clusters, the $\mathrm{SMC}$, and the $\mathrm{LMC}$ ) was used to derive a new $Z_{\mathrm{EDR} 3}$ and a new $k$. Those two were used in the second step to re-derive the membership of the 26 Villafranca groups and those are the results presented here. We note that the eleven stars that had to be manually added in the companion paper satisfy the normalized parallax criterion in the second step. Also, the $k$ calculated in the companion paper applies strictly only to parallax uncertainties (note that the high-RUWE and 6-parameter solutions cases have a special treatment) but for lack of an alternative we also apply it to proper motion uncertainties, as both are calculated as part of a joint astrometric solution so, to first degree, one would expect $k$ to be similar for both.

Table A.1 lists the field sizes and filters used for the sample and is the equivalent to Table 2 in Villafranca I. For Villafranca O-001 to Villafranca O-016 most of the values are similar to those in the previous paper but some tweaks have been carried out to include a few additional known sources and to uniformize criteria. Villafranca $\mathrm{O}-014$ is a special case that is described below.

In summary, the distances to the stellar groups are based on a careful membership selection supervised with an interactive tool for each individual case using Gaia EDR3 data. 
Table 2. Notation used in this paper.

\begin{tabular}{|c|c|c|}
\hline Symbol & Name & Description/Reference \\
\hline$G$ & Very broad band photometric magnitude & Obtained directly from Gaia EDR3 \\
\hline$G_{\mathrm{BP}}-G_{\mathrm{RP}}$ & Photometric color & Obtained directly from Gaia EDR3 \\
\hline$G^{\prime}$ & Corrected $G$ & Weiler et al. (in prep.) \\
\hline$G_{\mathrm{BP}}^{\prime}$ & Corrected $G_{\mathrm{BP}}$ & Weiler et al. (in prep.) \\
\hline$G_{\mathrm{RP}}^{\prime}$ & Corrected $G_{\mathrm{RP}}$ & Weiler et al. (in prep.) \\
\hline$C^{*}$ & Corrected color excess factor & Eq. (6) in Riello et al. (2021) \\
\hline$\varpi$ & Individual parallax & Obtained directly from Gaia EDR3 \\
\hline RUWE & Renormalized Unit Weight Error & Obtained directly from Gaia EDR3 \\
\hline$Z_{\text {EDR3 }}$ & Parallax zero point & Lindegren et al. (2021b) and companion paper \\
\hline$\varpi_{c}$ & Individual corrected parallax & $\varpi-Z_{\text {EDR3 }}$, Maíz Apellániz et al. (2021e) \\
\hline$\sigma_{\text {int }}$ & $\begin{array}{l}\text { Individual parallax/proper motion internal } \\
\text { uncertainty }\end{array}$ & Obtained directly from Gaia EDR3 \\
\hline$\sigma_{s}$ & $\begin{array}{l}\text { Individual parallax/proper motion systematic } \\
\text { uncertainty }\end{array}$ & $10.3 \mu$ as for parallaxes, Maíz Apellániz et al. (2021e) \\
\hline$k$ & $\begin{array}{l}\text { Multiplicative constant for parallax/proper } \\
\text { motion uncertainties }\end{array}$ & $\begin{array}{l}23 \mu \text { as/a for proper motions, Lindegren et al. (2021a) } \\
1.1-2.7 \text { depending on } G \text {, Maíz Apellániz et al. (2021e) } \\
\text { and companion paper }\end{array}$ \\
\hline$\sigma_{\text {ext }}$ & $\begin{array}{l}\text { Individual parallax/proper motion external } \\
\text { uncertainty }\end{array}$ & $\sqrt{k^{2} \sigma_{\text {int }}^{2}+\sigma_{s}^{2}}$, Maíz Apellániz et al. (2021e) \\
\hline$N_{f}$ & Number of Gaia EDR3 sources in the field & Table A.1, Villafranca I \\
\hline$\alpha, \delta$ & Filtering center J2000 Equatorial coordinates & Table A.1, Villafranca I \\
\hline$r$ & $\begin{array}{l}\text { Filtering separation from the center in the plane } \\
\text { of the sky }\end{array}$ & Table A.1, Villafranca I \\
\hline$\mu_{\alpha *}, \mu_{\delta}$ & Filtering central proper motion & Table A.1, Villafranca I \\
\hline$r_{\mu}$ & $\begin{array}{l}\text { Filtering separation from the center in proper } \\
\text { motion }\end{array}$ & Table A.1, Villafranca I \\
\hline$\Delta\left(G_{\mathrm{BP}}^{\prime}-G_{\mathrm{RP}}^{\prime}\right)$ & $\begin{array}{l}\text { Filtering color displacement with respect to } \\
\text { isochrone }\end{array}$ & Table A.1, Villafranca I \\
\hline$G_{\max }^{\prime}$ & Filtering maximum corrected $G$ magnitude & Table A.1 \\
\hline$N_{*, 0}$ & $\begin{array}{l}\text { Number of stars in group before normalized par- } \\
\text { allax filtering }\end{array}$ & Table A.2, Villafranca I \\
\hline$N_{*}$ & $\begin{array}{l}\text { Number of stars in group after normalized par- } \\
\text { allax filtering }\end{array}$ & Table A.2, Villafranca I \\
\hline$t_{\varpi}$ & Normalized $\chi^{2}$ test for the group parallax & Table A.2, Villafranca 0 \\
\hline$t_{\mu_{\alpha *}}$ & $\begin{array}{l}\text { Normalized } \chi^{2} \text { test for the group proper motion } \\
\text { in } \alpha\end{array}$ & Table A.2, Villafranca 0 \\
\hline$t_{\mu_{\delta}}$ & $\begin{array}{l}\text { Normalized } \chi^{2} \text { test for the group proper motion } \\
\text { in } \delta\end{array}$ & Table A.2, Villafranca 0 \\
\hline$\varpi_{g}$ & Group parallax & Table A.2, Eq. (5) in Maíz Apellániz et al. (2021e) \\
\hline$\sigma_{\varpi_{g}}$ & Group parallax uncertainty & Table A.2, Eq. (7) in Maíz Apellániz et al. (2021e) \\
\hline$\mu_{\alpha *, g}$ & Group proper motion in $\alpha$ & Table A.2, Villafranca 1 \\
\hline$\mu_{\delta, g}$ & Group proper motion in $\delta$ & Table A.2, Villafranca 1 \\
\hline$d$ & Group distance & $\begin{array}{l}\text { Table A.2, Maíz Apellániz (2001, 2005), } \\
\text { Maíz Apellániz et al. (2008) }\end{array}$ \\
\hline
\end{tabular}

The final individual parallaxes use the zero point derived in the companion paper. The distances use the Galactic disk prior of Maíz Apellániz (2001, 2005) with the Maíz Apellániz et al. (2008) parameters but they are quite insensitive to the choice of prior (Pantaleoni González et al. 2021).

\subsection{Spectral classifications}

As we did in Villafranca I, we use GOSSS and LiLiMaRlin spectral classifications whenever possible and literature ones otherwise to spectroscopically describe the most relevant stars in the OB groups. The reader is referred to the GOSSS (Sota et al. 2011, 2014; Maíz Apellániz et al. 2016) and LiLiMaRlin (Maíz Apellániz et al. 2019a,b) papers for details on how they are obtained and processed. In some cases, we present new GOSSS and LiLiMaRlin spectra and spectral classifications. LiLiMaRlin spectra are degraded to a spectral resolution of 2500 in order to compare them with the GOSSS standards. The new GOSSS spectral classifications are given in Tables A.3 and the LiLiMaRlin classifications in Table A.4. The new GOSSS spectrograms are shown in Figs. A.1 to A.17 and the LiLiMaRlin spectrograms in Figs. A.18 to A.23. 


\subsection{Literature distances}

In Villafranca I we did an extensive literature search for distances to Villafranca O-001 through Villafranca O-016 with the goal of comparing our values with those of other methods and with other Gaia DR2 distances. Having established such comparison, in this paper we concentrate on determining the changes in precision between the new Gaia EDR3 distances and the previous Gaia DR2 ones. Nevertheless, for the new stellar groups (Villafranca O-017 through Villafranca O-026) we also make comparisons with some selected literature distances.

\section{Results}

In this section we describe our results for the 26 stellar groups in the sample. The calculated parameters are given in Table A.2. We first briefly cover the objects in the Villafranca I sample. As those groups were extensively discussed in that paper, we simply indicate the most significative changes between Gaia DR2 and EDR3 and present some new GOSSS and LiLiMaRlin results. For the 10 new objects we present a more extensive discussion of each.

\subsection{Sample from Villafranca I}

\subsubsection{The distant region of the Carina-Sagittarius arm}

NGC 3603 (Villafranca O-001) and Gum 35 (Villafranca O-006) were the two most distant groups in Villafranca I. It was mentioned there that they could be physically related if they were at similar distances, as they are close in the sky and have similar extinctions. The uncertainties are much lower now and the results are still consistent with a very similar distance for the two around $7 \mathrm{kpc}$. Therefore, it is possible that the two are part of a previously unrecognized distant $\mathrm{OB}$ association.

For Villafranca O-001 we present a LiLiMaRlin spectrum of the B1.5 Ia supergiant NGC 3603 HST-5 (a.k.a. Sher 25), see Taylor et al. (2014) for the original data. For Villafranca O-006 the only new spectrum is for ALS 18552 , which is just outside the search radius for the cluster but is most likely a cluster member. It is a SB2 system with spectral types O6.5 IV((f)) + B0: V. We have detected a typo in the Villafranca O-006 subsection in Villafranca I: ALS 2063 was correctly identified in Table 1 but was misnamed as ALS 2067 in the text.

\subsubsection{The Carina nebula association}

With the Gaia EDR3 results, Trumpler 14 (Villafranca O-002) and Trumpler $16 \mathrm{~W}$ (Villafranca O-003) have parallaxes still consistent with being at the same distance but now the uncertainties are much lower, $\sim 60 \mathrm{pc}$. The distance is also consistent with the geometric value for $\eta$ Car of $2350 \pm 50$ pc of Smith (2006b). Below we analyze the case of the Trumpler $16 \mathrm{E}$ (Villafranca O-025) subassociation.

We present in Fig. A.1 a new GOSSS spectrum for HD 93129 B with a spectral classification of O3.5 V((fc))z, where the c suffix (Walborn et al. 2010) has been added thanks to a cleaner spatial separation with the A component. In Fig. A.18 we show a clean velocity separation of HD 93161 A into two components, O7 V((f)) and O9 IV, using LiLiMaRlin data. In Figs. A.1 and A.2 we present the spectra for eight earlyB stars in Villafranca O-002 and one in Villafranca O-003.
The most interesting one is ALS 15203 (or CPD -582608 B, note it is not selected as a Villafranca O-002 member by the algorithm because it has a RUWE of 1.69), a previously unrecognized $\mathrm{B}+\mathrm{B}+\mathrm{B} \mathrm{SB} 3$ system with a visual companion, ALS 15204 (or CPD -582608 A), which is an O+O SB2 system (Maíz Apellániz et al. 2016), a good example of the highorder multiplicity achieved by some massive stars. Another B+B SB2 system is ALS 15219 . One object in Villafranca O-002, ALS 15863, is one of the eleven stars included in the second step of the membership selection process (see above).

\subsubsection{The nearby region of the Carina-Sagittarius arm}

Two Villafranca I groups are located at similar distances in the closest region of the Carina-Sagittarius arm: Pismis 24 (Villafranca O-005) and M17 (Villafranca O-009). With Gaia EDR3 data we derive similar distances for both despite being $\sim 22^{\circ}$ apart in the sky.

Figure A.2 includes a spectrogram of the interesting star ALS 19692, located in the halo of Villafranca O-005 (i.e., just outside the search radius) and with a borderline RUWE of 1.40. The star has a variable spectrum with N IV $\lambda 4058$ in emission and $\mathrm{N} v \lambda \lambda 4604,4640$ in absorption that indicates the presence of a very early-type star and that is incompatible with the observed He I $\lambda 4471 / \mathrm{He}$ II $\lambda 4542$ ratio if it were a single star (in Maíz Apellániz et al. (2016) we classified it as O5.5 IV(f)). The spectrum must be composite despite the non-detection of double lines and we assign it an (uncertain) O3: III(f) + O7: III((f)) classification. If confirmed, the primary would be a new member of the rare $\mathrm{O} 3$ subtype.

Figure A.3 includes four O stars in the Villafranca O-009 field of view that had no prior GOSSS classifications but with different degrees of certainty regarding the group membership. $\mathrm{BD}-164818$ is classified as $09.5 \mathrm{~V}$ and had been identified as O8.5 V by Crampton et al. (1978) but it has a very large RUWE and other indications of poor astrometry in Gaia EDR3, so the adscription to M17 is unclear. ALS 19612 is a clear member and is classified here as $06.5 \mathrm{~V}((\mathrm{f})) \mathrm{z}$ with GOSSS data (it had previously received an O6: V classification by Crampton et al. 1978). ALS 19611 also has poor Gaia EDR3 astrometry which does not allow us to confirm its Villafranca O-009 membership and a relatively noisy GOSSS spectrogram yields an O7: V(n) spectral classification (Hoffmeister et al. 2008 gave O6 V). Finally, ALS 19608 is a borderline exclusion by the membership algorithm (normalized parallax of 3.27) with a GOSSS spectrogram that yields O9.5 V (Hoffmeister et al. 2008 gave O9 V).

\subsubsection{Cygnus OB2}

In Villafranca I we analyzed the two clusters in Cyg OB2, Bica 1 (Villafranca O-007) and Bica 2 (Villafranca O-008). With the Gaia EDR3 parallaxes, they have moved slightly closer and their measurements are consistent with the two being at same distance.

Figure A.2 includes three stars in Cyg OB2 without previous GOSSS spectral classifications but analyzed by Kiminki et al. (2007). One of them, ALS 15 128, is in Villafranca O-007 and was classified as O8 V by Kiminki et al. (2007). Here we give it a slightly earlier classification of $07.5 \mathrm{Vz}$. The other two, ALS 15123 and Cyg OB2-23, are in Villafranca O-008 and their previous classifications were $\mathrm{O} 9 \mathrm{~V}$ in both cases. The GOSSS classifications are slightly later: $09.5 \mathrm{~V}(\mathrm{n})$ and $09.5 \mathrm{~V}$, respectively. 


\subsubsection{Villafranca $\mathrm{O}-010=\mathrm{NGC} 6193$}

We present spectral types for three early-B stars in Villafranca $\mathrm{O}-010$ in Fig. A.3. HD 150041 is a group member relatively far from the core, so it could have been born in an earlier stellar generation. We classify it as B0 II, the same spectral type as in Feast et al. (1955). The other two stars, CPD -48 8705 and CPD -48 8704, are B dwarfs.

\subsubsection{Villafranca $\mathrm{O}-011=$ Berkeley 90}

In this group it is worth mentioning three stars. 2MASS J20350798+4649321 was classified as O8 V by Marco \& Negueruela (2017), who noted that its IPHAS photometry indicated $\mathrm{H} \alpha$ emission. In our blue violet spectrum (Fig. A.4) there is also incipient $\mathrm{H} \beta$ and $\mathrm{N}$ III $\lambda \lambda \lambda 4634,4641,4642$ emission. We tentatively assign it the spectral type O8 IV((f))e and suggest future spectroscopic observations to detect if C III $\lambda \lambda \lambda 4647,4650,4651$ is variable and can appear in emission. If that were the case, it would make this object a member of the rare magnetic Of?p class, of which there are only six known Galactic examples (the last addition being $\theta^{1}$ Ori $\mathrm{Ca}, \mathrm{Cb}$, Maíz Apellániz et al. 2019b). However, 2MASS J20350798+4649321 has a RUWE of 6.73 in Gaia EDR3, so its adscription to the cluster (which has another two O-type systems, Maíz Apellániz et al. 2015b) is likely but tentative at this stage. The second star is ALS 11448 B (or LS III +46 11 B), that was classified as B4 V in Maíz Apellániz et al. (2015a) but not formally included in GOSSS at that time. For the third source, IRAS 20342+4645, we do not have a spectrum but its parallax, location (just outside the search radius), and proper motion suggest that it was ejected from the cluster $\sim 130 \mathrm{ka}$ ago. The source is relatively faint in the optical but very red and becomes brighter than any cluster member in the IR, making it a possible massive pre-main-sequence (PMS) runaway star. This hypothesis requires confirmation, as with the data at hand it could also be a late-type star unrelated to Villafranca O-011.

\subsubsection{Villafranca $\mathrm{O}-012=\mathrm{NGC} 2467$}

For the two components of this double cluster (Villafranca O-012 S or Haffner 18 and Villafranca O-012 N or Haffner 19) we obtain Gaia EDR3 distances with much lower uncertainties than in Villafranca I and confirm that they are physically associated, with a difference of less than one sigma. In Villafranca I we indicated that the two earliest O-type systems, HD 64568 and HD 64315 A,B appear to have been ejected from Villafranca O-012 S in nearly opposite directions $\sim 400 \mathrm{ka}$ ago, a hypothesis that is still consistent with the Gaia EDR3 astrometry. We increased the search radius of Villafranca O-012 S to include the cluster halo located to the south. Doing so, a new $\mathrm{O}$ star is added to the census, CPD -26 2711 (Fig. A.4). We obtain a spectral type of O7 Vz and Lodén (1966) had previously classified it as O7. Another star in Villafranca O-012 S, ALS 832, is an earlytype B with a RUWE of 1.78 that is classified here as B0.5 V(n).

\subsubsection{Villafranca $\mathrm{O}-014=$ North America nebula $=$ Bermuda and Gulf of Mexico clusters}

The most relevant result in Villafranca I regarding Villafranca $\mathrm{O}-014$ is that there is no significant stellar group around the Bajamar star, the main source of ionizing photons of the North America and Pelican nebulae. However, while the paper was being reviewed, another analysis by Kuhn et al. (2020) proposed that the Bajamar star was ejected as a walkaway star (Renzo et al. 2019) from the most significant cluster in the region, their group D located in the North Atlantic Ocean and Pelican regions, to the NW of the star (which can be dubbed the Bermuda cluster, following the geographical nomenclature of the North America nebula). Gaia DR2 astrometry placed the other known O star in the field, HD 199579 (which can be dubbed the Toronto star, following the geographical nomenclature of the North America nebula), beyond the North America nebula. The Toronto star has a GOSSS spectral classification of O6.5 V((f))z but is a spectroscopic binary with a weak companion, likely of early-B type (Williams et al. 2001).

In our analysis here we define two regions in the North America nebula, Villafranca O-014 NW and Villafranca O-014 SE, which correspond to groups D and E, respectively, in Kuhn et al. (2020), the latter being a smaller cluster in the Gulf of Mexico region of the nebula. We derive group parallaxes of $1.254 \pm 0.010$ mas and $1.344 \pm 0.016$ mas for the two regions, respectively, values that are slightly larger than the uncorrected Gaia DR2 ones of Kuhn et al. (2020) but compatible after applying a DR2 zero point of $40 \pm 10 \mu$ as (and similar to the more recent Gaia EDR3 value of Kuhn \& Hillenbrand 2020). The derived distances to Villafranca O-014 NW and Villafranca O$014 \mathrm{SE}$ are $798 \pm 6 \mathrm{pc}$ and $744 \pm 9 \mathrm{pc}$, respectively, confirming the finding by Kuhn et al. (2020) that the young stars in the Gulf of Mexico are located closer to us than the ones in the Bermuda cluster.

In a separate paper (Maíz Apellániz et al. 2022) we analyze the relationship between the Bajamar and Toronto stars with the Bermuda cluster in the light of the Gaia EDR3 data. There, we propose that the two O-type SB2 systems have been ejected as walkaways from Villafranca O-014 NW along with other stars ejected as walkaways or runaways, leaving this group as an "orphan cluster" without its more massive stars due to the dynamical interactions among them (Oh et al. 2015). We also detect three objects, 2MASS J20531694+4424298, 2MASS J20522503+4437562, and Tyc 3179-00756-1 which could be $\mathrm{O}$ or B0 stars (the first two are cluster members and the last one a walkaway star) but for which we do not have spectroscopic confirmation at this point.

We present in Figs. A.4 and A.5 the GOSSS spectra of four stars in this region. $57 \mathrm{Cyg}$ is the brightest object in the field and is a foreground twin SB2 B5 V + B5 V. Tyc 3179-01209 is located in Georgia (i.e., just to the north of Florida) and moving quickly away from Villafranca O-014 NW, at whose position was located $\sim 300 \mathrm{ka}$ ago. Nevertheless, it is somewhat in front of the cluster and its GOSSS spectral type is A0 III so it does not appear to be a young star ejected from Villafranca O014 NW. On the other hand, the last two objects, HD 195965 and HD 201795, are confirmed early-B-type runaway stars that were ejected 1.61 Ma and 1.91 Ma ago, respectively, from the Bermuda cluster (Maíz Apellániz et al. 2022).

\subsubsection{Villafranca $\mathrm{O}-016=\mathrm{NGC} 2264$}

For the two components of this double cluster we measure a distance difference of just 4 pc but with a combined uncertainty of $8 \mathrm{pc}$. Their separation in the plane of the sky $\left(22^{\prime}\right)$ also corresponds to $4 \mathrm{pc}$, so at this point we are on the verge of being able to peek into the 3-D structure of Villafranca O-016, something that may be possible with Gaia DR4. However, we caution that $22^{\prime}$ is dangerously close to the halfamplitude of the checkered pattern in the Gaia EDR3 parallaxes 
(Maíz Apellániz et al. 2021e), so systematic effects will have to be improved in future Gaia data releases to accomplish that goal. In Figs. A.5 and A.6 we present GOSSS spectra for twelve B and one A star in Villafranca O-016, including two B+B SB2 systems, HD 47732 and HD 47755, and one very fast rotator, HDE 261903 . HD 47755 is one of the eleven stars included in the second step of the membership selection process (see above). See Fig. 1 for a Gaia EDR3 CMD of Villafranca O-016 that includes 15 Mon $\mathrm{Aa}, \mathrm{Ab}$ and $\mathrm{B}$ (the latter with an estimated $G_{\mathrm{BP}}-G_{\mathrm{RP}}$ from the photometry of Maíz Apellániz et al. 2019b and the spectral types of Maíz Apellániz et al. 2018a) and the two potential runaways from Villafranca I.

\subsection{New groups}

\subsubsection{Villafranca $\mathrm{O}-017=$ Heart nebula $=I C 1805=$ Sh $2-$ $190=W 4$}

The region formed by the Heart (IC 1805 or W 4), Soul (IC 1848 or W 5), and Fishhead (IC 1795 or W 3) nebulae is the most prominent optical H II complex in the second Galactic quadrant and together they form the Cas OB6 association (Garmany \& Stencel 1992; Carpenter et al. 2000; Megeath et al. 2008). Here we analyze only the stellar group in IC 1805 and leave the other two and their relationship for future Villafranca papers, noting only that IC 1795 is the youngest of the three. The Heart nebula itself is a cavity created by the stellar group at its center. There are nine known O stars in Villafranca O-O17, with the earliest ones being HD 15570 (one of the O4 If spectral classification standards, Maíz Apellániz et al. 2016), HD 15558 A (an SB2 that is part of a complex system with thirteen components in the WDS catalog, Mason et al. 2001, some of them likely chance alignments from the cluster, Maíz Apellániz et al. 2019b), and HD 15629 (O4.5 V((fc)), another spectral classification standard, Maíz Apellániz et al. 2016).

In the GOSSS papers we have published the spectra and spectral types of eight of the nine known O-type systems in Villafranca O-017. The missing one is KM Cas, an O9.2 IVn star (Fig. A.7) that was previously classified as O9.5 V((f)) by Massey et al. (1995) and that is relatively far from the cluster center and close to the western edge of the cavity drawn by the Heart. In Figs. A.7, A.8, and A.18 we also show GOSSS and LiLiMaRlin spectra for seven stars in Villafranca O-017, including one supergiant (BD +60 493), two B+B SB2 systems (BD +60 496 and ALS 7320), and one fast rotator (ALS 15 320).

Villafranca O-017 has a core surrounded by a rich halo. The core itself is not very prominent and the halo is just the central region of the Cas OB6 association, with IC 1795 towards the NW and the more distant IC 1848 towards the SE. The group population is not well differentiated in proper motion from the background disk population around $3-4 \mathrm{kpc}$ that dominates for fainter stars and this manifests in the relatively large number of stars rejected by the normalized parallax criterion. Indeed, it is possible that there is a slight remaining contamination in the final sample that would artificially push the measured distance out but, if present, we estimate that to be a small effect $(\sim 20 \mathrm{pc})$. Of the nine O-type systems, the membership algorithm selects eight, with the missing one (HD 15558 A) rejected due to its large RUWE and $\sigma_{\text {ext }}$, and the additional five not mentioned above being BD +60 497, BD +60 498, BD +60 499, $\mathrm{BD}+60501$, and BD +60 513 .

The previous distance measurements to IC 1805 span a large range of values from $1.45 \mathrm{kpc}$ (Zucker et al. 2020) to $3.79 \mathrm{kpc}$ (Foster \& MacWilliams 2006), to mention only results in the last two decades. Our value of $2075_{-42}^{+44} \mathrm{pc}$ is in between those two and also between the distances of two analyses from isochrone fitting of optical photometry from the last decade: $1.7 \mathrm{kpc}$ of Kharchenko et al. (2012) and $2.4 \mathrm{kpc}$ of Sung et al. (2017). It is also within two sigmas of the two precise maser distances to the nearby IC 1795 (Megeath et al. 2008).

From the positions, parallaxes, and proper motions we detect six potential runaway stars from Villafranca O-017: ALS 7310, 2MASS J02332978+6135238, 2MASS J02344503+6125106, 2MASS J02334807+6142036, 2MASS J02334886+6141045, and 2MASS J02331163+6116585. The first two have published spectral types of B1 V (Ishida 1970) and G7 Ib (Shi \& Hu 1999, possibly a PMS object), respectively.

\subsubsection{Villafranca $\mathrm{O}-018=\mathrm{Lagoon}$ nebula $=\mathrm{M} 8=\mathrm{NGC}$ $6523+6530=$ Sh $2-25=$ W 29}

The Lagoon nebula (M8), one of the most famous H II regions in the sky, contains at least two entries in the NGC catalog, NGC 6523 and NGC 6530, that we will use to refer to two subclusters (the first towards the $\mathrm{W}$ and the second towards the E but see Tothill et al. 2008 for the confusing NGC nomenclature regarding the region, which includes two additional entries) that will be analyzed together here. The earliest type system in M8 is $9 \mathrm{Sgr}$ A,B, which was classified as O4 V((f)) in Maíz Apellániz et al. (2016) but is a low-velocity-separation eccentric SB2 (Fabry et al. 2021) composed of an O3.5 V((f*)) primary and an O5-5.5 V((f)) secondary according to Rauw et al. $(2012)^{1}$. 9 Sgr A,B is located in NGC 6523, the western and brightest part of M8, and is its main source of ionization. Close to it we find Herschel 36, a high-order O-type multiple system (Arias et al. 2010; Sánchez-Bermúdez et al. 2014; Campillay et al. 2019), that is the origin of the Hourglass nebula (Maíz Apellániz et al. 2015c) and is still partially embedded in its primordial cloud, hence having a higher extinction and with a large value of $R_{5495}$ (Maíz Apellániz \& Barbá 2018). Contrary to the hollowed-out appearance of the Heart nebula, M8 is still filled with nebulosity and actively forming stars (Barbá \& Arias 2007). Another two O-type SB2 systems are present in Villafranca O-018, HD 164816 and HD 165052, and discussed below. A fifth O star, HD 164536, is outside the search radius we use for Villafranca O-018 and also has a poor RUWE and a large $\sigma_{\text {ext }}$. A sixth O star, HD 165246 is also outside the search radius.

In Fig. A.18 we present new LiLiMaRlin spectra for HD 164816 and HD 165052. In the first case the new spectral classification is $09.5 \mathrm{~V}+09.7 \mathrm{~V}$, which makes the secondary slightly earlier than in Sota et al. (2014), where we classified it as B0 V. In the second case the new classification is O6 V((f))z + O7 V((f))z, where the primary remains as 06 as in Maíz Apellániz et al. (2016) and the difference between the two components is of one spectral subtype as in Arias et al. (2002) and Ferrero et al. (2013). The slight variations in spectral classifications arise in this case because of the introduction of a new classification criterion (the $\mathrm{z}$ suffix in this case, see Arias et al. 2016), the intrinsic difficulties in disentangling in velocity systems with maximum separations of just $\sim 200 \mathrm{~km} \mathrm{~s}^{-1}$, and the existence of a Struve-Sahade effect (Stickland 1997; Arias et al. 2002; Linder et al. 2007). In addition to the above, in Fig. A.8 we present GOSSS spectra for three B-type stars in Villafranca O-018.

1 The O4 classification is the reason why Villafranca O-018 was not included in Villafranca I. 

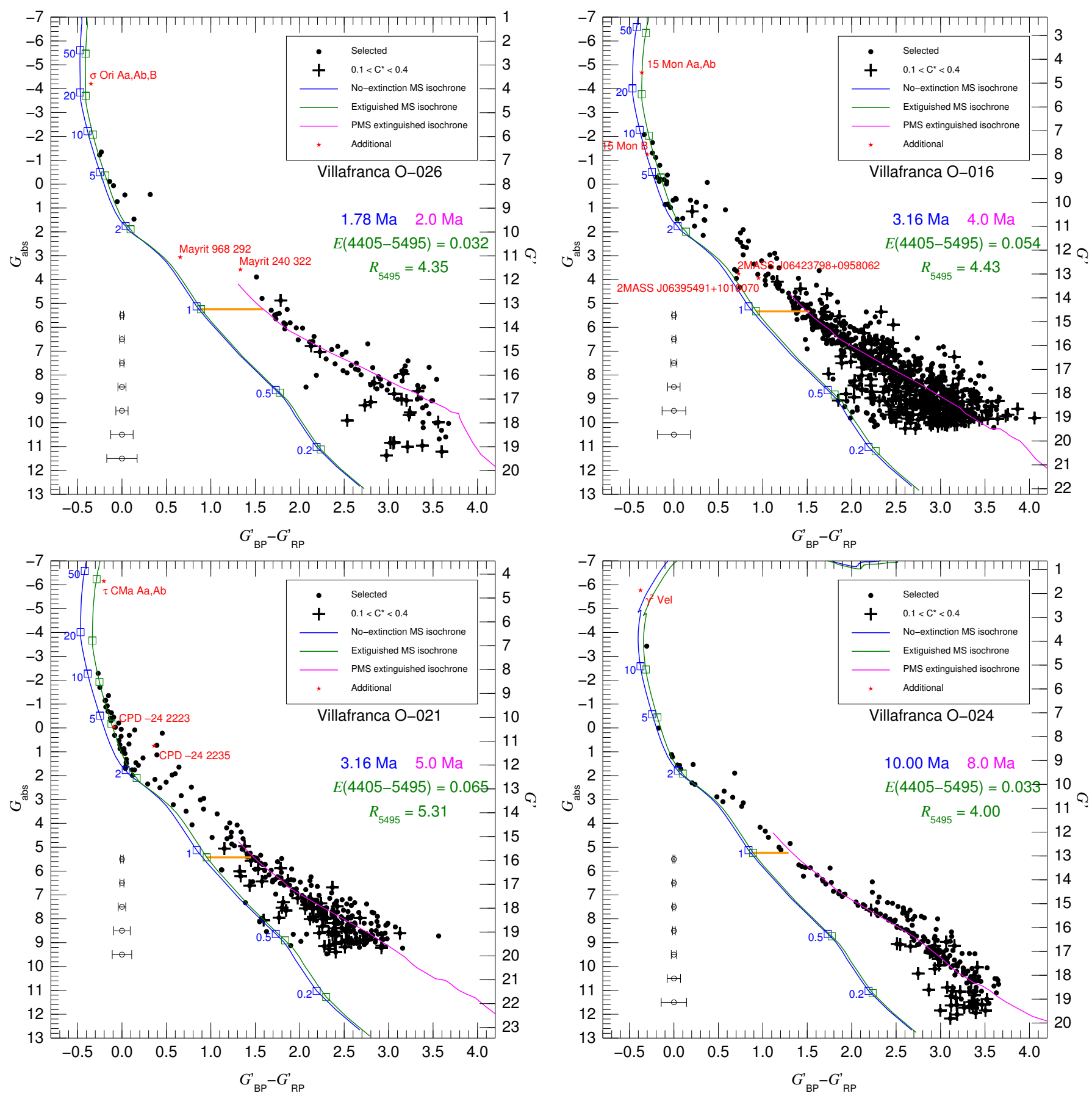

Fig. 1. Gaia EDR3 CMDs for four clusters in this paper: Villafranca O-026 ( $\sigma$ Orionis cluster, top left), Villafranca O-016 (NGC 2264, top right), Villafranca O-021 (NGC 2362, bottom left), and Villafranca O-024 ( $\gamma$ Velorum cluster, bottom right). In each case the sample plotted was selected using less restrictive criteria than for the sample used for the distance (the $\sigma_{\text {ext }}$ restriction was changed to $<0.5$ mas and no RUWE filter was applied). Also, likely additional members and possible walkaways/runaways are plotted and labelled in red. Crosses indicate objects in the sample with likely contaminated $G_{\mathrm{BP}}^{\prime}-G_{\mathrm{RP}}^{\prime}$. The error bars in the lower left quadrant of each plot give typical color uncertainties for different $G_{\mathrm{abs}}$ bins. The blue and green lines show MS Geneva isochrones (Lejeune \& Schaerer 2001) with no extinction and with an average extinction appropriate to the cluster, respectively. Initial masses (in solar units) are labelled along the isochrones and the blue and green text at the right side of each panel gives the age and extinction parameters of the isochrones. The magenta line shows the extinguished PMS isochrone from Baraffe et al. (2015) of the age indicated by the text of the same color at the right side of the panel. The PMS isochrones reach up to $1.4 M_{\odot}$. The orange line marks the gap in $G_{\mathrm{BP}}^{\prime}-G_{\mathrm{RP}}^{\prime}$ between the MS and the PMS at the location of a $1 M_{\odot}$ main-sequence star.

Villafranca O-018 has a peculiar structure. The largest concentration of stars (what may be termed the main core) is the eastern subcluster, NGC 6530, but three of the four O-type systems are in the western subcluster, NGC 6523, the fourth one, HD 165052, is located further east in the halo and if we include
HD 164536 as a fifth O-type member it is even more distant. Nevertheless, it is a very rich OB group (with the second highest $N_{*}$ in Table A.2) and a population that is partially distinguished in proper motion with respect to the dominant background population in Gaia EDR3, located at distances of 3-4kpc. 
The distinction is partial because there is some overlap which translates into a relatively large difference between $N_{*, 0}$ and $N_{*}$. The other factor that helps differentiating the Villafranca O-018 population from the background is its configuration, similar to that of Villafranca O-016 (NGC 2264), in which the extinction associated with the cluster is behind it (i.e., Villafranca O-018 is on the front side of its natal cloud) and the global Gaia EDR3 source density increases with the separation from the center instead of the other way around. That is the reason for introducing a maximum $\Delta\left(G_{\mathrm{BP}}^{\prime}-G_{\mathrm{RP}}^{\prime}\right)$ in Table A. 1 for this group. The membership algorithm selects three of the four O stars, with 9 Sgr A,B excluded only because of its large $\sigma_{\text {ext }}$.

Most recent distance determinations to Villafranca O-018 place it at a distance around $1.3-1.4 \mathrm{kpc}$, see Table 6 in Aidelman et al. (2018) where the authors own measurement is a clear outlier with a value of $2245 \pm 215 \mathrm{pc}$. Another recent distance determination is that of Wright et al. (2019) at $1326_{-69}^{+77} \mathrm{pc}$. Our value here of $1234 \pm 16 \mathrm{pc}$ places it somewhat closer and is in good agreement with our previous determinations of $1.25 \mathrm{kpc}$ (Arias et al. 2006) using photometry processed with CHORIZOS (Maíz Apellániz 2004) and of $1169_{-60}^{+67}$ pc (Campillay et al. 2019) using Gaia DR2 parallaxes. There is one potential runaway star in the Gaia EDR3 data for Villafranca O-018: CPD -24 6114. Also, the O-type HD 165246 may be a walkaway star.

\subsubsection{Villafranca O-019 $=$ Eagle nebula $=M 16=$ NGC 6611 $=$ Sh $2-49=\mathrm{RCW} 165=\mathrm{W} 37$}

The Eagle nebula (M16) is another famous H II region, pictured in the iconic "pillars of creation" HST image, which are the consequence of the different rates of photoevaporation of the molecular gas in the natal cloud as a function of density (Hester et al. 1996). Similarly to Villafranca O-016 (NGC 2264) and Villafranca O0018 (M8), the H II region is a concave hole in the front wall of a molecular cloud carved by the action of the $\mathrm{O}$ stars, leading to differential (in amount and type) extinction effects as a function of sightline (Fig. 7 in Maíz Apellániz \& Barbá 2018). Villafranca O-019 hosts a rich population of massive and PMS stars (Oliveira 2008) with at least 12 O-type systems. The two earliest ones are HD 168076 A,B (combined spectral type of O4 IV(f), the visual companion has no resolved spectral type yet, Maíz Apellániz et al. 2016) and BD -13 4923 (an SB2 system with spectral types $\mathrm{O} 4 \mathrm{~V}((\mathrm{f}))+07.5 \mathrm{~V}$, Maíz Apellániz et al 2019b).

In Figs. A.8 and A.9 we present GOSSS spectra for six O-type systems and three B stars in Villafranca O019. In Figs. A.18 and A.19 we present LiLiMaRlin spectra for three of the same six O-type systems. Five of the O-type systems had not appeared in GOSSS or LiLiMaRlin before but all had prior classifications as O-type: BD -13 4930 (O9.7 V here, O9.5 V in Hiltner \& Iriarte 1955), BD -13 4929 (an interesting SB3 system with a classification of $\mathrm{O} 8 \mathrm{~V}+\mathrm{B} 0.5: \mathrm{V}+\mathrm{B} 0.5$ : $\mathrm{V}$ previously identified and similarly classified by Sana et al. 2009), BD -13 4928 (O9.7 IVnn here, O9.5 Vn in Hillenbrand et al. 1993), ALS 4903 (O9 V here, O8.5 V in Hillenbrand et al. 1993), and ALS 15352 (O8 IV here, O8.5 V in Hillenbrand et al. 1993). The sixth O star is ALS 15 360, classified as O7 V((f))z in Maíz Apellániz et al. (2016) and here (with a better S/N spectrogram) as O6.5 V((f))z. The three B stars are ALS 15369, BD -13 4921, and ALS 15363, the latter a peculiar object with narrow and deep lines that could be enriched in $\mathrm{He}$.
Villafranca O-019 is a rich cluster with a well-defined core surrounded by a halo that is easily detected in the Gaia EDR3 data despite the fact that the dominant population in the field has similar proper motions, even more so than for the (relatively) nearby in the plane of the sky Villafranca O-018. The reason is that there are very few Gaia EDR3 sources located beyond the cluster, as the associated molecular gas appears to be thick enough to create an extinction wall that eliminates them from the sample. The OB stars in the sample have a broad range of colors, from $G_{\mathrm{BP}}^{\prime}-G_{\mathrm{RP}}^{\prime}$ around 0.5 for the less extinguished one to values larger than 2.0 for the most extinguished ones identified by spectral type. As noted by Maíz Apellániz \& Barbá (2018), extinction increases as we move from the core towards the NE and it is possible that some additional $\mathrm{O}$ stars remain undetected there. Of the known twelve O-type stars, the algorithm selects eight members: HD 168075, HD 168137 Aa,Ab, BD -13 4927, BD -13 4930, BD -13 4928, ALS 4903, ALS 15352, and ALS 15360. The remaining four (HD 168076 A,B, QR Ser, $\mathrm{BD}-13$ 4929, and BD -13 4923) have RUWE larger than 1.4. One object in Villafranca O-019, ALS 15369, is one of the eleven stars included in the second step of the membership selection process (see above).

The older distance estimations to Villafranca O-019 had a wide range of values from $1.7 \mathrm{kpc}$ to $3.4 \mathrm{kpc}$ (Oliveira 2008) but values in the last two decades cluster in the narrower $1.6-1.9 \mathrm{kpc}$, a trend that follows that of the analysis for the 16 stellar groups in Villafranca I that we presented in Table 5 there. Our value of $1697_{-30}^{+31} \mathrm{pc}$ is close to the middle of the latter range. The value is within one sigma of that of the nearby Villafranca O-009 $\left(2.4^{\circ}\right.$ or $70 \mathrm{pc}$ ), so M16 and M17 are likely to be physically related. Villafranca O-019 also has a rich stellar population but the relative orientation of the stellar group with respect to its natal molecular cloud (sideways rather than in front) significantly increases the internal extinction and makes it harder to study. We detect two possible walkaways/runaways in Villafranca O-019: 2MASS J18185057-1347338 (G5 III, Evans et al. 2005), and 2MASS J18182988-1336248 (B9 III, Evans et al. 2005).

\subsubsection{Villafranca $\mathrm{O}-020=$ Rosette nebula $=$ NGC $2244=$ Sh $2-275=$ W 16}

The Rosette nebula (NGC 2244) is the prototype H II region that has been hollowed out by a central cluster with $\mathrm{O}$ stars, a stage that shares with Villafranca O-017 (IC 1805) and that takes place after the one in which the HII region is still filled (e.g., Villafranca O-018 and Villafranca O-019). Such a temporal evolution is also seen in more massive clusters (MacKenty et al. 2000; Maíz Apellániz et al. 2004) but what makes the Rosette nebula special is its excellent circular symmetry in the optical emission lines, which is nevertheless broken in CO and the IR dust emission with an extension towards the SE (Román-Zúñiga \& Lada 2008). Villafranca O-020 contains seven O stars: HD 46223 (the earliest one at O4 V((f)), Sota et al. 2011), HD 46150, HD 46149, HD 46106, HD 46202, HD 46056, and HDE 258691.

We present GOSSS and LiLiMaRlin spectrograms for two $\mathrm{O}$ stars and three B stars in Villafranca O-020 in Figs. A.11 and A.19. HDE 258691 had not appeared in previous GOSSS papers and is here classified as O9.5 V, having been previously identified as O9 V by Turner (1976). HD 46106 was classified as O9.7 III(n) in Sota et al. (2014). We have analyzed ten LiLiMaRlin epochs to determine that the lines (of the primary) move with a peak-to-peak amplitude of $\sim 150 \mathrm{~km} \mathrm{~s}^{-1}$ and we have used the epochs near the extremes to detect the weak (and broad) lines of the secondary, whose peak-to peak 
amplitude appears to be $\sim 400 \mathrm{~km} \mathrm{~s}^{-1}$, identifying this system as an SB2 for the first time. We obtain a spectral classification of $09.7 \mathrm{~V}+\mathrm{B} 2$ : Vn, making this another example of how the combined spectral type of a late-O dwarf and an early-B dwarf appears to be a late-O star of higher luminosity class and of how in such systems the secondary tends to be a fast rotator (Walborn et al. 2014; Maíz Apellániz et al. 2018a, 2021c). One of the B stars, HDE 259135 is an eclipsing SB2 binary (Hensberge et al. 2000) for which we derive a spectral classification of B0 V + B1: V and for which we confirm its membership to Villafranca O-020.

Villafranca O-020 is relatively easy to detect in the Gaia EDR3 proper motions with respect to the dominant background population, located at distances of 3-5 kpc. The selected members form a cluster core that extends towards the SE, similarly to what is seen in $\mathrm{CO}$ and the IR dust emission, with another minor extension towards the SW and a halo around the core. Five of the seven $\mathrm{O}$ stars are selected as members by the algorithm, with the exceptions being HD 46202 and HDE 258691. The first case can be explained by its RUWE of 4.98 and its $\sigma_{\text {ext }}$ of 0.376 mas. HDE 258691 is more interesting: it also has a large RUWE but it is also excluded for being far from the core (indeed, it is outside our search radius). However, its proper motion points away from the cluster core, suggesting it was ejected as a runaway from there 1.0-1.5 Ma ago. One object in Villafranca O020 , HD $46056 \mathrm{~B}$, is one of the eleven stars included in the second step of the membership selection process (see above).

Previous distance measurements to Villafranca O-020 place the cluster at values of $1.4-1.7 \mathrm{kpc}$ (Román-Zúñiga \& Lada 2008), with Gaia DR2 values concentrating at $1.55-1.59 \mathrm{kpc}$ (Cantat-Gaudin et al. 2018; Kuhn et al. 2019; Mužić et al. 2019). Our Gaia EDR3 value of $1421_{-20}^{+21} \mathrm{pc}$ is significantly lower that the latter ones but we note that if we process the Gaia DR2 data for Villafranca O-020 in a manner similar as that in Villafranca I we obtain $1465_{-64}^{+70} \mathrm{pc}$, so at least part of the difference must involve the sample selection and/or the treatment of systematic effects. Nevertheless, the usually reliable distance method of eclipsing binaries yields $1.39 \pm 0.10 \mathrm{kpc}$ for HDE 259135 (Hensberge et al. 2000) which, as we pointed out above, is confirmed as a cluster member. That distance measurement is in excellent agreement with both of our Gaia DR2 and EDR3 values.

\subsubsection{Villafranca $\mathrm{O}-021=\mathrm{NGC} 2362=\tau$ CMa cluster}

NGC 2362 is different from the previous four stellar groups in that it has already dispersed the gas around it. Therefore, it is at an even later morphological evolutionary stage than Villafranca O-017 (IC 1805) or Villafranca O-020 (NGC 2244). Its older age of $\sim 5 \mathrm{Ma}$ (see below) also manifests in most of its PMS population having already lost their circumstellar disks (Dahm 2008). Villafranca O-021 is dominated by its central multiple system, $\tau$ CMa. $\tau$ CMa Aa,Ab is a visual binary with spectral types O9.2 Iab: and O9 III (Maíz Apellániz \& Barbá 2020) but the $\mathrm{Aa}, \mathrm{Ab}$ pair hides two additional components, as one of the $\mathrm{O}$ stars (likely Aa) is an SB1 (Stickland et al. 1998) and there are eclipses with a different period (van Leeuwen \& van Genderen 1997). Those two are the only O stars in the cluster (which has a lower mass than the previous four) and, barring any undetected compact objects, the only two stars to have significantly evolved from the main sequence. The earliest B-type star in Villafranca O-021 is $\tau$ CMa D, with a classification of B0.7 V (Maíz Apellániz \& Barbá 2020). We do not present new GOSSS and LiLiMaRlin spectra for Villafranca O-021, as we recently did it in that paper.

The population of Villafranca O-021 is easily distinguished from the rest of the stars in the Gaia EDR3 field, as it is a rich and centrally concentrated cluster with a proper motion very different from the dominant background population at a distance of $\sim 5 \mathrm{kpc}$ (Gaia peers into the distant outer Galactic disk along this sightline) and with well defined main- and pre-main-sequences in the CMD (Fig. 1). $\tau$ CMa Aa,Ab (a single source in Gaia EDR3) is not selected by the membership algorithm due to its RUWE (and other issues with its astrometry) but the early-type B stars $\tau$ CMa D, CPD -24 2213, and $\tau$ CMa B are. HD 57192 appears to be a foreground object while $\tau$ CMa C (an extremely fast rotator classified as B5 Vnnn in Maíz Apellániz \& Barbá 2020) appears to be a background star. We detect two possible walkaways/runawys from this cluster: CPD -24 2223 and CPD -24 2235.

Dahm (2008) lists prior distances to NGC 2362 that range from 904 pc (Humphreys 1978) to 2090 pc (Johnson \& Morgan 1953). In Maíz Apellániz \& Barbá (2020) we used eight members to derive a Gaia DR2 distance of $1.50_{-0.10}^{+0.12} \mathrm{kpc}$, which is significantly longer than the $1227_{-16}^{+17} \mathrm{pc}$ here and with much worse uncertainties. Analyzing the difference between the two results is instructive for the purposes of understanding results with different Gaia datasets and methods (Fig. 2). The lower uncertainty in this paper is easy to understand: Gaia EDR3 has lower random and (uncorrected) systematic uncertainties and the new sample is significantly larger (116 vs. 8), allowing us to reach the minimum uncertainty allowed by the angular covariance (Maíz Apellániz et al. 2021e). The significant reduction in distance can also be explained: bright, blue stars are the ones most affected by the (non-applied) differential zero point in Gaia DR2 (Fig. 5 in Villafranca I) and all of the eight stars in Maíz Apellániz \& Barbá (2020) happened to be in that category. If instead of using only those eight stars we apply the same selection criteria to the Gaia DR2 data as we have done in this paper, we obtain 108 stars and derive a distance of $1267_{-65}^{+73} \mathrm{pc}$, which is in good agreement with the new Gaia EDR3 value. The lesson here is that systematic effects can be important in Gaia data and need to be accounted for. We have also included a middle panel in Fig. 2 with the result of applying the Lindegren zero point instead of the new one to show how the new solution reduces the uncorrected parallax zero point for the eight bright stars in Maíz Apellániz \& Barbá (2020).

\subsubsection{Villafranca $\mathrm{O}-022=\mathrm{NGC} 6231=$ Collinder 315}

NGC 6231 is the richest (in OB stars) cluster among the ten new groups added to the Villafranca sample in this paper, especially if we consider it as the nucleus of the surrounding Sco OB1 association (but would be surpassed by Car OB 1 or Cyg OB2 if we considered associations rather than clusters within $2.5 \mathrm{kpc}$ ). The cluster is significantly evolved, with the earliest isolated Otype system being HD 152233 Aa,Ab (O6 II(f), Sota et al. 2014) and with a Wolf-Rayet present (WR 79), which has an O star as a companion (see below). From a morphological point of view, it is in an evolutionary state intermediate between that of Villafranca O-020 (NGC 2244) and that of Villafranca O-021 (NGC 2362): the cluster has created a cavity so large around it that the remaining $\mathrm{H} \alpha$ nebulosity is at a long distance and only partially surrounds it (Reipurth 2008). Counting WR 79 (a short period $\mathrm{WC}+\mathrm{O}$ binary system), there are fourteen O-type systems in Villafranca O-022, most of them late-type O dwarfs and giants. There are diverging age measurements for the cluster 

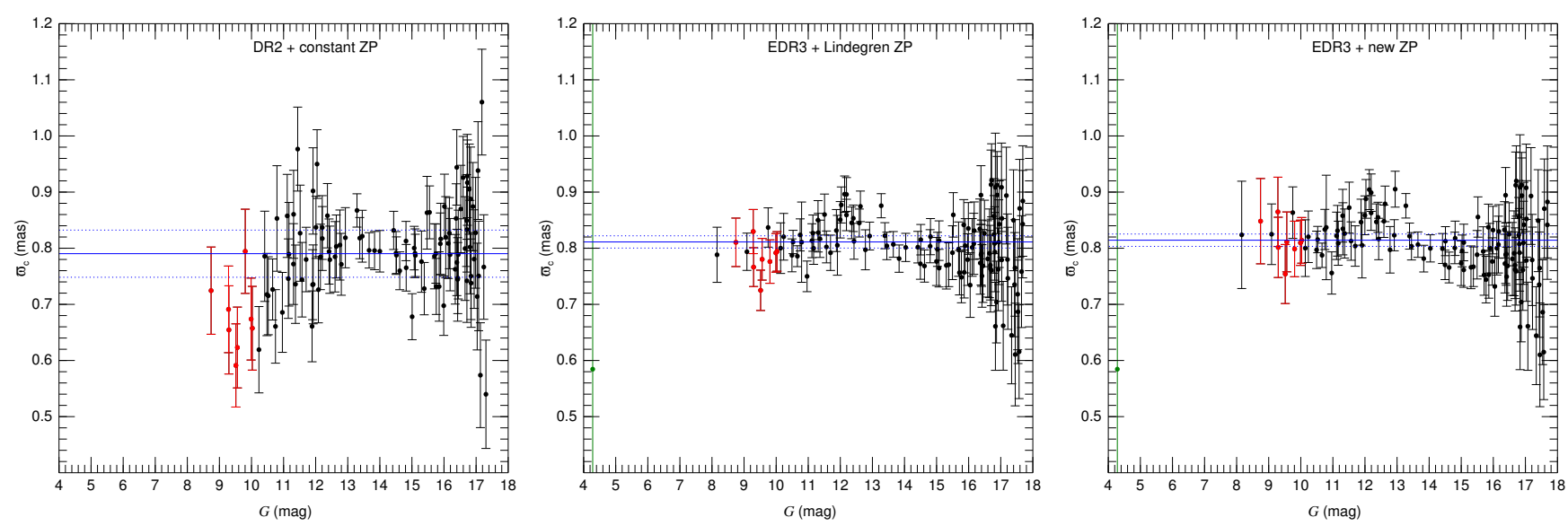

Fig. 2. Corrected individual parallaxes as a function of $G$ for the Villafranca O-021 (NGC 2362) stars selected by the membership algorithm using parallaxes from (left) Gaia DR2 with $Z_{\mathrm{DR} 2}=-40 \mu$ as, (middle) Gaia EDR3 with the Lindegren $Z_{\mathrm{EDR} 3}$ and the $k$ from Maíz Apellániz et al. (2021e), and (right) Gaia EDR3 with the new $Z_{\mathrm{EDR} 3}$ and the $k$ from the companion paper. Red symbols are used for the eight stars used to calculate the distance to the cluster in Maíz Apellániz \& Barbá (2020). The green point with the large error bar near the left edge in the Gaia EDR3 plots corresponds to (the not selected) $\tau \mathrm{CMa} \mathrm{Aa}, \mathrm{Ab}$ (the star is not present in Gaia DR2). The horizontal blue lines show the (corrected) group parallax (with the one sigma range plotted with dotted lines) calculated with each dataset.

(and association), likely due to the presence of different populations and of a real age spread (Reipurth 2008), but the absence of $\mathrm{O}$ dwarfs with subtypes earlier than $\mathrm{O} 8$ indicates that massivestar formation stopped 4-5 Ma ago.

We present GOSSS and LiLiMaRlin spectrograms for one WR, four O-type and twelve B-type stars in Villafranca O-022 in Figs. A.10-A.13, A.19, A.20, and A.21. The WR Catalogue ${ }^{2}$ (Rosslowe \& Crowther 2015) lists a spectral classification of WC7 + O5-8 for WR 79. We show a GOSSS spectrogram of the star in Fig. A.10 that confirms the WC nature of the primary but extends only to $5500 \AA$, which is not enough to assign it a spectral subtype. The equivalent LiLiMaRlin spectrogram in Fig. A.20, on the other hand, extends to $5930 \AA$ and allows us to access the $C$ lines in that region and confirm the WC7 subtype (Crowther et al. 1998). We use our spectral classification software MGB (Maíz Apellániz et al. 2012) to fit the diluted He absorption components in the GOSSS and LiLiMaRlin spectrograms (with He II $\lambda 4542$ significantly more intense than He I 14471) to establish a classification for the secondary of O4 III-I(f). This is earlier than the previous spectral type and is a likely sign that the secondary has been rejuvenated by the mass transfer between the two components.

As for the $\mathrm{O}$ stars with new spectra in Villafranca O-022, the LiLiMaRlin data for HD $152248 \mathrm{Aa}, \mathrm{Ab}$ gives a spectral classification of $\mathrm{O} 7 \mathrm{Ib}(\mathrm{f})+\mathrm{O} 7 \mathrm{Ib}(\mathrm{f})$, which is the same as the GOSSS one in Sota et al. (2014) but downgrading the luminosity class of the primary from Iab to Ib. Another small change takes place for HD 152218, going from O9 IV + B0: V: in Sota et al. (2014) to O8.5 IV + B0 IV here using a LiLiMaRlin spectrogram. Similarly, V1034 Sco goes from O9.2 IV + B1: V in Maíz Apellániz et al. (2016) to O9.5 IV + B1: V here using LiLiMaRlin data. For CPD -41 7733 we detect the weak secondary (Sana et al. 2007) in a LiLiMaRlin spectrogram and assign a classification of O9 IV + B to the system.

The identification of the Villafranca O-022 population in the Gaia EDR3 data is somewhat paradoxical. On the one hand, this is such a rich and concentrated cluster that we obtain the highest value of $N_{\star}$ of all groups. On the other hand, its sightline has low extinction (for its Galactic coordinates) for several kpc

\footnotetext{
2 http://pacrowther.staff.shef.ac.uk/WRcat/
}

(being quite empty of dust until its location and more patchy for longer distances) and there is little change in proper motion as a function of distance, making it hard to distinguish between the cluster and background populations. For that reason, both a maximum $\Delta\left(G_{\mathrm{BP}}^{\prime}-G_{\mathrm{RP}}^{\prime}\right)$ and a $G_{\max }^{\prime}$ are needed in Table A.1 for this group. WR 79 and all of the $\mathrm{O}$ stars in Villafranca O-022 but one are selected by the membership algorithm, the exception being HD 152219 due to its high RUWE and $\sigma_{\text {ext }}$. Another two very bright early-B supergiants, HD 152234 (B0.5 Ia) and HD 152235 (B0.7 Ia) are also excluded by poor-quality astrometry. Three objects in Villafranca O-022, HDE $326331 \mathrm{~A}$, HD $152314 \mathrm{Aa}, \mathrm{Ab}$, and HDE 326330, are among the eleven stars included in the second step of the membership selection process (see above).

The earlier distance determinations to Villafranca O-022 placed the cluster at values between $1.8 \mathrm{kpc}$ and $2.4 \mathrm{kpc}$ (Reipurth 2008) but most determinations after the 1980s placed it somewhat closer. Our value of $1551_{-24}^{+25} \mathrm{pc}$ is not too different from the average value of $\sim 1.6 \mathrm{kpc}$ from the last decades but we note that the two Gaia DR2 determinations by Cantat-Gaudin et al. (2018) and Kuhn et al. (2019) yield somewhat longer distances than our Gaia EDR3 value, as it also happens for Villafranca O-020 (NGC 2244). We also note that the CMD hints of a double sequence for mid-B-to-early-A stars, with one that follows the isochrone that corresponds to the massive stars in the cluster and another with a lower extinction. The second sequence also has a somewhat higher average parallax that corresponds to a $\sim 20 \mathrm{pc}$ difference, so one possible interpretation is that it corresponds to foreground members of Sco OB1, in which case a significant fraction of the extinction we see for Villafranca O-022 would be associated with the cluster itself.

\subsubsection{Villafranca $\mathrm{O}-023=$ Orion nebula cluster $=\mathrm{M} 42+\mathrm{M} 43$ $=$ NGC $1976+1982$}

The Orion nebula is arguably the most famous and studied H II region due to its proximity, low foreground extinction, filled-in morphology, and favorable orientation with respect to the parent molecular cloud (O'Dell et al. 2008). The nebula has the designation M42 (NGC 1976) and the underlying stellar group 
extends to the adjacent Mairan's nebula (M43 or NGC 1982). The cluster is usually called the Orion nebula cluster (ONC) and that is what we will refer to as Villafranca O-023 here. Villafranca O-023 is part of Ori OB1d, the youngest subgroup of the Ori OB1 association (Bally 2008), and has a rich and wellstudied young stellar population (Muench et al. 2008). The core of the ONC is the Trapezium, which receives its name from the shape formed by the four bright systems $\theta^{1}$ Ori $\mathrm{A}+\mathrm{B}+\mathrm{C}+\mathrm{D}$, each one of them a multiple (Karl et al. 2018). In the central region the dominant ionizing source is the O-type system $\theta^{1}$ Ori $\mathrm{Ca}, \mathrm{Cb}$ while further out a second O-type system, $\theta^{2}$ Ori A, also contributes to the mix (O'Dell et al. 2017) and M43 is ionized by its central early-type-B system, NU Ori (Shultz et al. 2019). $\theta^{1}$ Ori $\mathrm{Ca}, \mathrm{Cb}$ is a double (possibly triple) system (Kraus et al. 2009) whose primary (Ca) is a magnetic O7 f?p var star (Maíz Apellániz et al. 2019b). $\theta^{2}$ Ori A is an SB2 system for which in Maíz Apellániz et al. (2019b) we gave a GOSSS classification of O9.2 V + B0.5: V(n) and a LiLiMaRlin classification of $\mathrm{O} 9.5 \mathrm{~V}+\mathrm{B} 0.2$ : $\mathrm{V}$ and also has a faint visual companion.

We present GOSSS and LiLiMaRlin spectrograms for nine B-type stars in Villafranca O-023 in Figs. A.13, A.16, A.21, and A.22. Note that all seven LiLiMaRlin spectrograms have GOSSS equivalents, something that is done in purpose to compare circular aperture échelle vs. long-slit single-order data. Everything else being equal, the first has the advantages of the better original resolution and the higher $\mathrm{S} / \mathrm{N}$ at the (degraded) resolution of the single-order spectrograph. However, the second one has other advantages: easier rectification, possible separation of close components with profile fitting, and (most importantly in this case) better nebular subtraction. Amidst the strong nebulosity of the region, LiLiMaRlin spectra display [O III] $\lambda \lambda 4959,5007$ and Balmer emission lines and, in some cases, partial He I line infilling. Given the existence of conflicting spectral types for some of the famous stars in the region we attempt to provide some clarity by giving details about the spectral classifications below:

- $\theta^{1}$ Ori D (=HD 37023) has a surprisingly broad range of spectral classifications, from O9.5 V: (Slettebak 1963) to B1.5 Vp (Levenhagen \& Leister 2006). Here we classify it as B0 V based on He II $\lambda 4542$ being weaker than Si III $\lambda 4552$ but not by much (if they were similar, the spectral classification would be O9.7, see Sota et al. 2011) and on He II $\lambda 4686$ being stronger than Si III 14552 . Previous classifications as O9.5 or B0.5 may be understandable (but still wrong) based on the diversity of data quality and classification criteria and on the different degrees of nebular contamination. However, anything later than B0.5 is an obvious mistake, given the clear presence of He II absorption lines. Given that this system has at least three components (Karl et al. 2018) and that one of them $(\mathrm{Db})$ could be bright enough to contribute to the spectral classification, do we see signs of its presence in the spectrograms? Indeed, in Fig. A.21 and even more so in Fig. A.13 we see that C II $\lambda 4267$, a line that peaks around B2, is enhanced with respect to what one would expect for a B0 V star. That prompted us to search for variations among the LiLiMaRlin epochs and the result is seen in Fig. 3: O II $\lambda 4254$, which originates in the primary, is always a single line that moves with a peak-to-peak amplitude of $\sim 60 \mathrm{~km} \mathrm{~s}^{-1}$. C II $\lambda 4267$, on the other hand, becomes double with the two components having amplitudes of $\sim 60 \mathrm{~km} \mathrm{~s}^{-1}$ and $\sim 110 \mathrm{~km} \mathrm{~s}^{-1}$ (the Mercator epoch is the one in Fig. A.21 but the double lines are not seen there because of the lower spectral resolution of the plot). Vitrichenko (2002) identified $\theta^{1}$ Ori D as an SB1 (not an SB2, as here) but his velocity

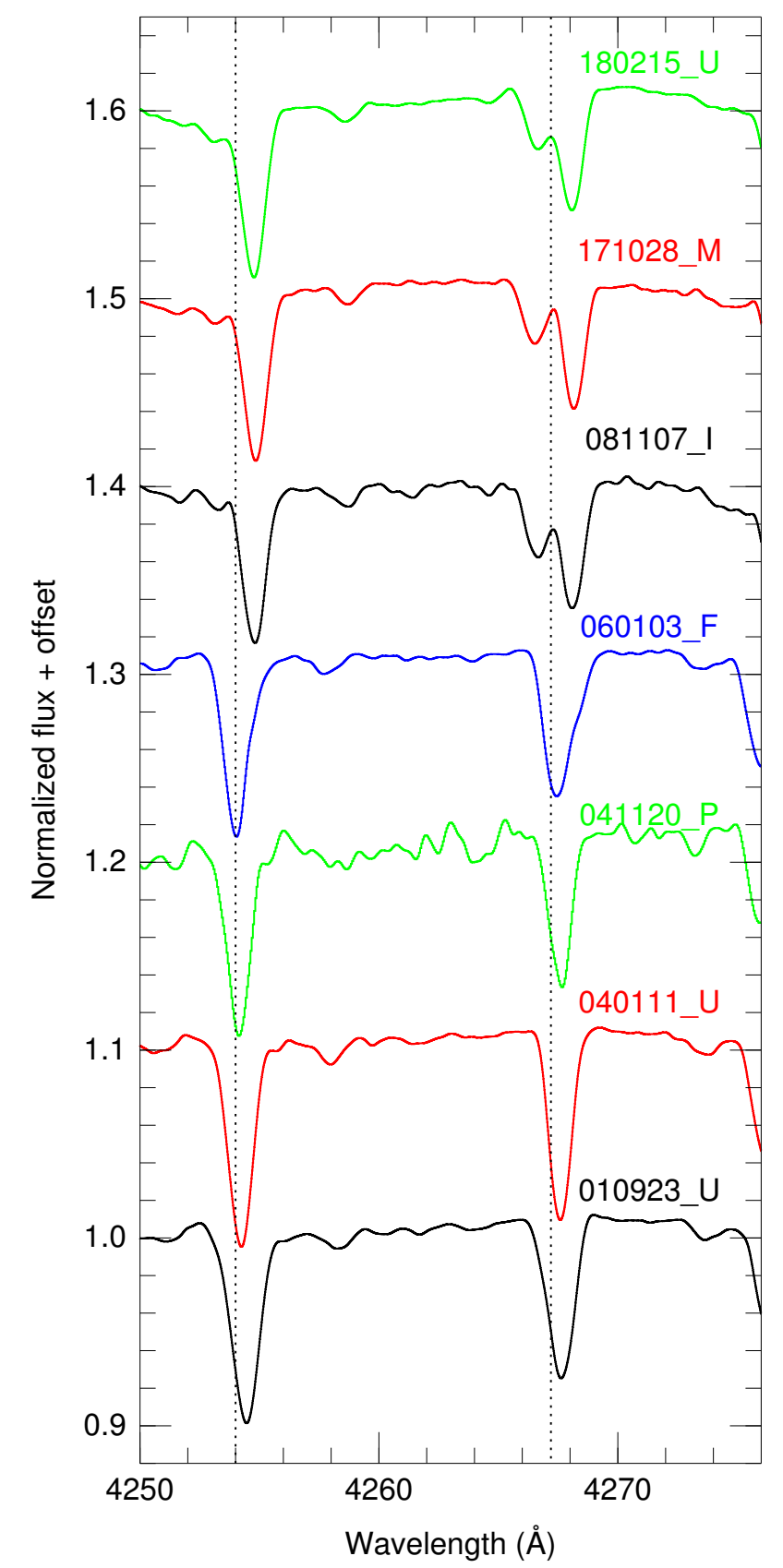

Fig. 3. Six LiLiMaRlin epochs of $\theta^{1}$ Ori D degraded to a common $R \sim$ 10000 . The two dotted lines indicate the rest wavelengths of O II $\lambda 4254$ and C II 24267 . The code for each spectra indicates the evening date as YYMMDD and the source (U, P, F, I, and M stand for UVES, OHP, FEROS, FIES, and Mercator, respectively).

amplitude does not agree with our measurements. If the double lines correspond to the two objects in the astrometric orbit determined by Karl et al. (2018), the semi-major axis and period are in the correct ballpark but the low inclination is incompatible with the high velocity amplitudes. Our mass ratio is $\sim 0.55$, which is not far from the Karl et al. (2018) value, and the deduced mass for the secondary (assuming their total mass) would be $9-10 M_{\odot}$, which is a reasonable value for a B2 V star. In any case, further data are needed to understand this system completely.

- $\theta^{1}$ Ori A (=HD 37020) is classified here as B0.2 V, which is changed from the previous one of B0.5 V by Simón-Díaz et al. (2006) based on the reorganization of 
spectral types in Sota et al. (2011) that moved $v$ Ori from B0 $\mathrm{V}$ to $09.7 \mathrm{~V}$ and $\tau$ Sco from B0.2 V to B0 V (B0.2 stars of luminosity class V now have He II $\lambda 4686 \sim$ Si III $\lambda 4552)$. The system includes two additional components (one astrometric and one eclipsing) that are too faint to contribute to the spectrogram (Karl et al. 2018). We also note that some references (e.g., Krełowski et al. 2016) claim that this star is of $\mathrm{O}$ type, which is usually caused by a confusion between the order in which components are named in most multiple systems (from bright to faint) and the way they are ordered in $\theta^{1}$ Ori (from west to east for A to D).

- $\theta^{1}$ Ori $\mathrm{Ba}, \mathrm{Bb}(=\mathrm{HD} 37021)$ is the faintest of the four systems in the Trapezium and the one with the most complex multiplicity, having at least six components (Karl et al. 2018). The subject of its (combined) spectral classification is complicated by the fact that the two primary components ( $\mathrm{Ba}$ and $\mathrm{Bb}$ in the WDS, $B_{1,5,6}$ and $B_{2,3}$ in Karl et al. 2018) are separated by 0.9 and their relative contribution to a spectrogram may depend on the setup and observing conditions. Nevertheless, that is not sufficient to explain the wide range of spectral classifications in the literature, which go from B1 V? (Slettebak 1963) to B6n (Trumpler 1931). Here we classify $\theta^{1}$ Ori $\mathrm{Ba}, \mathrm{Bb}$ as B3 Vn using either GOSSS or LiLiMaRlin, though the latter has significant nebular contamination. The classification is consistent with a high-order multiple system with a primary with $\sim 7 M_{\odot}$ and the $\Delta G \sim 1.5$ magnitude difference observed with respect to A or D.

- NU Ori (=HD 37061) is a quadruple system with three components ( $\mathrm{Aa}, \mathrm{Ab}$, and $\mathrm{C}$ ) that can be distinguished in high resolution, high $\mathrm{S} / \mathrm{N}$ spectra moving one relative to each other (Shultz et al. 2019). NU Ori C is interesting on its own as a magnetic star but the integrated light is dominated by Aa. Previous spectral classifications for the integrated spectrum are diverse but they concentrate around B0.5 V (with one claiming it is an O9 V star, Bragança et al. 2012). The GOSSS and LiLiMaRlin spectrograms clearly show that the He II lines are too strong for B0.5 (and way too weak for O9) and we classify it as B0 V(n), making it the third earliest-type system in Villafranca O-023, tied with $\theta^{1}$ Ori D.

- $\theta^{2}$ Ori B (=HD 37042) is the third brightest star in $G^{\prime}$ in the field, in part because of the higher extinction that affects the central part of the nebula (O'Dell et al. 2020). Simón-Díaz et al. (2006) classified it as B0.5 V. We find instead a slightly later type of $\mathrm{B} 0.7 \mathrm{~V}$ and we note how the $T_{\text {eff }}$ sequence in their Table 4 from $\theta^{1}$ Ori $\mathrm{D}(32 \mathrm{kK})$ to $\theta^{1}$ Ori A $(30 \mathrm{kK})$ to $\theta^{2}$ Ori B $(29 \mathrm{kK})$ corresponds in our spectral classifications to a sequence $\mathrm{B} 0 \mathrm{~V}$ to $\mathrm{B} 0.2 \mathrm{~V}$ to $\mathrm{B} 0.7 \mathrm{~V}$, which is the right direction. The spectral classification of $\theta^{2}$ Ori B as B2/5 by Hillenbrand (1997) is clearly discarded. - $\theta^{2}$ Ori C (=HD 37062) is classified here as B4 V. However, at the original LiLiMaRlin resolution, some spectra have double lines. The two components appear to have similar spectral types but different values of $v \sin i$ (one is a slow rotator and the other an intermediate one). The mass ratio must be close to one, as both components have a peak-to-peak amplitude of $\sim 60 \mathrm{~km} \mathrm{~s}^{-1}$. We thus confirm the spectroscopic binary nature detected by Corporon \& Lagrange (1999) but detecting two components instead of just one. Note the residual nebular emission lines characteristic of the low-excitation region of the Orion nebula where $\theta^{2}$ Ori $\mathrm{C}$ is located and which are difficult to eliminate in a faint star like this one.

The Gaia EDR3 source density is concentrated around the Trapezium and the parallax histogram of the whole field has a strong peak at the expected parallax dominated by sources with
$G^{\prime}$ in the 15-17 mag range. The membership algorithm, however, rejects most of those sources because the strong nebular contamination produces large $C^{*}$ values (see Fig. 4, changing the maximum $C^{*}$ to 3.0 , for example, increases the number of members from 114 to 158 while changing the cluster distance by just $0.6 \mathrm{pc}$ ). Therefore, the selected members are not centrally concentrated and are spread over most of the selected region. Assuming a spherical distribution, this effect should lead to a dispersion in distances of $\sim 0.7 \mathrm{pc}$, which is a factor of 3 lower than our uncertainty for the distance to Villafranca O-023 (see below). In addition, most of the bright stars in Villafranca O-023 have a bad RUWE and/or a large $\sigma_{\text {ext }}$ that causes their exclusion by the membership algorithm. The three brightest stars that are selected by the algorithm are $\theta^{1}$ Ori D, HD 36981, and HD 36982, with $\theta^{1}$ Ori $\mathrm{Ca}, \mathrm{Cb}$ and $\theta^{2}$ Ori $\mathrm{B}$ excluded only because of their large $\sigma_{\text {ext }}$. Three objects in Villafranca O-023, $\theta^{1}$ Ori D, HD 36982, and HD 36939, are among the eleven stars included in the second step of the membership selection process (see above).

There is a long history of distance measurements to objects in Villafranca O-023. The values up to 2008 are listed in Table 2 and plotted in Fig. 6 of Muench et al. (2008), who noted that "most measurements are accompanied by a large error bar, 15$20 \%$ " and that "there seems to be some emerging convergence at $400 \mathrm{pc}$ from the many varied techniques used in recent years". A more recent analysis with Gaia DR2 data (Kounkel et al. 2018) yields $389 \pm 3 \mathrm{pc}$ and is in excellent agreement with the $390 \pm 2 \mathrm{pc}$ value we derive here. Here we see a direct effect of the Gaia revolution. Thirteen years ago we were talking about distance uncertainties of 15-20\% and now we have two independent measurements that agree with uncertainties of less than $1 \%$.

The literature on runaway (and walkaway) stars from Villafranca O-023 reaches to the 1950s, as the prototype dynamical ejection that expelled $\mathrm{AE}$ Aur, $\mu \mathrm{Col}$, and $\iota$ Ori (Blaauw \& Morgan 1954; Hoogerwerf et al. 2000; Maíz Apellániz et al. 2018b) took place there $2.5 \mathrm{Ma}$ ago. Recent analyses have been carried out using Gaia DR2 (Schoettler et al. 2020; Farias et al. 2020) and HST (Platais et al. 2020) data. We have searched the Gaia EDR3 astrometry and found five walkaway/runaway candidates. Two of them, V1321 Ori and Brun 334, are listed as K stars in Skiff (2014) and were already identified as runaways by Schoettler et al. (2020) and Farias et al. (2020). For another two, BD -05 1322 (with different spectral classifications listed in Skiff 2014, some of them indicating broad lines) and HU Ori we have found no prior identifications as walkaways/runaways. In a separate paper (Maíz Apellániz et al. 2021d) we discuss the case of $\theta^{1}$ Ori F and we propose that it has been very recently ejected from the $\theta^{1}$ Ori $\mathrm{Ca}, \mathrm{Cb}$ system.

\subsubsection{Villafranca $\mathrm{O}-024=\gamma$ Velorum cluster $=$ Pozzo $1=$ Brandt 1}

This cluster was surprisingly not identified until two decades ago by Pozzo et al. (2000) and its discovery was prompted by the detection of the X-rays originating in its population of PMS stars. We say surprisingly because Villafranca O-024 includes $\gamma^{2} \mathrm{Vel}$, a WR+O system (WC8 + O7.5 V-III according to De Marco \& Schmutz 1999) in which the primary is the closest Wolf-Rayet and the only one ${ }^{3}$ within $1 \mathrm{kpc}$. Jeffries et al. (2009) studied the PMS stars in the cluster and determined their age at 5-10 Ma and Jeffries et al. (2014) identified two distinct kinematic populations, with the more likely scenario being that one corresponds to Villafranca O-024 (the cluster itself) and the

\footnotetext{
3 Using Gaia DR2 data, Rate \& Crowther (2020) gave a distance of $950 \pm 60$ pc for WR 94 but the Gaia EDR3 parallax places it slightly beyond $1 \mathrm{kpc}$.
} 


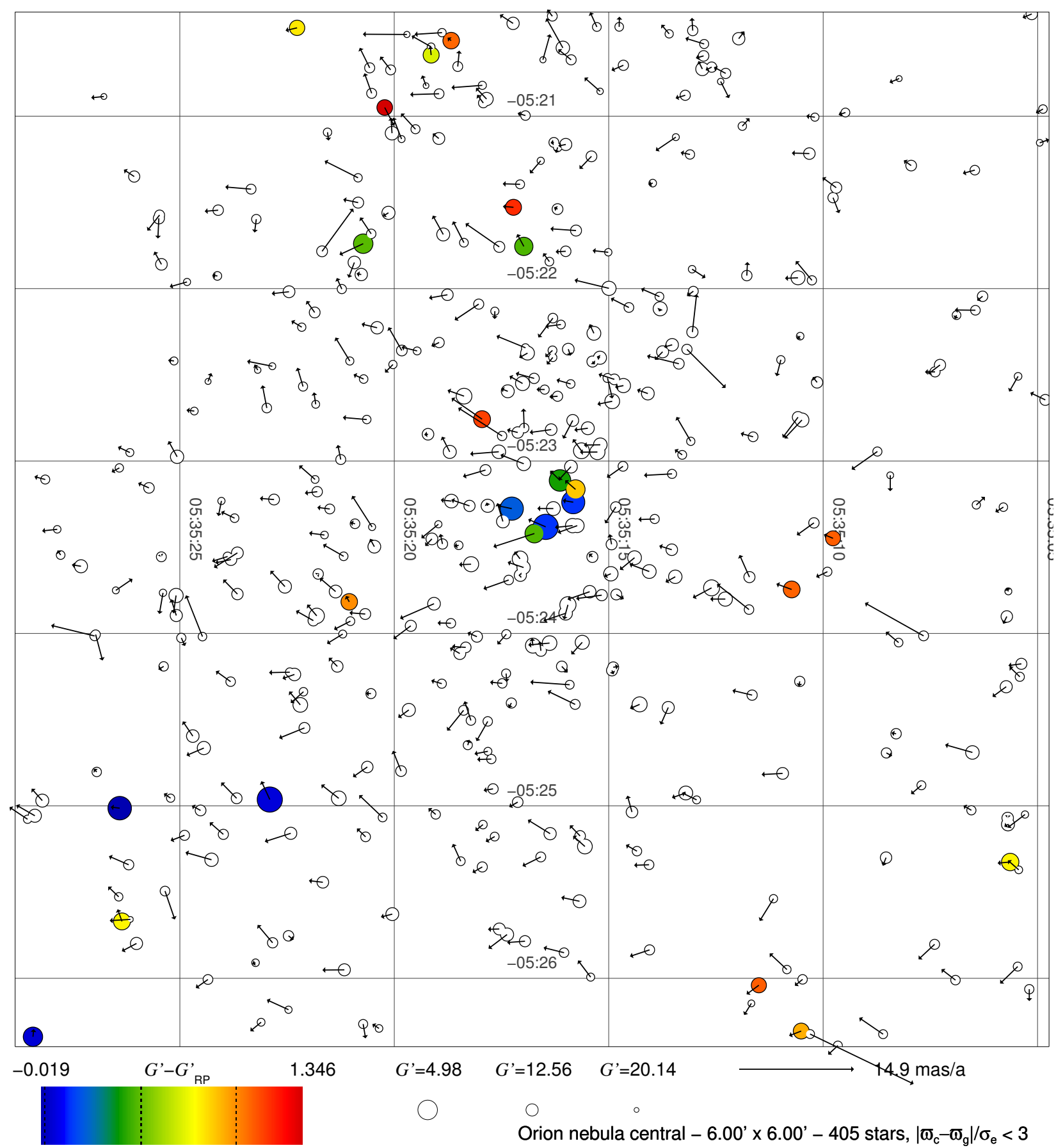

Fig. 4. Gaia EDR3 chart of the central $6^{\prime} \times 6^{\prime}$ of Villafranca O-023 (Orion nebula cluster). Plotted sources are those with normalized parallaxes within 3 sigmas independently of other filters i.e., a lax selection criterion for cluster membership rather than the strict one used to select the sample in this paper. Symbol color, symbol size, and arrows encode $G^{\prime}-G_{\mathrm{RP}}^{\prime}, G^{\prime}$, and proper motion, respectively, according to the caption. Objects without color either have no $G^{\prime}-G_{\mathrm{RP}}^{\prime}$ or (more commonly) have $C^{*}>0.4$ due to nebular contamination. The Trapezium is at the field center.

second one to the surrounding Vela OB2 association (Sacco et al. 2015). Prisinzano et al. (2016) determined that the low-mass stars follow a canonical IMF with a total of $100 M_{\odot}$ in the $0.16 M_{\odot}<M<1.3 M_{\odot}$ range.

We present GOSSS and LiLiMaRlin spectrograms of $\gamma^{2} \mathrm{Vel}$ in Figs. A.14 and A.23. The primary is classified as WC using GOSSS data and WC8 using LiLiMaRlin data, with the difference (as for WR 79) arising from the different wavelength ranges available. The secondary is classified as O7/8.5 III-II((f)) in both cases. We cannot provide a more detailed classification because we currently do not have a comparison WC8 spectrum and under such circumstances it is difficult to evaluate the contribution of each component (De Marco \& Schmutz 1999). We also present a GOSSS spectrogram of $\gamma^{1}$ Vel A,B in Fig. A.16. We classify it as B1.5 V, in agreement with Abt et al. (1976). We point out that Hernández \& Sahade (1980) identified $\gamma^{1}$ Vel A,B as an SB1 but there are no recent studies of this system and we have no LiLiMaRlin data. The WDS lists a visual component with a $\Delta m$ of 1.5 mag located at a separation of 37 mas, hence the A,B designation. 


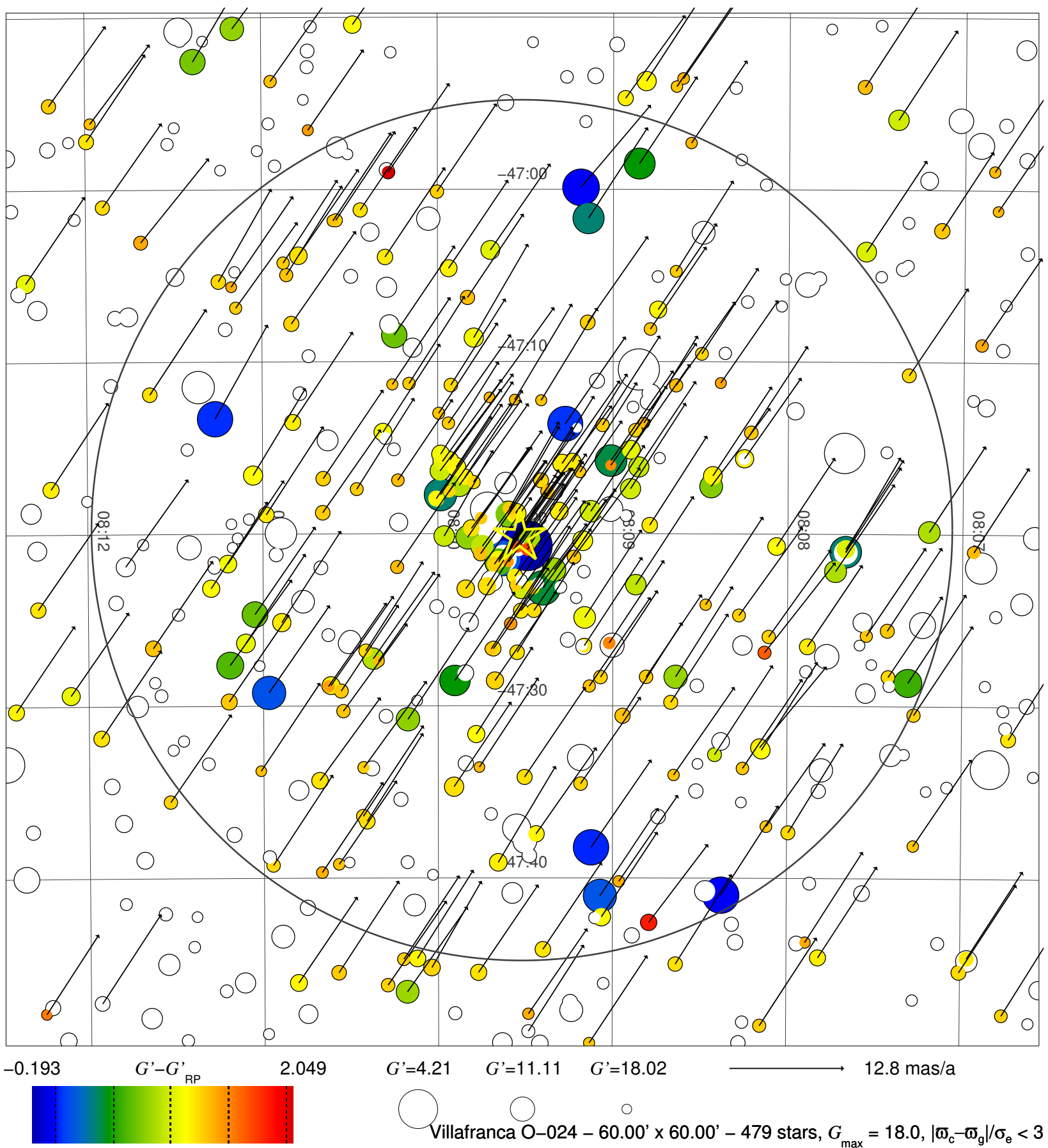

Fig. 5. Gaia EDR3 chart of the $1^{\circ} \times 1^{\circ}$ field centered on Villafranca O-024 ( $\gamma$ Vel cluster). Plotted sources are those with $G<18$ and with normalized parallaxes within 3 sigmas independently of other filters i.e., a lax selection criterion for cluster membership rather than the strict one used to select the sample in this paper. Symbol color, symbol size, and arrows encode $G^{\prime}-G_{\mathrm{RP}}^{\prime}, G^{\prime}$, and proper motion, respectively, according to the caption. Objects without color and arrows are those with a proper motion separation larger than $1.35 \mu$ as/a. The yellow star marks the location of $\gamma^{2}$ Vel (not a Gaia EDR3 source) and the large circle the circular aperture used to select cluster sources.

$\gamma^{2}$ Vel is so bright that it has no entry in Gaia EDR3. The second brightest star in Villafranca O-024 is $\gamma^{1}$ Vel A,B, which has a parallax and proper motion consistent with those of the cluster but its $\sigma_{\text {ext }}$ is 0.338 mas, so it is excluded by the selection algorithm. The next two bright stars with compatible distances are HD 68092 and HD 67820 but in both cases their proper motions are incompatible with that of the cluster (and do not point away from it, so they are not likely walkaways/runaways). The first stars selected by the algorithm are CPD $-462002 \mathrm{C}$ and D (note that A and B are $\gamma^{2}$ Vel and $\gamma^{1}$ Vel, respectively), HD 68157, and
HD 68009. An interesting feature of the Villafranca O-024 CMD in Fig. 1 is the presence of a nearly-equal-mass binary PMS located $\sim 0.75 \mathrm{mag}$ above the main one for $G_{\mathrm{BP}}^{\prime}-G_{\mathrm{RP}}^{\prime}$ between 2.2 and 3.5. We do not detect any runaways escaping from Villafranca O-024.

Given its proximity, Villafranca O-024 is the easiest cluster to detect in our sample with respect to the dominant background population, located at distances of $2-3 \mathrm{kpc}$, using proper motions (Fig. 5). The (mostly PMS) stars in the cluster are also relatively easy to distinguish in the CMD (Fig. 1), as already seen in Fig. 6 
of Jeffries et al. (2009). Indeed, the most likely contaminants in the sample would be from the B population (association members not in the cluster) of Jeffries et al. (2014). Stars with the proper motion of the cluster show a well defined core around $\gamma^{2}$ Vel and a halo while stars with other proper motions (many of them likely Vela OB2 members) are more uniformly scattered across the field.

Literature distances include measurements to $\gamma^{2} \mathrm{Vel}$ and to the cluster itself. Among the first, Millour et al. (2007) give $368_{-13}^{+38} \mathrm{pc}$ and North et al. (2007) $336_{-7}^{+8} \mathrm{pc}$ with interferometry while Maíz Apellániz et al. (2008) give $278_{-37}^{+50}$ pc with Hipparcos data using the van Leeuwen (2007) reduction and the same prior as here. As for cluster distances, Jeffries et al. (2009) used isochrone fitting to obtain $356 \pm 11 \mathrm{pc}$ and Franciosini et al. (2018) used Gaia DR2 parallaxes to obtain $345 \pm 12$ pc. Our new value of $336 \pm 1 \mathrm{pc}$ is much more precise but otherwise within 1.2 sigmas of the previous ones with the exception of that of Millour et al. (2007).

\subsubsection{Villafranca $\mathrm{O}-025=$ Trumpler $16 \mathrm{E}$}

The Carina nebula association (Car OB1) is the region with more $\mathrm{O}$ stars within $3 \mathrm{kpc}$ of the Sun. It is a complex region that was described in Villafranca I, where we analyzed Villafranca O-002 (Trumpler 14) and Villafranca O-003 (Trumpler $16 \mathrm{~W}$ ), see also the previous subsection. Trumpler 14 is a well defined cluster while Trumpler 16 is more extended and has no clear boundaries. In the previous paper we analyzed Trumpler 14 and the western part of Trumpler 16, as they are the two regions in Car OB 1 with the earliest-type $\mathrm{O}$ stars. Here we analyze the eastern part of Trumpler 16 (Trumpler 16 E or Villafranca O-025), which we define as the region around its most famous denizen, $\eta$ Car. $\eta$ Car is an eccentric binary system (Damineli et al. 2000, 2008; Iping et al. 2005) with an extremely luminous and massive LBV as a primary that has a historical record of extraordinary eruptions (Smith \& Frew 2011; Kiminki et al. 2016). There are at least twelve O-type systems ${ }^{4}$, of which the earliest ones are HDE 303308 A,B (O4.5 V((fc)), Sota et al. 2014) and CPD -59 2641 (O6 V((fc)), Sota et al. 2014).

We present GOSSS spectra of $\eta$ Car (Fig. A.15) and of four B-type stars: CPD -59 2598, CPD -59 2640, CPD -59 2616, and 2MASS J10452265-5942596 (Figs. A.16 and A.17). $\eta$ Car is classified simply as an LBV because the companion, whose spectrum has never been directly observed, leaves no trace in the spectrogram. We also present LiLiMaRlin spectra of four Otype SB2/SB3 binaries (V572 Car, CPD -59 2635, HD 93343, and V573 Car, Fig. A.22) that already had GOSSS classifications in either Sota et al. (2014) or Maíz Apellániz et al. (2016) but have been observed again at a more favorable phase for velocity separation. For V572 Car we show two epochs taken in consecutive nights of this interesting SB3 system, with the evening date encoded as YYMMDD in Fig. A.22. Two stars of spectral type $06.5 \mathrm{Vz}$ and B0 V orbit each other in $2.15 \mathrm{~d}$ (Rauw et al. 2001) and both trace a longer orbit around a B0.2 V star. Our classifications for the inner pair are slightly different from those of Rauw et al. (2001), of earlier type for the primary and of later type for the secondary. CPD -59 2635 is classified with LiLiMaRlin data as $\mathrm{O} 8 \mathrm{Vz}+\mathrm{O} 9.2 \mathrm{~V}$, which is similar but not identical to the GOSSS classification or to that of Albacete Colombo et al. (2001). For HD 93343, in

\footnotetext{
4 The census should be complete in the region barring the unknown classification of $\eta$ Car B and (unlikely) objects hidden by large extinction and source confusion (Smith 2006a; Preibisch et al. 2021).
}

Maíz Apellániz et al. (2016) we could only provide an O8 V combined classification, even though the presence of a secondary spectrum had already been detected by Walborn (1982) and has been classified as O8 + O8 by Rauw et al. (2009) and as O7.5 Vz + O7.5V (n)z by Putkuri et al. (2018). With the LiLiMaRlin spectrogram we classify it as $07.5 \mathrm{Vz}+07.5: \mathrm{V}(\mathrm{n})$, noting the uncertainty in the secondary caused by its moderately high rotation. V573 Car is classified with LiLiMaRlin data as O9.5 IV + B0.5 V, which is similar to the GOSSS classification but with narrower lines.

Not being a true cluster, Villafranca O-025 does not show a central concentration and the limits traditionally assigned to Trumpler 16 are established actually by the V-shaped dust lane immediately to its south, which is placed in the foreground with respect to the Carina nebula (top left plot in Fig. 7 of Maíz Apellániz \& Barbá 2018, see also Preibisch et al. 2021). Nevertheless, the stellar population of Villafranca O-025 is relatively easy to identify thanks to its richness and to its relatively low extinction in comparison with the background stars. Of the twelve $\mathrm{O}$ stars in Villafranca O-025, the membership algorithm selects nine, the six mentioned above plus CPD -59 2644, CPD -59 2627, and CPD -59 2629. The other three stars are excluded by their RUWE or lack of parallax: CPD -59 2636 A,B, CPD -59 2624, and CPD -59 2626 A,B. CPD -59 2635 is one of the eleven stars included in the second step of the membership selection process (see above). We do not detect any new runaways.

We measure a distance to Villafranca O-025 that is nearly identical to that of Villafranca O-003 and very similar to that of Villafranca O-002, When we also consider that the separation between Trumpler 14 and Trumpler 16 is large enough for the effects of the checkered pattern in the Gaia EDR3 zero point to be of the same order as the differences in parallax we observe, we conclude that the Gaia EDR3 results are compatible with all three groups being at the same distance. Differences of $50-100$ pc cannot be accurately detected for stellar groups at $2.0-2.5 \mathrm{kpc}$ with the current data. Nevertheless, it would be interesting to do a further study of Car OB1 with Gaia EDR3 surveying the whole association and trying to detect different kinematical components (see Damiani et al. 2017 for some hints of this). In this respect, we note that the proper motion of Villafranca O-025 is not identical to that of Villafranca O-003 and that the two groups we have defined in Trumpler 16 appear to be separating from each other.

\subsubsection{Villafranca $\mathrm{O}-026=\sigma$ Orionis cluster $=$ Escorial 7}

The $\sigma$ Orionis cluster is part of the Ori OB1d association (Caballero 2007; Bally 2008) and is the cluster with O-type stars (together with Villafranca O-024) with the lowest foreground extinction (Maíz Apellániz \& Barbá 2018). The natal cloud at the location of the cluster has already dispersed but its radiation reaches the nearby Horsehead nebula $\sim 30^{\prime}$ (3.5 pc) away and is the source of its formation and ionization. The central system of Villafranca O-026 is $\sigma$ Ori itself ${ }^{5}$, a high-order multiple whose most massive stars form a hierarchical triple whose nature remained hidden until recently. Traditionally, the system was composed of an optical pair A,B with a long period (at this point it has not completed one revolution since the first observed epoch) of 160 a but a decade ago Simón-Díaz et al. (2011) discovered that the A component is a spectroscopic binary with a

\footnotetext{
5 Following Caballero (2007), we recommend calling the cluster $\sigma$ Orionis and the stellar system $\sigma$ Ori.
} 
$143 \mathrm{~d}$ period and later Schaefer et al. (2016) resolved the pair with interferometry and determined a combined visual and spectroscopic orbit. In Maíz Apellániz et al. (2018a, 2019b, 2021c) we used lucky spectroscopy to separate (spatially or in velocity) the three components and derive spectral types of $\mathrm{O} 9.5 \mathrm{~V}$ for $\mathrm{Aa}$, $\mathrm{B} 0.2 \mathrm{~V}$ for $\mathrm{Ab}$, and B0.2 V(n) for B.

We present GOSSS and LiLiMaRlin spectrograms for three stars in Villafranca O-026 in Figs. A.17 and A.23. $\sigma$ Ori D is classified as B2 V(n) and we note that prior classifications did not include the (n) qualifier (it could be classified even as n). $\sigma$ Ori E is a well-known peculiar, magnetic, and He-rich B2 Vp star (Landstreet \& Borra 1978). HDE 294271 is also a moderately fast rotator classified as B5 V(n).

The combination of a very low extinction with a well defined PMS makes this cluster easy to separate from the other populations in the CMD. Surprisingly, Villafranca O-026 has very similar proper motions compared to the main background population despite the factor of $\sim 10$ in distance between the two, an effect likely caused by the proximity of the solar antapex. The brightest objects are not selected by the membership algorithm: $\sigma$ Ori Aa,Ab,B (one source in Gaia EDR3) has no parallax; $\sigma$ Ori E, $\sigma$ Ori D, HDE 294271 , HD 37525, and HDE 294272 B have $\sigma_{\text {ext }}>0.1$ (HDE 294271 also has RUWE =1.44). The brightest selected members are HD 37564 and HDE 294272 A. The cleaned CMD in Fig. 1 shows a large gap between the MS and the PMS starting at a main-sequence mass of $2 M_{\odot}$, a feature that is discussed below. We detect one possible runaway, Mayrit 968 292, that could have been ejected from the cluster core $\sim 330$ ka ago.

The value we derive for the distance to Villafranca O-026 of $397 \pm 2 \mathrm{pc}$ is in good agreement with the Gaia DR2 measurement of $403_{-7}^{+8} \mathrm{pc}$ of Zari et al. (2019) for their group $B_{2}$. However, it has a significant tension with the $387.5 \pm 1.5 \mathrm{pc}$ distance by Schaefer et al. (2016) from the orbital parallax of $\sigma$ Ori Aa,Ab, a $9.5 \mathrm{pc}$ difference that amounts to $2.4 \%$ or 3.8 sigmas. Given the cluster size (Caballero 2008), this cannot be a depth effect, so systematics must be present. One possible issue is the tension between the radial velocity curves derived by Simón-Díaz et al. (2015) and Schaefer et al. (2016) using only spectroscopic data: in Schaefer et al. (2016) $K_{\mathrm{Aa}}$ is smaller and $K_{\mathrm{Ab}}$ and $\gamma$ are larger than in Simón-Díaz et al. (2015). Such discrepancies when combining radial velocities from different instruments is, unfortunately, relatively common for $\mathrm{O}$ and earlyB stars (Trigueros Páez et al. 2021) and that could be the cause of the lower value for the orbital parallax, which is based on a combination of both.

\section{Analysis and future work}

\subsection{Comparison between Gaia DR2 and EDR3 distances}

As described in Maíz Apellániz et al. (2021e), the uncertainties derived from our method are a combination of random and systematic uncertainties, the latter being a consequence of the uncorrected parallax bias, $\sigma_{s}$. In the limit of a large number of sources located within a small region in the sky, Eq. (7) in that paper leads to a limit of $\sigma_{s}$ for the parallax uncertainty of the stellar group, $\sigma_{\varpi_{q}}$. That is indeed what we see in Table A.2 and its equivalents in Villafranca I (Table 3) and in Maíz Apellániz et al. (2021e) (Table 5), with a value of $43 \mu$ as for Gaia DR2 (Lindegren 2018) ${ }^{6}$ and of $10.3 \mu$ as for Gaia EDR3 (Maíz Apellániz et al. 2021e). The small deviations

\footnotetext{
6 The value is $21 \mu$ as for $G<13$, but most stars in a given group are
} fainter than that. from those values arise either because the number of stars is relatively small, in which case $\sigma_{\varpi_{g}}$ is larger (e.g., Villafranca O-013 and Villafranca O-014 SE in Table A.2) or because the group has a size large enough for the angular covariance effects to kick in, in which case $\sigma_{\varpi_{g}}$ is smaller (e.g., $\omega$ Cen or 47 Tuc in Maíz Apellániz et al. 2021e). Therefore, our group parallaxes (and the associated distances) are dominated by systematic uncertainties.

Going from parallaxes to distances implies the use of a prior and, in general, (assumed) Gaussian parallax uncertainties lead to asymmetric distance uncertainties (Maíz Apellániz 2005). Nevertheless, in the case of objects with small relative parallax uncertainties, $\sigma_{d} / d \approx \sigma_{\varpi} / \varpi$ is a good approximation and the asymmetry is small. For a constant parallax uncertainty (see above), this leads to the relative distance uncertainty being proportional to the distance, i.e.:

$\frac{\sigma_{d}}{d} \approx \sigma_{s} d$

where $\sigma_{s}$ is expressed in mas and $d$ is expressed in kpc. For Gaia EDR3, distances to the Villafranca groups have uncertainties around $1 \%$ at $1 \mathrm{kpc}$ and $3 \%$ at $3 \mathrm{kpc}$ while for Gaia DR2 the values are about four times higher. This approximation holds relatively well for the distances in Table A.2 and in Fig. 6. Comparing to the literature values in Villafranca I and in this paper leads us to conclude that the method in this paper with Gaia EDR3 values is the most precise to date to calculate distances to stellar groups in the solar neighborhood.

The calculated uncertainties using Gaia EDR3 are significantly lower than for Gaia DR2 but we should also ask ourselves if there is a systematic trend. Figure 6 indicates there is indeed such an effect, with most Gaia DR2 uncertainties being larger than their Gaia EDR3 counterparts, but it is not a large one. Taking the Gaia EDR3 values as the exact ones, the Gaia DR2 distances are, on average, 0.5 sigmas larger but none are larger than 1.2 sigmas. A single distance calculated from Gaia DR2 astrometry will be OK when judged by its uncertainty but a collection of them will be, on average, larger by $\sim 3 \%$ at $1 \mathrm{kpc}$ and by $\sim 8 \%$ at $3 \mathrm{kpc}$ with respect to Gaia EDR3 distances. This is likely a consequence of the improved calibration of Gaia EDR3: as for Gaia DR2 parallaxes a single quasar-based parallax zero point was used for all sources, it is possible that a small offset exists for DR2 parallaxes sources brighter than the average quasar. Such an effect would be compatible with the trend seen in Fig. 6 .

Another issue when validating a method to calculate distances is whether uncertainties are correctly estimated or not. In the case of the method here, we provide in Table A.2 for $t_{\varpi}$, the normalized $\chi^{2}$ test for the group parallax. The 26 values are between 0.78 and 1.22 and quite symmetrically distributed about the expected value of 1.00 , indicating that the individual parallaxes have correctly estimated uncertainties (see the discussion in the companion paper on the multiplicative constant $k$ ). Note that the equivalent $\chi^{2}$ tests for the proper motions are significantly larger than one in all cases. As discussed in Villafranca I, this is a consequence of the internal motions of the stellar groups and the values are larger when the targets are more massive or compact (larger physical velocities) and/or closer to us (larger proper motions due to the $1 / d$ factor).

History teaches us that most distance measurements in astronomy are plagued by systematic uncertainties and, indeed, that is also the case here. However, our analysis in Maíz Apellániz et al. (2021e) indicates that the systematic effects are well characterized, in part by correcting for them and in part by accounting for them in the error budget. Furthermore, 


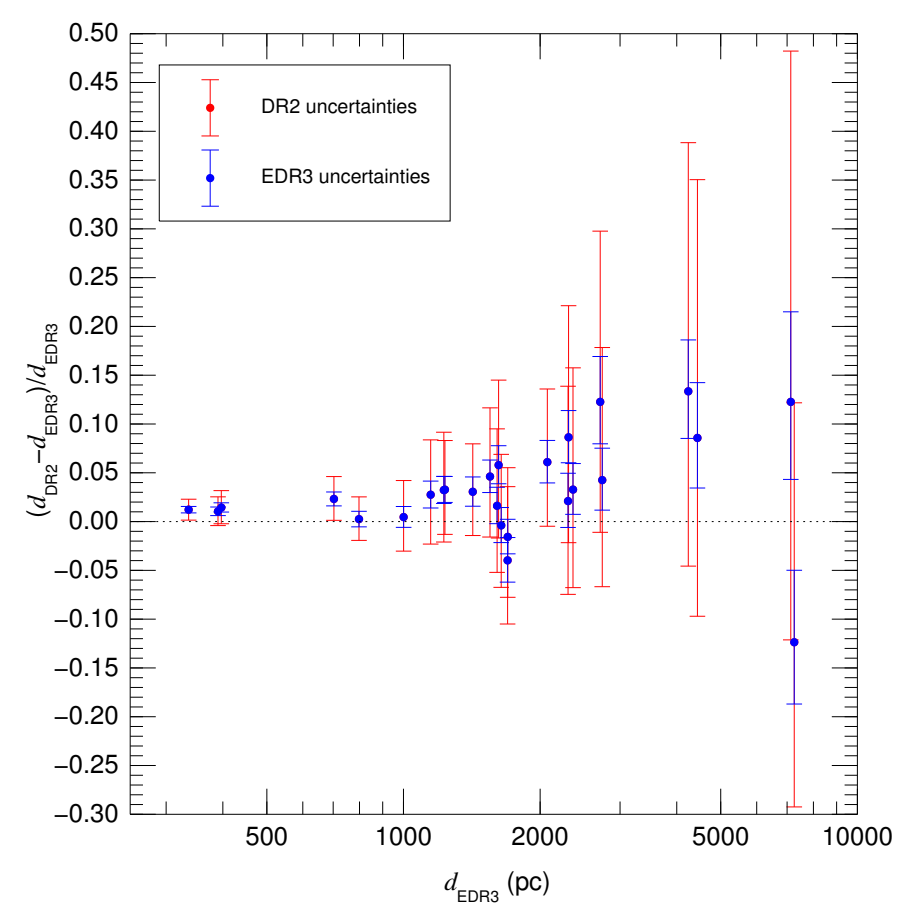

Fig. 6. Comparison between distances for the 26 Villafranca groups in this paper using Gaia DR2 and EDR3 data. The Gaia DR2 distances for the 16 groups in Villafranca I were recomputed to use the same selection parameters as in this paper. Red and blue are used to represent the Gaia DR2 and EDR3 uncertainties, respectively.

Table 3. Cluster members by mass range.

\begin{tabular}{lccc}
\hline \hline Cluster & $\begin{array}{c}2-50 M_{\odot} \\
\text { measured }\end{array}$ & $\begin{array}{c}8-50 M_{\odot} \\
\text { measured }\end{array}$ & $\begin{array}{c}8-50 M_{\odot} \\
\text { expected }\end{array}$ \\
\hline Villafranca O-026 & 11 & 3 & $1.7 \pm 1.2$ \\
Villafranca O-016 & 33 & 6 & $5.0 \pm 2.1$ \\
Villafranca O-021 & 44 & 5 & $6.7 \pm 2.4$ \\
Villafranca O-024 & 11 & 4 & $1.7 \pm 1.2$ \\
\hline
\end{tabular}

Notes. The last column is the expected result from a Kroupa IMF based on the measured number in the $2-50 M_{\odot}$ range.

the uncorrected systematic effects are of different sign in different parts of the sky, as they form a checkered pattern (Fig. 14 in Lindegren et al. 2021a), so, on average, distances to a large number of stellar groups scattered across the sky should still be unbiased. The ultimate answer to the possible presence of remaining systematic effects in the form of the distances in this paper being systematically higher or lower should come from a comparison with (yet inexistent) more accurate distances: only time will tell, as is often the case. However, we can still compare our results with the few high-accuracy geometric distances to some of the groups analyzed here and in previous papers: those of Thompson et al. (2020) for 47 Tuc (Maíz Apellániz et al. 2021e), Hensberge et al. (2000) for Villafranca O-020, North et al. (2007) for Villafranca O-024, and Smith (2006b) for $\eta$ Car in Villafranca O-025. The excellent agreement with those values in combination with the analysis in Maíz Apellániz et al. (2021e) leads us to conclude that our distances are accurate as systematic effects have been properly characterized.

\subsection{Orphan clusters}

One result of this series of papers is the observational demonstration of the existence of "orphan clusters", stellar groups in which the most massive stars have been ejected through a dynamical interaction and have left a system in which the present-day mass function (PDMF) is capped at a value significantly lower than that of the IMF (Oh et al. 2015). There are two clear examples in the current Villafranca sample: In Villafranca O-012 S (Haffner 18), HD 64568 (O3 V((f*))z) and HD $64315 \mathrm{~A}, \mathrm{~B}(\mathrm{O} 5.5 \mathrm{~V}+\mathrm{O} 7 \mathrm{~V})$ were ejected $\sim 400 \mathrm{ka}$ ago, leaving the cluster with two later O-type stars, CPD -26 2704 and CPD -26 2711. In Villafranca O-014 NW in the North America nebula, the two more massive objects, the Bajamar $(\mathrm{O} 3.5 \mathrm{III}(\mathrm{f} *)+\mathrm{O} 8$ :) and Toronto $(\mathrm{O} 6.5 \mathrm{~V}((\mathrm{f})) \mathrm{z}+\mathrm{B})$ stars were expelled in two events $1.5-1.6 \mathrm{Ma}$ ago as walkaways. Other OB stars were expelled in those two events and in another earlier one, leaving the cluster with apparently just two possible lateO-type stars (Maíz Apellániz et al. 2022). Both cases are similar in that one of the ejected systems contains a very-early-O star (hence, very massive) and the other one contains another $\mathrm{O}$ star earlier than O7. Another common characteristic is the complexity involved in the interactions that led to the ejections: in Villafranca O-014 NW both O-type systems are SB2s and were ejected in two different episodes that involved other stars and in Villafranca O-012 S HD 64315 A,B is a SB2+SB2 (Lorenzo et al. 2017) and HD 64568 appears to be single, implying a minimum of five stars.

Massive stars have been known to experience ejections from clusters by dynamical interactions since it was first proposed for AE Aur by Blaauw \& Morgan (1953). The novelty of orphan clusters lies in that the interaction takes place among the most massive stars in the system and is violent enough to unbind all of the participants. Are Villafranca O-012 S and Villafranca O014 NW unique in our sample? Apparently so but it is worth discussing two other related cases. In Villafranca O-004 (Westerlund 2) three very-early type stars, THA 35-II-42 (WR 21a), SS 215 (WR 20aa), and WR 20c appear to have been ejected but the cluster is significantly more massive and includes several other very massive stars (Villafranca I and references therein). The ejection of AE Aur, which also involved $\mu \mathrm{Col}$ and $\iota$ Ori, is interesting in that it involved five or six objects ${ }^{7}$ and in that it took place $2.5 \mathrm{Ma}$ ago (Hoogerwerf et al. 2000), significantly farther in the past than for Villafranca O-012 S or Villafranca O014 NW. Hoogerwerf et al. (2000) traced the three involved systems back to the same position where Villafranca O-023 was located at the time, suggesting that they were ejected from the Orion nebula cluster. As Villafranca O-023 currently contains $\theta^{1}$ Ori $\mathrm{Ca}, \mathrm{Cb}$, which is of earlier type than any of the ejected systems (of which $\iota$ Ori contains the star with the earliest spectral type, O8.5 III), this would not be truly an orphan cluster. However, the situation may be more complicated as the current Trapezium is younger than $2.5 \mathrm{Ma}\left(\theta^{1}\right.$ Ori $\mathrm{Ca}, \mathrm{Cb}$ should be located farther away from the ZAMS if it was of that age or older, the nebula itself is located very close to their ionizing sources, and the cluster itself is compact but in a hot dynamical state, de Grijs et al. 2008; Allison \& Goodwin 2011) and we are witnessing two generations of massive-star formation within Villafranca O-023: a first one that may have led to the ejection and

\footnotetext{
7 ८ Ori is a complex multiple system (Maíz Apellániz \& Barbá 2020) and it is unclear whether the B component is associated with the other three. At the time of Maíz Apellániz \& Barbá (2020), only Gaia DR2 was available and the A component had no parallax there. There is one available in Gaia EDR3 but its $\sigma_{\text {ext }}$ is too large to be useful.
} 
left an orphan cluster and a second one that produced the current Trapezium (Kroupa et al. 2018). If we also consider that the current 26 Villafranca groups are a small fraction of the known clusters with $\mathrm{O}$ stars in the Galaxy (which remain mostly unexplored with Gaia), cluster orphanization may be a significant phenomenon.

The existence of orphan clusters has important consequences in several fields of astrophysics. The most obvious one, as already mentioned, is that it introduces a large difference between the PDMF and the IMF, forcing a study of the proper motions and/or radial velocities in the cluster surroundings to detect the real maximal star mass (Weidner \& Kroupa 2006). The availability of Gaia astrometry is undoubtedly a game changer but any such study should consider the possibility of the walkaway or runaway stars having already exploded as $\mathrm{SNe}$. A related issue is the apparent age of the cluster as determined by the earliest-type star present among MS stars: if the most massive stars have already been ejected, we may think that the cluster is older than what it really is. We may also detect that the age as determined from PMS stars does not agree with the MS value. This effect is especially important when one considers that ejections take place preferentially right after cluster birth (Oh \& Kroupa 2016). The disappearance of the most massive stars from the core also reduces the ionization of the gas there and may favor the reignition of star formation there and the existence of multiple generations (Kroupa et al. 2018). Another effect is the spatial distribution of core-collapse $\mathrm{SNe}$ in the Galaxy, as an enhanced rate of massive-star ejections will scatter the explosions far from star formation regions (a star with an ejection velocity of $100 \mathrm{~km} \mathrm{~s}^{-1}$ travels $102 \mathrm{pc}$ in $1 \mathrm{Ma})$. Finally, the distribution and dynamics of compact objects is significantly altered: there would be fewer than expected in clusters and more in the diffuse population and some of the runaway neutron stars or black holes would be produced in binary systems previously ejected, leading to a double-runaway scenario (first by dynamical interaction and later by explosion) where the object is not moving away directly from any cluster (Pflamm-Altenburg \& Kroupa 2010).

\subsection{CMDs and group properties}

As previously discussed, the immediate goal in this paper of measuring distances to OB groups with precision and accuracy has been achieved. Of course, in the end we are interested in the properties of the groups themselves: masses, age, IMF, dynamical state, and extinction. Some of those properties can be obtained through their CMDs and they are the ultimate goal of the Villafranca project but also require additional information. In this subsection we discuss the challenges and present some first results. Regarding the challenges, there are several to consider:

- The first one is the one presented in the previous subsection. Clusters can eject OB stars and we have to search for possible runaways and walkaways.

- The selection criteria for cluster membership has to be less restrictive than the one used to obtain the distances. Regarding astrometry, our strategy when analyzing cluster properties will be to start with the known distance and make an initial cut based on normalized parallax of e.g., 3 sigmas and a larger $\sigma_{\text {ext }}$ than 0.1 mas. Stars with bad RUWE or with missing astrometry should be included on a one-by-one basis with the help of additional information.

- Gaia photometry (and future spectrophotometry) is compromised in crowded fields (e.g., Villafranca O-001) and nebular regions (e.g., Villafranca O-013 and Villafranca O-023).
In those cases it is useful to complement the bad-quality (as determined by $C^{*}$ ) or missing photometry with groundbased data. In our case, we plan to use photometry from GALANTE (Maíz Apellániz et al. 2021a) in future analyses of Galactic OB groups.

- Complementary studies using spectroscopy for massive stars and IR or X-ray for PMS stars are needed to constrain the properties. In the case of spectroscopy we will use the results from GOSSS in both hemispheres, from AstraLux (Maíz Apellániz 2010) and MONOS (Maíz Apellániz et al. 2019b; Trigueros Páez et al. 2021) in the northern hemisphere, and from OWN (Barbá et al. 2010, 2017) in the southern hemisphere to characterize the multiplicity of the O stars.

- In addition to binarity, massive stellar evolution can be strongly affected by rotation, to the point of rapid rotators evolving towards the left of the CMD after settling in the ZAMS (de Mink et al. 2010). Such rapid rotators have to be identified in order to correctly estimate their masses from a CMD.

- Many OB group studies assume that the extinction in front of the system is constant in both amount and type but, as shown by Maíz Apellániz \& Barbá (2018), important differences in both can be present and that is indeed what we see in some of the Villafranca groups. Gaia EDR3 in the form of a spread in the width of the isochrone. To tackle the issue, our future analyses in this project will use the Bayesian photometric tool CHORIZOS (Maíz Apellániz 2004) to calculate extinction on a star-by-star basis.

Given those challenges and that in some aspects we do not have all the necessary information at this point, here we present preliminary results just for the four nearby $\mathrm{OB}$ groups with the lowest extinction: Villafranca O-026 ( $\sigma$ Orionis cluster), Villafranca O-016 (NGC 2264), Villafranca O-021 (NGC 2362), and Villafranca O-024 ( $\gamma$ Velorum cluster). To select the sample in each cluster we used the previously obtained distances, eliminated the RUWE requirement, and included stars with $\sigma_{\text {ext }}<0.5$ mas, leaving the rest of the filters unchanged. The resulting CMDs are shown in Fig. 1, where we also included additional members and runaways/walkaways discussed in the text above. We have also plotted the Geneva MS isochrones (Lejeune \& Schaerer 2001), both without and with extinction, choosing the closest age (among 1.78 Ma, 3.16 Ma, and 10.0 Ma) to the one estimated for the cluster and the extinguished PMS isochrone from Baraffe et al. (2015) that is a best fit for the cluster $^{8}$. The photometry is not corrected from extinction but, given the low values measured (shown in Fig. 1 and taken from the data for a reference star from Maíz Apellániz \& Barbá 2018), its effect is small as evident from the two isochrones in each plot.

The observed CMDs (Fig. 1 are similar for the four clusters in showing a MS that extends from $\sim 2 M_{\odot}$ upwards and a well defined PMS that extends downwards. The PMS shows an equal-mass binary sequence $0.75 \mathrm{mag}$ above the primary one that is especially well visible for Villafranca O-024. The large dynamic range in magnitude provided by Gaia is at full display

\footnotetext{
8 The photometry for the MS isochrones is calculated from the SEDs using the synthetic photometry package used for CHORIZOS. Such SEDs are not available for the PMS isochrones, so we use their Gaia photometry downloaded from http://perso.ens-lyon.fr/ isabelle.baraffe/BHAC15dir/ and extinguished it using the values for a typical star. As a consequence, there may be small offsets at the level of a few hundredths of a magnitude which would correspond to age differences of $\sim 0.5 \mathrm{Ma}$. This would affect all clusters in a similar way, so the relative ages should not change.
} 
in Fig. 1. The first difference arises from the number of stars detected in each group, with Villafranca O-016 being the richest one and Villafranca O-026 the poorest one. A second difference is caused by the different distances: members are detected for all four clusters down to $G^{\prime} \sim 19.5 \mathrm{mag}$ (right vertical axes) but that value corresponds to different $G_{\text {abs }}$ (left vertical axes), allowing us to reach fainter absolute magnitudes for the two closest clusters, Villafranca O-024 and Villafranca O-026. The spread in $G_{\mathrm{BP}}^{\prime}-G_{\mathrm{RP}}^{\prime}$ for faint stars is mostly caused by large uncertainties in the measured colors. The third and most relevant difference is the separation between the MS (as extrapolated from that for massive stars) and the observed PMS. To determine it, we have measured the $\Delta\left(G_{\mathrm{BP}}^{\prime}-G_{\mathrm{RP}}^{\prime}\right)$ at the position expected for a $1 M_{\odot}$ at the MS and marked it with orange lines in Fig. 1. The measured values determine an age sequence from the youngest, Villafranca O-026 (0.7 mag), to Villafranca O-016 (0.6 mag) to Villafranca O-021 (0.5 mag) to the oldest, Villafranca O-024 (0.4 mag), as the PMS stars approach the MS as they age. The corresponding ages from fitting the best PMS isochrone from Baraffe et al. (2015) are $2.0 \pm 0.5 \mathrm{Ma}, 4 \pm 2 \mathrm{Ma}, 5.0 \pm 0.5 \mathrm{Ma}$, and $8 \pm 2 \mathrm{Ma}$, respectively, with an estimated additional systematic uncertainty of $0.5 \mathrm{Ma}$ due to the photometric calibration (see footnote 8). Those ages are in good agreement with the values (a) for Villafranca O-026 from Zapatero Osorio et al. (2002) of 2-4 Ma based on Li I $\lambda 6708$ absorption, (b) for Villafranca O-016 from Sung et al. (2004) of 3.1 \pm 0.5 Ma based on PMS fitting, (c) for Villafranca O-021 from Dahm (2005) of 3.55.0 Ma based on PMS fitting, and (d) for Villafranca O-024 from Jeffries et al. (2009) of 5-10 Ma from a combination of methods. The large spread of the PMS (and corresponding uncertainty in its age) for Villafranca O-016 may be caused by differential extinction, known to exist in NGC 2264, or by a mixture of ages.

We observe no significant discrepancies between the PMS ages and the ones constrained by the upper main sequence. However, the latter age constraints are weak (assuming low-rotation, single-star-like evolution), given that these clusters are relatively low mass and only have one or two O stars each. Villafranca O026 only has one late-O dwarf, so that only gives an upper age limit of $\sim 5 \mathrm{Ma}$. The earliest O-type in Villafranca O-016 is an $\mathrm{O} 7 \mathrm{~V}$, constraining the age to be $4 \mathrm{Ma}$ or less. Villafranca O-021 contains two evolved late-O stars, giving an upper age limit of 6$7 \mathrm{Ma}$. Finally, the most massive system in Villafranca O-024 is composed of an evolved WR $+\mathrm{O}$ binary, whose components are unlikely to have followed a single-star-like evolution, in which case they can be somewhat older than an isolated $\mathrm{O}$ star.

The single most interesting CMD in Fig. 1 is that of Villafranca O-026, the youngest and less massive of the four. The only two objects found between the end of the PMS isochrone (at a mass of $\sim 1.4 M_{\odot}$ ) and the first objects close to the MS isochrone (at a mass of $\sim 2.0 M_{\odot}$ ) had to be inserted by hand as one of them (Mayrit 240322) has poor-quality astrometry and the other one (Mayrit 968292) is outside the region but is a possible runaway. The gap is very prominent in the CMD: if we exclude the two doubtful stars, it amounts to $1.2 \mathrm{mag}$ in $G_{\mathrm{BP}}^{\prime}-G_{\mathrm{RP}}^{\prime}$ and $2.4 \mathrm{mag}$ in $G_{\mathrm{abs}}$. This could indicate that stars with $\gtrsim 2 M_{\odot}$ evolve towards the MS significantly faster than lower-mass objects, an effect already apparent in Fig. 14 of Peña Ramírez et al. (2012). It would be interesting to test this hypothesis with other clusters of similar age, especially if they are more massive and better populated.

We finally make an estimate of the IMF for the upper MS of each of the four clusters by using the CMDs to count the number of stars with masses in the $8-50 M_{\odot}$ range (massive stars) and in the $2-50 M_{\odot}$ range (intermediate and massive stars), for which a Kroupa IMF predicts a ratio of 0.15 . Known binary stars are separated to be properly accounted for and the results are listed in Table 3. For the two most massive clusters (Villafranca O016 and Villafranca O-021) the measured number of stars in the $8-50 M_{\odot}$ range is within one sigma of the expected value from a Kroupa IMF. The two less massive clusters (Villafranca O026 and Villafranca O-024) have 3 and 4 stars, respectively, in the $8-50 M_{\odot}$ range, which in both cases implies a slight overrepresentation of massive stars (between one and two sigmas) with respect to a Kroupa IMF and is a likely manifestation of the increased stochasticity of the IMF for small clusters. In the case of the two older clusters it is possible that some stars have already exploded as $\mathrm{SNe}$, which if true would be especially relevant for Villafranca O-024, as that would push its number of initial $8-50 M_{\odot}$ stars beyond a two-sigma result for a Kroupa IMF. For this cluster Prisinzano et al. (2016) measured a total mass of $100 M_{\odot}$ in the $0.16-1.30 M_{\odot}$ range distributed in a manner consistent with a Kroupa IMF there. An extrapolation into larger masses from those values assuming a Kroupa IMF yields 15.5 stars in the $2-50 M_{\odot}$ range and 2.4 stars in the $8-50 M_{\odot}$ range. Both results are approximately one sigma from the measured numbers of 11 and 4 but in opposite directions, indicating that the cluster is slightly deficient in intermediate-mass stars and slightly overabundant in massive ones.

\subsection{Future work}

Our immediate plans are to include more OB groups in the Villafranca sample using Gaia EDR3, GOSSS, and LiLiMaRlin data. The project will be coordinated with the ALS catalog (Reed 2003; Pantaleoni González et al. 2021), which is compiling and analyzing the OB stars included in Gaia. The next Villafranca paper will concentrate on the solar neighborhood to complement the analysis of Pantaleoni González et al. (2021) on the mapping of the spatial distribution of massive stars and will also include clusters with B but no O stars. In the long term, Gaia DR4 data will be incorporated into our analysis.

Acknowledgements. We thank J. A. Caballero for his help with the Mayrit stars of Escorial 7. J.M.A., R.F.A., M.P.G., P.C.B., and A.S. acknowledge support from the Spanish Government Ministerio de Ciencia e Innovación through grant PGC2018-095 049-B-C22. R. H. B. acknowledges support from ANID FONDECYT Regular Project 1211903 and the ESAC visitors program. E.J.A. acknowledges support from the State Agency for Research of the Spanish Government Ministerio de Ciencia e Innovación through the "Center of Excellence Severo Ochoa" award to the Instituto de Astrofísica de Andalucía (SEV-2017-0709) and through grant PGC2018-095 049-B-C21 This work has made use of data from the European Space Agency (ESA) mission Gaia, processed by the Gaia Data Processing and Analysis Consortium (DPAC). Funding for the DPAC has been provided by national institutions, in particular the institutions participating in the Gaia Multilateral Agreement. The Gaia data is processed with the computer resources at Mare Nostrum and the technical support provided by BSC-CNS. This research has made extensive use of the SIMBAD and VizieR databases, operated at CDS, Strasbourg, France.

\section{References}

Abt, H. A., Landolt, A. U., Levy, S. G., \& Mochnacki, S. 1976, AJ, 81, 541 Aidelman, Y., Cidale, L. S., Zorec, J., \& Panei, J. A. 2018, A\&A, 610, A30 Albacete Colombo, J. F., Morrell, N. I., Niemelä, V. S., \& Corcoran, M. F. 2001, MNRAS, 326, 78

Allison, R. J., \& Goodwin, S. P. 2011, MNRAS, 415, 1967

Arias, J. I., Morrell, N. I., Barbá, R. H., et al. 2002, MNRAS, 333, 202

Arias, J. I., Barbá, R. H., Maíz Apellániz, J., Morrell, N. I., \& Rubio, M. 2006, MNRAS, 366, 739

Arias, J. I., Barbá, R. H., Gamen, R. C., et al. 2010, ApJ, 710, L30

Arias, J. I., Walborn, N. R., Simón Díaz, S., et al. 2016, AJ, 152, 31 
Bally, J. 2008, in Overview of the Orion Complex, ed. B. Reipurth, ASP, 4, 459 Baraffe, I., Homeier, D., Allard, F., \& Chabrier, G. 2015, A\&A, 577, A42 Barbá, R. H., \& Arias, J. I. 2007, A\&A, 471, 841

Barbá, R. H., Gamen, R. C., Arias, J. I., et al. 2010, RMxAC, 38, 30

Barbá, R. H., Gamen, R., Arias, J. I., \& Morrell, N. I. 2017, in The Lives and Death-Throes of Massive Stars, IAU Symp., 329, 89

Blaauw, A., \& Morgan, W. W. 1953, Bull. Astron. Inst. Netherlands, 12, 76

Blaauw, A., \& Morgan, W. W. 1954, ApJ, 119, 625

Bragança, G. A., Daflon, S., Cunha, K., et al. 2012, AJ, 144, 130

Brown, A. G. A., Vallenari, A., Prusti, T., et al. 2021, A\&A, 649, A1

Caballero, J. A. 2007, A\&A, 466, 917

Caballero, J. A. 2008, MNRAS, 383, 375

Campillay, A. R., Arias, J. I., Barbá, R. H., et al. 2019, MNRAS, 484, 2137

Cantat-Gaudin, T., \& Brandt, T. D. 2021, A\&A, 649, A124

Cantat-Gaudin, T., Jordi, C., Vallenari, A., et al. 2018, A\&A, 618, A93

Carpenter, J. M., Heyer, M. H., \& Snell, R. L. 2000, ApJS, 130, 381

Corporon, P., \& Lagrange, A. M. 1999, A\&AS, 136, 429

Crampton, D., Georgelin, Y. M., \& Georgelin, Y. P. 1978, A\&A, 66, 1

Crowther, P. A., De Marco, O., \& Barlow, M. J. 1998, MNRAS, 296, 367

Dahm, S. E. 2005, AJ, 130, 1805

Dahm, S. E. 2008, in NGC 2362: The Terminus of Star Formation, ed. B. Reipurth, ASP, 5, 26

Damineli, A., Kaufer, A., Wolf, B., et al. 2000, ApJ, 528, L101

Damineli, A., Hillier, D. J., Corcoran, M. F., et al. 2008, MNRAS, 384, 1649

Damiani, F., Klutsch, A., Jeffries, R. D., et al. 2017, A\&A, 603, A81

de Grijs, R., Goodwin, S. P., Kouwenhoven, M. B. N., \& Kroupa, P. 2008, A\&A 492,685

De Marco, O., \& Schmutz, W. 1999, A\&A, 345, 163

de Mink, S. E., Cantiello, M., Langer, N., \& Pols, O. R. 2010, in American Institute of Physics Conference Series, ed. V. Kologera, \& M. van der Sluys, 1314,291

Evans, C. J., Smartt, S. J., Lee, J.-K., et al. 2005, A\&A, 437, 467

Fabry, M., Hawcroft, C., Frost, A. J., et al. 2021, A\&A, 651, A119

Farias, J. P., Tan, J. C., \& Eyer, L. 2020, ApJ, 900, 14

Feast, M. W., Thackeray, A. D., \& Wesselink, A. J. 1955, MNRAS, 67, 51

Ferrero, G., Gamen, R., Benvenuto, O., \& Fernández-Lajús, E. 2013, MNRAS, 433,1300

Foster, T., \& MacWilliams, J. 2006, ApJ, 644, 214

Franciosini, E., Sacco, G. G., Jeffries, R. D., et al. 2018, A\&A, 616, L12

Garmany, C. D., \& Stencel, R. E. 1992, A\&AS, 94, 211

Hensberge, H., Pavlovski, K., \& Verschueren, W. 2000, A\&A, 358, 553

Hernández, C. A., \& Sahade, J. 1980, PASP, 92, 819

Hester, J. J., Scowen, P. A., Sankrit, R., et al. 1996, AJ, 111, 2349

Hillenbrand, L. A. 1997, AJ, 113, 1733

Hillenbrand, L. A., Massey, P., Strom, S. E., \& Merrill, K. M. 1993, AJ, 106, 1906

Hiltner, W. A., \& Iriarte, B. 1955, ApJ, 122, 185

Hoffmeister, V. H., Chini, R., Scheyda, C. M., et al. 2008, ApJ, 686, 310

Hoogerwerf, R., de Bruijne, J. H. J., \& de Zeeuw, P. T. 2000, ApJ, 544, 133L

Humphreys, R. M. 1978, ApJS, 38, 309

Iping, R. C., Sonneborn, G., Gull, T. R., Massa, D. L., \& Hillier, D. J. 2005, ApJ, 633, L37

Ishida, K. 1970, PASJ, 22, 277

Jeffries, R. D., Naylor, T., Walter, F. M., Pozzo, M. P., \& Devey, C. R. 2009, MNRAS, 393, 538

Jeffries, R. D., Jackson, R. J., Cottaar, M., et al. 2014, A\&A, 563, A94

Johnson, H. L., \& Morgan, W. W. 1953, ApJ, 117, 313

Karl, M., Pfuhl, O., Eisenhauer, F., et al. 2018, A\&A, 620, A116

Kharchenko, N. V., Piskunov, A. E., Schilbach, E., Röser, S., \& Scholz, R. D 2012, A\&A, 543, A156

Kiminki, D. C., Kobulnicky, H. A., Kinemuchi, K., et al. 2007, ApJ, 664, 1102

Kiminki, M. M., Reiter, M., \& Smith, N. 2016, MNRAS, 463, 845

Kounkel, M., Covey, K., Suárez, G., et al. 2018, AJ, 156, 84

Kraus, S., Weigelt, G., Balega, Y. Y., et al. 2009, A\&A, 497, 195

Krełowski, J., Galazutdinov, G. A., Strobel, A., \& Mulas, G. 2016, Acta Astron., 66,469

Kroupa, P., Jeřábková, T., Dinnbier, F., Beccari, G., \& Yan, Z. 2018, A\&A, 612, A74

Kuhn, M. A., \& Hillenbrand, L. A. 2020, Res. Notes Am. Astron. Soc., 4, 224

Kuhn, M. A., Hillenbrand, L. A., Sills, A., Feigelson, E. D., \& Getman, K. V. 2019, ApJ, 870, 32

Kuhn, M. A., Hillenbrand, L. A., Carpenter, J. M., \& Avelar Menéndez, Á. R. 2020, ApJ, 899, 128

Landstreet, J. D., \& Borra, E. F. 1978, ApJ, 224, L5

Lejeune, T., \& Schaerer, D. 2001, A\&A, 366, 538

Levenhagen, R. S., \& Leister, N. V. 2006, MNRAS, 371, 252

Lindegren, L., Klioner, S. A., Hernández, J., et al. 2021a, A\&A, 649, A2

Lindegren, L., Bastian, U., Biermann, M., et al. 2021b, A\&A, 649, A4
Lindegren, L., Hernández, J., Bombrun, A., et al. 2018, http: //www. cosmos.esa.int/documents/29201/1770596/Lindegren_ GaiaDR2_Astrometry_extended.pdf

Linder, N., Rauw, G., Sana, H., De Becker, M., \& Gosset, E. 2007, A\&A, 474, 193

Lodén, L. O. 1966, Ark. Astron., 4, 65

Lorenzo, J., Simón-Díaz, S., Negueruela, I., et al. 2017, A\&A, 606, A54

Luri, X., Chemin, L., Clementini, G., et al. 2021, A\&A, 649, A7

MacKenty, J. W., Maíz Apellániz, J., Pickens, C. E., Norman, C. A., \& Walborn, N. R. 2000, AJ, 120, 3007

Maíz Apellániz, J. 2001, AJ, 121, 2737

Maíz Apellániz, J. 2004, PASP, 116, 859

Maíz Apellániz, J. 2005, in The Three-Dimensional Universe with Gaia, eds. C. Turon, K. S. O'Flaherty, \& M. A. C. Perryman, ESA SP, 576, 179

Maíz Apellániz, J. 2010, A\&A, 518, A1

Maíz Apellániz, J. 2019, A\&A, 630, A119 (Villafranca 0)

Maíz Apellániz, J. 2022, A\&A, 657, A130

Maíz Apellániz, J., \& Barbá, R. H. 2018, A\&A, 613, A9

Maíz Apellániz, J., \& Weiler, M. 2018, A\&A, 619, A180

Maíz Apellániz, J., \& Barbá, R. H. 2020, A\&A, 636, A28

Maíz Apellániz, J., Pérez, E., \& Mas-Hesse, J. M. 2004, AJ, 128, 1196

Maíz Apellániz, J., Alfaro, E. J., \& Sota, A. 2008, ArXiv e-prints [arXiv:0804.2553]

Maíz Apellániz, J., Sota, A., Walborn, N. R., et al. 2011, HSA, 6, 467

Maíz Apellániz, J., Pellerin, A., Barbá, R. H., et al. 2012, in Astronomical Society of the Pacific Conference Series, eds. L. Drissen, C. Robert, N. StLouis, \& A. F. J. Moffat, 465, 484

Maíz Apellániz, J., Alfaro, E. J., Arias, J. I., et al. 2015a, HSA, 8, 603

Maíz Apellániz, J., Negueruela, I., Barbá, R. H., et al. 2015b, A\&A, 579, A108

Maíz Apellániz, J., Úbeda, L., Barbá, R.-H., et al. 2015c, HSA, 8, 604

Maíz Apellániz, J., Sota, A., Arias, J. I., et al. 2016, ApJS, 224, 4

Maíz Apellániz, J., Barbá, R. H., Simón-Díaz, S., et al. 2018a, A\&A, 615, A161

Maíz Apellániz, J., Pantaleoni González, M., Barbá, R. H., et al. 2018b, A\&A, 616, A149

Maíz Apellániz, J., Trigueros Páez, E., Jiménez Martínez, I., et al. 2019a, HSA, 10, 420 (LiLiMaRlin)

Maíz Apellániz, J., Trigueros Páez, E., Negueruela, I., et al. 2019b, A\&A, 626, A20

Maíz Apellániz, J., Crespo Bellido, P., Barbá, R.H., Fernández Aranda, R., \& Sota, A. 2020, A\&A, 643, A138 (Villafranca I)

Maíz Apellániz, J., Alfaro, E. J., Barbá, R. H., et al. 2021a, MNRAS, 506, 3138

Maíz Apellániz, J., Barbá, R. H., Caballero, J. A., Bohlin, R. C., \& Fariña, C. 2021b, MNRAS, 501, 2487

Maíz Apellániz, J., Barbá, R. H., Fariña, C., et al. 2021c, A\&A, 646, A11

Maíz Apellániz, J., Pantaleoni González, M., \& Barbá, R.H. 2021d, Res. Notes Am. Astron. Soc., 5, 232

Maíz Apellániz, J., Pantaleoni González, M., \& Barbá, R. H. 2021e, A\&A, 649, A13

Maíz Apellániz, J., Pantaleoni González, M., Barbá, R.H., \& Weiler, M. 2022, A\&A, 657, A72

Marco, A., \& Negueruela, I. 2017, MNRAS, 465, 784

Mason, B. D., Wycoff, G. L., Hartkopf, W. I., Douglass, G. G., \& Worley, C. E. 2001, AJ, 122, 3466

Massey, P., Johnson, K. E., \& Degioia-Eastwood, K. 1995, ApJ, 454, 151

Megeath, S. T., Townsley, L. K., \& Oey, M. S. 2008, in Low and High Mass Star Formation in the W3, W4, and W5 Regions, ed. B. Reipurth, ASP, 4, 264

Millour, F., Petrov, R. G., Chesneau, O., et al. 2007, A\&A, 464, 107

Muench, A., Getman, K., Hillenbrand, L., \& Preibisch, T. 2008, in Star Formation in the Orion Nebula I: Stellar Content, ed. B. Reipurth, ASP, 4, 483

Mužić, K., Scholz, A., Peña Ramírez, K., et al. 2019, ApJ, 881, 79

North, J. R., Tuthill, P. G., Tango, W. J., \& Davis, J. 2007, MNRAS, 377, 415

O'Dell, C. R., Muench, A., Smith, N., \& Zapata, L. 2008, in Star Formation in the Orion Nebula II: Gas, Dust, Proplyds and Outflows, ed. B. Reipurth, ASP, 4,544

O’Dell, C. R., Kollatschny, W., \& Ferland, G. J. 2017, ApJ, 837, 151

O’Dell, C. R., Abel, N. P., \& Ferland, G. J. 2020, ApJ, 891, 46

Oh, S., \& Kroupa, P. 2016, A\&A, 590, A107

Oh, S., Kroupa, P., \& Pflamm-Altenburg, J. 2015, ApJ, 805, 92

Oliveira, J. M. 2008, in Star Formation in the Eagle Nebula, ed. B. Reipurth, ASP, 5, 599

Peña Ramírez, K., Béjar, V. J. S., Zapatero Osorio, M. R., Petr-Gotzens, M. G., \& Martín, E. L. 2012, ApJ, 754, 30

Pantaleoni González, M., Maíz Apellániz, J., Barbá, R. H., \& Reed, B. C. 2021, MNRAS, 504, 2968

Pflamm-Altenburg, J., \& Kroupa, P. 2010, MNRAS, 404, 1564

Platais, I., Robberto, M., Bellini, A., et al. 2020, AJ, 159, 272 
Pozzo, M., Jeffries, R. D., Naylor, T., et al. 2000, MNRAS, 313, L23

Preibisch, T., Flaischlen, S., Göppl, C., Ercolano, B., \& Roccatagliata, V. 2021, A\&A, 648, A34

Prisinzano, L., Damiani, F., Micela, G., et al. 2016, A\&A, 589, A70

Prusti, T., de Bruijne, J. H. J., Brown, A. G. A., et al. 2016, A\&A, 595, A1

Putkuri, C., Gamen, R., Morrell, N. I., et al. 2018, A\&A, 618, A174

Rate, G., \& Crowther, P. A. 2020, MNRAS, 493, 1512

Rauw, G., Sana, H., Antokhin, I. I., et al. 2001, MNRAS, 326, 1149

Rauw, G., Nazé, Y., Fernández Lajús, E., et al. 2009, MNRAS, 398, 1582

Rauw, G., Sana, H., Spano, M., et al. 2012, A\&A, 542, A95

Reed, B. C. 2003, AJ, 125, 2531

Reipurth, B. 2008, in Young Stars in NGC 6231 and the Sco OB1 Association, ed. B. Reipurth, ASP, 5, 401

Renzo, M., Zapartas, E., de Mink, S. E., et al. 2019, A\&A, 624, A66

Riello, M., De Angeli, F., Evans, D. W., et al. 2021, A\&A, 649, A3

Román-Zúñiga, C. G., \& Lada, E. A. 2008, in Star Formation in the Rosette Complex, ed. B. Reipurth, ASP, 4, 928

Rosslowe, C. K., \& Crowther, P. A. 2015, MNRAS, 447, 2322

Sacco, G. G., Jeffries, R. D., Randich, S., et al. 2015, A\&A, 574, L7

Sana, H., Rauw, G., \& Gosset, E. 2007, ApJ, 659, 1582

Sana, H., Gosset, E., \& Evans, C. J. 2009, MNRAS, 400, 1479

Sánchez-Bermúdez, J., Alberdi, A., Schödel, R., et al. 2014, A\&A, 572, L1

Schaefer, G. H., Hummel, C. A., Gies, D. R., et al. 2016, AJ, 152, 213

Schoettler, C., de Bruijne, J., Vaher, E., \& Parker, R. J. 2020, MNRAS, 495, 3104

Shi, H. M., \& Hu, J. Y. 1999, A\&AS, 136, 313

Shultz, M., Le Bouquin, J. B., Rivinius, T., et al. 2019, MNRAS, 482, 3950

Simón-Díaz, S., Herrero, A., Esteban, C., \& Najarro, F. 2006, A\&A, 448, 351

Simón-Díaz, S., Caballero, J. A., \& Lorenzo, J. 2011, ApJ, 742, 55

Simón-Díaz, S., Caballero, J. A., Lorenzo, J., et al. 2015, ApJ, 799, 169

Skiff, B. A. 2014, VizieR Online Data Catalog: B/mk
Slettebak, A. 1963, ApJ, 138, 118

Smith, N. 2006a, MNRAS, 367, 763

Smith, N. 2006b, ApJ, 644, 1151

Smith, N., \& Frew, D. J. 2011, MNRAS, 415, 2009

Sota, A., Maíz Apellániz, J., Walborn, N. R., et al. 2011, ApJS, 193, 24

Sota, A., Maíz Apellániz, J., Morrell, N. I., et al. 2014, ApJS, 211, 10

Stickland, D. J. 1997, The Observatory, 117, 37

Stickland, D. J., Lloyd, C., \& Sweet, I. 1998, The Observatory, 118, 7

Sung, H., Bessell, M. S., \& Chun, M.-Y. 2004, AJ, 128, 1684

Sung, H., Bessell, M. S., Chun, M.-Y., et al. 2017, ApJS, 230, 3

Taylor, W. D., Evans, C. J., Simón-Díaz, S., et al. 2014, MNRAS, 442, 1483

Thompson, I. B., Udalski, A., Dotter, A., et al. 2020, MNRAS, 492, 4254

Tothill, N. F. H., Gagné, M., \& Stecklum, B. 2008, in The Lagoon Nebula and its Vicinity, ed. B. Reipurth, ASP, 5, 533

Trigueros Páez, E., Barbá, R. H., Negueruela, I., et al. 2021, A\&A, 655, A42

Trumpler, R. J. 1931, PASP, 43, 255

Turner, D. G. 1976, ApJ, 210, 65

van Leeuwen, F. 2007, Hipparcos, the New Reduction of the Raw Data (Springer

Science+Business Media B.V.), 350, 20

van Leeuwen, F., \& van Genderen, A. M. 1997, A\&A, 327, 1070

Vitrichenko, É. A. 2002, Astron. Lett., 28, 843

Walborn, N. R. 1982, AJ, 87, 1300

Walborn, N. R., Sota, A., Maíz Apellániz, J., et al. 2010, ApJ, 711, L143

Walborn, N. R., Sana, H., Simón-Díaz, S., et al. 2014, A\&A, 564, A40

Weidner, C., \& Kroupa, P. 2006, MNRAS, 365, 1333

Williams, A. M., Gies, D. R., Bagnuolo, W. G., Jr, et al. 2001, ApJ, 548, 425

Wright, N. J., Jeffries, R. D., Jackson, R. J., et al. 2019, MNRAS, 486, 2477

Zapatero Osorio, M. R., Béjar, V. J. S., Pavlenko, Y., et al. 2002, A\&A, 384, 937

Zari, E., Brown, A. G. A., \& de Zeeuw, P. T. 2019, A\&A, 628, A123

Zucker, C., Speagle, J. S., Schlafly, E. F., et al. 2020, A\&A, 633, A51 
J. Maíz Apellániz et al.: Villafranca OB groups. II.

\section{Appendix A: Additional tables and figures}

In this appendix we present:

1. The field sizes and filters used to determine the parameters for the 26 stellar groups in this paper (Table A.1).

2. The main results for those 26 stellar groups (Table A.2).
3. The spectrograms from GOSSS and LiLiMaRlin that have not appeared in previous papers. Figures A.1 to A.17 show the first type and Figures A.18 to A.23 the second type, in both cases sorted by the Villafranca group the belong to.

4. The corresponding spectral classifications in Tables A.3, A.3, and A.4.

Table A.1. Field sizes and filters applied to the O-type stellar groups and subgroups in this paper.

\begin{tabular}{|c|c|c|c|c|c|c|c|c|c|c|}
\hline ID & $N_{\mathrm{f}}$ & field & $\begin{array}{r}\alpha \\
(\operatorname{deg})\end{array}$ & $\begin{array}{r}\delta \\
(\mathrm{deg}) \\
\end{array}$ & $\begin{array}{r}r \\
\left({ }^{\prime \prime}\right) \\
\end{array}$ & $\begin{array}{c}\mu_{\alpha *} \\
\text { (mas/a) }\end{array}$ & $\begin{array}{c}\mu_{\delta} \\
\text { (mas/a) }\end{array}$ & $\begin{array}{c}r_{\mu} \\
\text { (mas/a) }\end{array}$ & $\Delta\left(G_{\mathrm{BP}}^{\prime}-G_{\mathrm{RP}}^{\prime}\right)$ & $G_{\max }^{\prime}$ \\
\hline O-001 & 28594 & $20^{\prime} \times 20^{\prime}$ & 168.79 & -61.26 & 206 & -5.61 & +1.97 & 0.44 & $>-0.34$ & - \\
\hline O-002 & 15183 & $20^{\prime} \times 20^{\prime}$ & 160.95 & -59.56 & 171 & -6.45 & +2.25 & 0.70 & $>-0.69$ & - \\
\hline $\mathrm{O}-003$ & 19709 & $20^{\prime} \times 20^{\prime}$ & 161.09 & -59.73 & 103 & -7.10 & +2.80 & 0.73 & $>-1.30$ & - \\
\hline O-004 & 62124 & $40^{\prime} \times 40^{\prime}$ & 155.99 & -57.76 & 400 & -5.10 & +2.82 & 0.70 & $>-0.65$ & - \\
\hline O-005 & 10999 & $30^{\prime} \times 30^{\prime}$ & 261.18 & -34.21 & 605 & -1.16 & -2.26 & 1.40 & $>-1.00$ & - \\
\hline O-006 & 49576 & $20^{\prime} \times 20^{\prime}$ & 164.68 & -61.18 & 200 & -5.50 & +2.30 & 0.30 & $>-0.90$ & - \\
\hline O-007 & 5361 & $20^{\prime} \times 20^{\prime}$ & 308.30 & +41.22 & 186 & -2.65 & -4.49 & 0.72 & $>-0.60$ & - \\
\hline O-008 & 6299 & $20^{\prime} \times 20^{\prime}$ & 308.32 & +41.31 & 126 & -2.67 & -4.15 & 0.50 & $>-0.80$ & - \\
\hline O-009 & 5987 & $20^{\prime} \times 20^{\prime}$ & 275.12 & -16.18 & 236 & +0.10 & -1.52 & 1.10 & $>-0.70$ & - \\
\hline O-010 & 33947 & $20^{\prime} \times 20^{\prime}$ & 250.30 & -48.76 & 285 & +1.57 & -3.92 & 1.04 & $>-0.70$ & - \\
\hline O-011 & 6806 & $20^{\prime} \times 20^{\prime}$ & 308.83 & +46.84 & 341 & -2.78 & -4.34 & 0.50 & $>-0.28$ & - \\
\hline O-012 & 26170 & $30^{\prime} \times 30^{\prime}$ & 118.18 & -26.33 & 650 & -2.50 & +2.60 & 0.40 & $>-0.15$ & - \\
\hline $\mathrm{O}-012 \mathrm{~N}$ & 26170 & $30^{\prime} \times 30^{\prime}$ & 118.19 & -26.28 & 200 & -2.50 & +2.60 & 0.40 & $>-0.15$ & - \\
\hline O-012 S & 26170 & $30^{\prime} \times 30^{\prime}$ & 118.18 & -26.42 & 320 & -2.50 & +2.60 & 0.40 & $>-0.15$ & - \\
\hline O-013 & 3266 & $20^{\prime} \times 20^{\prime}$ & 348.43 & +61.50 & 109 & -3.70 & -2.14 & 0.73 & $>-0.20$ & - \\
\hline O-014 SE & 291324 & $120^{\prime} \times 120^{\prime}$ & 314.56 & +43.84 & 420 & -0.50 & -3.50 & 1.30 & $>+0.00$ & - \\
\hline O-014 NW & 291324 & $120^{\prime} \times 120^{\prime}$ & 313.10 & +44.40 & 1600 & -1.75 & -3.05 & 0.70 & $>+0.60$ & - \\
\hline O-015 & 19903 & $30^{\prime} \times 30^{\prime}$ & 304.60 & +40.78 & 800 & -2.60 & -6.40 & 0.75 & $>-0.30$ & - \\
\hline O-016 & 26017 & $60^{\prime} \times 60^{\prime}$ & 100.25 & +09.75 & 1500 & -1.80 & -3.70 & 1.50 & $>-0.20$ & - \\
\hline $\mathrm{O}-016 \mathrm{~N}$ & 26017 & $60^{\prime} \times 60^{\prime}$ & 100.20 & +09.88 & 540 & -1.80 & -3.70 & 1.50 & $>-0.20$ & - \\
\hline $\mathrm{O}-016 \mathrm{~S}$ & 26017 & $60^{\prime} \times 60^{\prime}$ & 100.28 & +09.53 & 540 & -1.80 & -3.70 & 1.50 & $>-0.20$ & - \\
\hline O-017 & 59219 & $60^{\prime} \times 60^{\prime}$ & 38.17 & +61.46 & 1400 & -0.90 & -0.50 & 0.75 & $>-0.30$ & - \\
\hline O-018 & 243382 & $60^{\prime} \times 60^{\prime}$ & 271.10 & -24.40 & 1000 & +1.50 & -2.00 & 1.40 & -0.30 to +2.50 & - \\
\hline O-019 & 11353 & $30^{\prime} \times 30^{\prime}$ & 274.68 & -13.79 & 800 & +0.20 & -1.70 & 1.00 & $>-0.43$ & - \\
\hline O-020 & 33691 & $60^{\prime} \times 60^{\prime}$ & 97.98 & +4.94 & 1200 & -1.80 & +0.30 & 1.00 & $>-0.70$ & - \\
\hline $\mathrm{O}-021$ & 10521 & $20^{\prime} \times 20^{\prime}$ & 109.68 & -24.95 & 400 & -2.80 & +3.10 & 1.00 & $>-0.37$ & - \\
\hline O-022 & 39632 & $30^{\prime} \times 30^{\prime}$ & 253.54 & -41.83 & 800 & -0.40 & -2.40 & 1.00 & -0.70 to +2.00 & 16.5 \\
\hline $\mathrm{O}-023$ & 6637 & $60^{\prime} \times 60^{\prime}$ & 83.82 & -5.39 & 700 & +1.50 & +0.50 & 1.30 & $>-0.62$ & - \\
\hline $\mathrm{O}-024$ & 82518 & $60^{\prime} \times 60^{\prime}$ & 122.38 & -47.33 & 1500 & -6.60 & +9.80 & 1.35 & $>-0.15$ & - \\
\hline $\mathrm{O}-025$ & 18153 & $20^{\prime} \times 20^{\prime}$ & 161.30 & -59.70 & 250 & -6.80 & +2.48 & 0.65 & $>-0.35$ & - \\
\hline O-026 & 14576 & $60^{\prime} \times 60^{\prime}$ & 84.69 & -2.60 & 600 & +1.10 & -0.60 & 1.00 & $>-0.07$ & - \\
\hline
\end{tabular}

RUWE is filtered as $<1.4, C^{*}$ as $<0.4$, and $\sigma_{\text {ext }}$ as $<0.1$ mas in all cases. 
Table A.2. Membership and distance results.

\begin{tabular}{|c|c|c|c|c|c|c|c|c|c|}
\hline ID & $N_{*, 0}$ & $N_{*}$ & $t_{\varpi}$ & $t_{\mu_{\alpha *}}$ & $t_{\mu_{\delta}}$ & $\begin{array}{c}\mu_{\alpha *, \mathrm{~g}} \\
(\mathrm{mas} / \mathrm{a})\end{array}$ & $\begin{array}{c}\mu_{\delta, \mathrm{g}} \\
\text { (mas/a) }\end{array}$ & $\begin{array}{c}\varpi_{\mathrm{g}} \\
\text { (mas) }\end{array}$ & $\begin{array}{c}d \\
(\mathrm{pc})\end{array}$ \\
\hline O-001 & 170 & 166 & 0.91 & 2.93 & 2.21 & $-5.581 \pm 0.023$ & $+2.004 \pm 0.023$ & $0.141 \pm 0.011$ & $7130_{-500}^{+590}$ \\
\hline O-002 & 186 & 184 & 0.83 & 4.51 & 4.49 & $-6.534 \pm 0.023$ & $+2.076 \pm 0.023$ & $0.424 \pm 0.011$ & $2363_{-58}^{+61}$ \\
\hline $\mathrm{O}-003$ & 31 & 29 & 0.78 & 3.11 & 2.73 & $-7.128 \pm 0.024$ & $+2.670 \pm 0.024$ & $0.435 \pm 0.012$ & $2305_{-61}^{+64}$ \\
\hline O-004 & 114 & 110 & 0.95 & 3.27 & 3.68 & $-5.297 \pm 0.023$ & $+2.890 \pm 0.023$ & $0.226 \pm 0.011$ & $4440_{-210}^{+230}$ \\
\hline O-005 & 118 & 111 & 1.15 & 6.27 & 9.25 & $-0.898 \pm 0.023$ & $-2.221 \pm 0.023$ & $0.610 \pm 0.011$ & $\begin{array}{l}1642_{-29}^{+30} \\
\text { - }\end{array}$ \\
\hline O-006 & 97 & 96 & 0.97 & 1.93 & 2.03 & $-5.511 \pm 0.023$ & $+2.285 \pm 0.023$ & $0.137 \pm 0.011$ & $7260_{-520}^{+610}$ \\
\hline O-007 & 51 & 51 & 1.10 & 4.88 & 3.86 & $-2.725 \pm 0.024$ & $-4.448 \pm 0.024$ & $0.618 \pm 0.011$ & $1620_{-29}^{+30}$ \\
\hline O-008 & 40 & 40 & 0.93 & 3.18 & 3.54 & $-2.659 \pm 0.024$ & $-4.400 \pm 0.024$ & $0.623 \pm 0.011$ & $1608_{-29}^{+30}$ \\
\hline O-009 & 27 & 25 & 0.98 & 4.98 & 7.70 & $+0.303 \pm 0.026$ & $-1.523 \pm 0.024$ & $0.591 \pm 0.014$ & $1696_{-39}^{+41}$ \\
\hline O-010 & 71 & 68 & 1.00 & 4.66 & 5.29 & $+1.225 \pm 0.024$ & $-3.967 \pm 0.023$ & $0.872 \pm 0.012$ & $1148_{-15}^{+16}$ \\
\hline O-011 & 68 & 66 & 0.97 & 2.90 & 2.46 & $-2.855 \pm 0.023$ & $-4.391 \pm 0.023$ & $0.365 \pm 0.011$ & $2741_{-81}^{+86}$ \\
\hline O-012 & 258 & 228 & 0.99 & 3.01 & 3.00 & $-2.499 \pm 0.022$ & $+2.605 \pm 0.022$ & $0.237 \pm 0.011$ & $4241_{-180}^{+200}$ \\
\hline $\mathrm{O}-012 \mathrm{~N}$ & 63 & 61 & 0.87 & 1.63 & 1.71 & $-2.528 \pm 0.024$ & $+2.502 \pm 0.024$ & $0.231 \pm 0.011$ & $4350_{-210}^{+230}$ \\
\hline O-012 S & 100 & 90 & 0.94 & 2.98 & 2.80 & $-2.506 \pm 0.023$ & $+2.667 \pm 0.023$ & $0.236 \pm 0.011$ & $4250_{-190}^{+210}$ \\
\hline O-013 & 11 & 11 & 0.86 & 3.47 & 5.67 & $-3.668 \pm 0.026$ & $-2.301 \pm 0.026$ & $0.370 \pm 0.015$ & $2710_{-100}^{+110}$ \\
\hline O-014 SE & 13 & 13 & 1.09 & 5.86 & 5.16 & $-0.653 \pm 0.026$ & $-3.103 \pm 0.026$ & $1.344 \pm 0.016$ & $744_{-9}^{+9}$ \\
\hline O-014 NW & 248 & 144 & 1.01 & 7.45 & 6.05 & $-1.370 \pm 0.020$ & $-3.054 \pm 0.020$ & $1.254 \pm 0.010$ & $798_{-6}^{+6}$ \\
\hline O-015 & 140 & 102 & 1.17 & 4.60 & 3.70 & $-2.570 \pm 0.022$ & $-6.370 \pm 0.022$ & $1.000 \pm 0.011$ & $1001_{-11}^{+11}$ \\
\hline O-016 & 399 & 340 & 0.88 & 8.04 & 5.27 & $-1.852 \pm 0.020$ & $-3.688 \pm 0.020$ & $1.423 \pm 0.010$ & $703_{-5}^{+5}$ \\
\hline O-016 N & 123 & 119 & 0.87 & 5.83 & 4.52 & $-1.673 \pm 0.023$ & $-3.695 \pm 0.023$ & $1.426 \pm 0.011$ & $701_{-5}^{+6}$ \\
\hline O-016 S & 109 & 107 & 0.96 & 8.12 & 4.94 & $-2.026 \pm 0.023$ & $-3.759 \pm 0.023$ & $1.416 \pm 0.011$ & $706_{-6}^{+6}$ \\
\hline O-017 & 480 & 343 & 1.22 & 5.46 & 5.52 & $-0.843 \pm 0.020$ & $-0.537 \pm 0.020$ & $0.483 \pm 0.010$ & $2075_{-42}^{+44}$ \\
\hline O-018 & 388 & 301 & 1.10 & 8.24 & 8.53 & $+1.385 \pm 0.022$ & $-2.024 \pm 0.022$ & $0.811 \pm 0.010$ & $1234_{-16}^{+16}$ \\
\hline O-019 & 202 & 188 & 0.96 & 5.26 & 7.16 & $+0.200 \pm 0.022$ & $-1.627 \pm 0.022$ & $0.590 \pm 0.011$ & $1697_{-30}^{+31}$ \\
\hline $\mathrm{O}-020$ & 268 & 223 & 1.07 & 4.83 & 6.79 & $-1.729 \pm 0.021$ & $+0.276 \pm 0.021$ & $0.704 \pm 0.010$ & $1421_{-20}^{+21}$ \\
\hline O-021 & 129 & 116 & 1.09 & 5.15 & 3.51 & $-2.776 \pm 0.023$ & $+2.990 \pm 0.023$ & $0.815 \pm 0.011$ & $1227_{-16}^{+17}$ \\
\hline $\mathrm{O}-022$ & 527 & 495 & 1.01 & 5.08 & 5.72 & $-0.544 \pm 0.022$ & $-2.210 \pm 0.022$ & $0.645 \pm 0.010$ & $1551_{-24}^{+25}$ \\
\hline $\mathrm{O}-023$ & 84 & 82 & 0.92 & 11.29 & 12.49 & $+1.577 \pm 0.022$ & $+0.464 \pm 0.022$ & $2.562 \pm 0.011$ & $390_{-2}^{+2}$ \\
\hline $\mathrm{O}-024$ & 167 & 138 & 1.22 & 7.03 & 6.44 & $-6.560 \pm 0.020$ & $+9.802 \pm 0.020$ & $2.974 \pm 0.010$ & $336_{-1}^{+1}$ \\
\hline $\mathrm{O}-025$ & 177 & 175 & 0.90 & 4.11 & 3.77 & $-6.877 \pm 0.023$ & $+2.596 \pm 0.023$ & $0.434 \pm 0.011$ & $2311_{-56}^{+58}$ \\
\hline O-026 & 60 & 55 & 0.99 & 7.16 & 6.81 & $+1.350 \pm 0.023$ & $-0.568 \pm 0.023$ & $2.520 \pm 0.012$ & $397_{-2}^{+2}$ \\
\hline
\end{tabular}


J. Maíz Apellániz et al.: Villafranca OB groups. II.

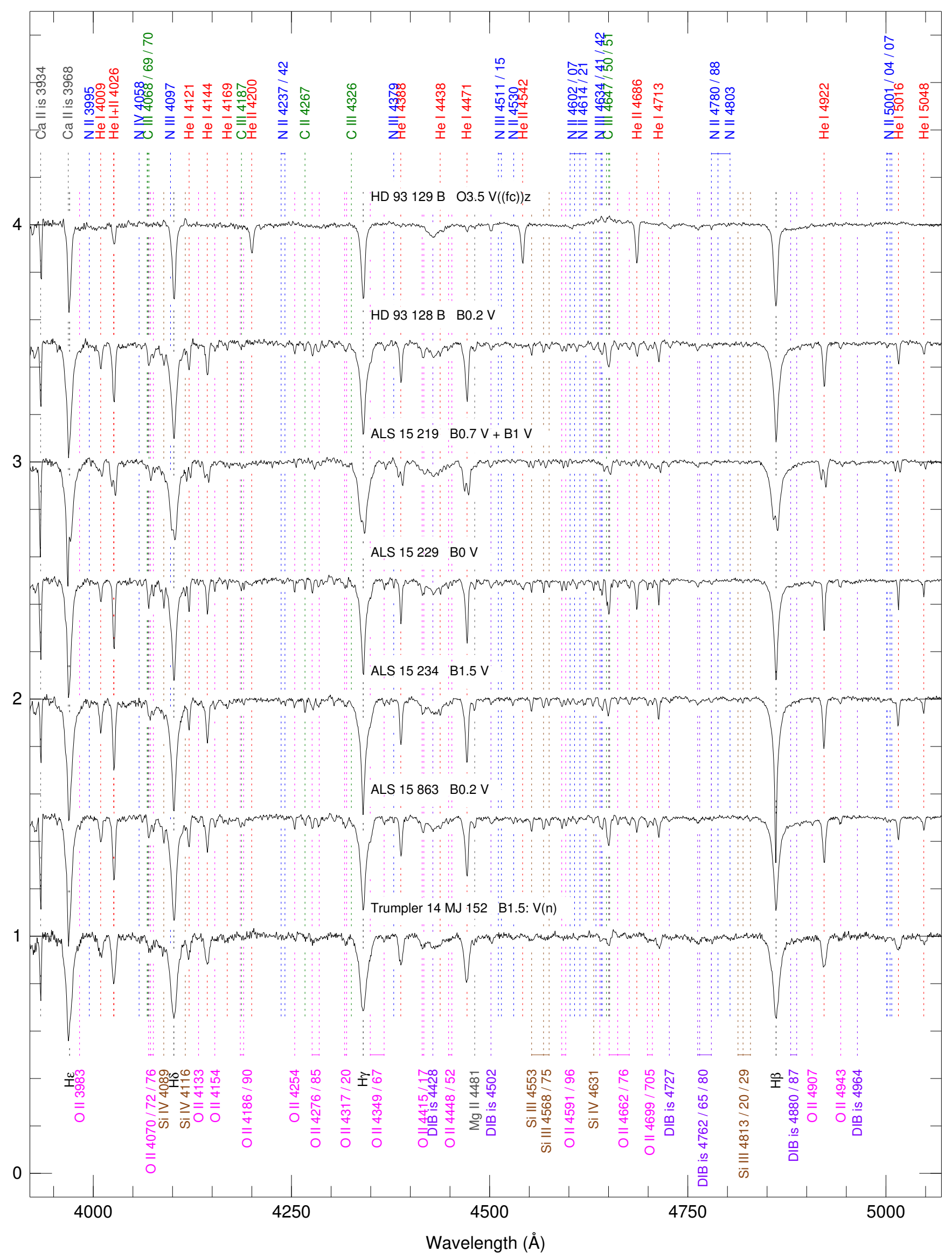

Fig. A.1. New GOSSS spectra for stars in Villafranca O-002. 


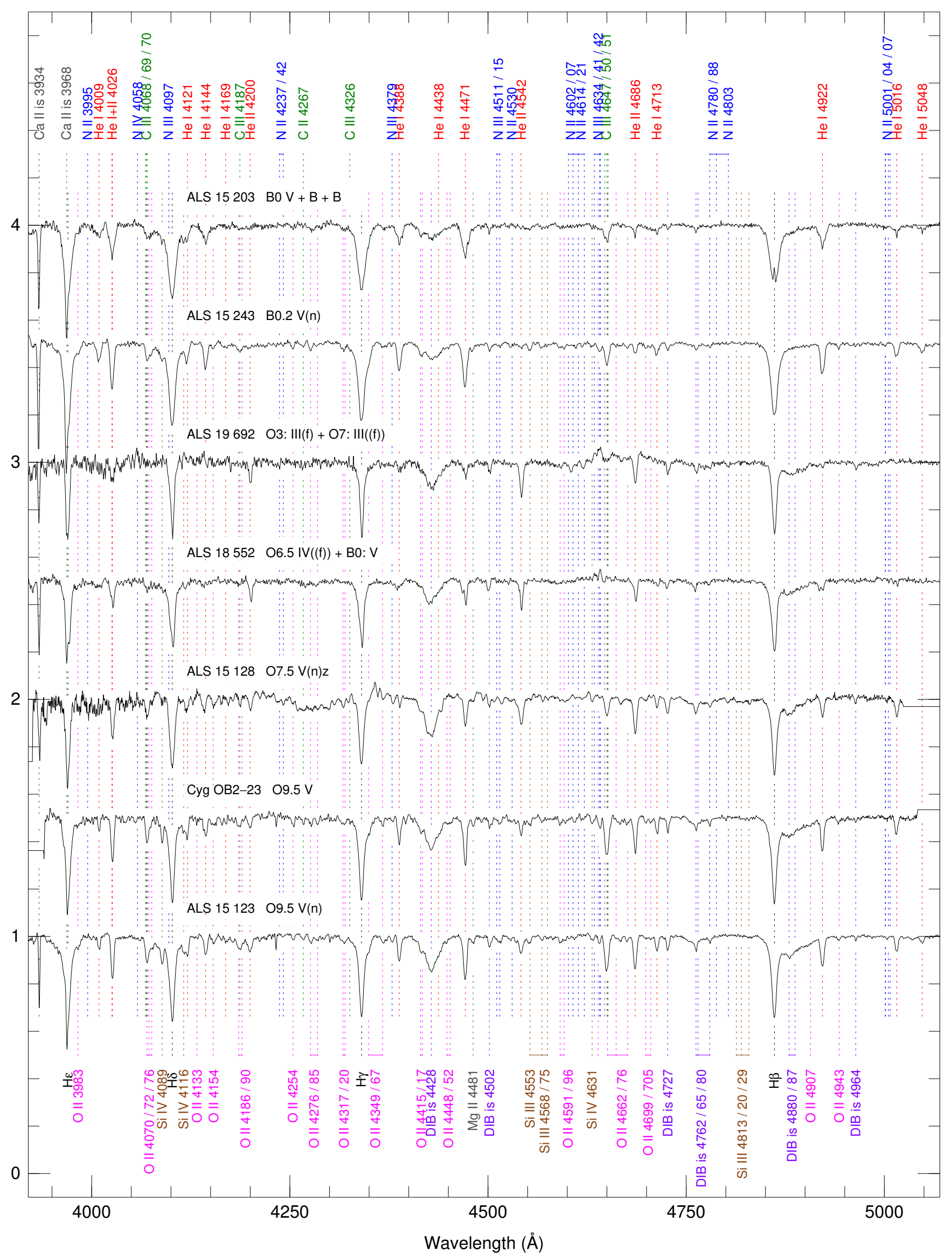

Fig. A.2. New GOSSS spectra for stars in Villafranca O-002, Villafranca O-003, Villafranca O-005, Villafranca O-006, Villafranca O-007, and Villafranca O-008. 
J. Maíz Apellániz et al.: Villafranca OB groups. II.

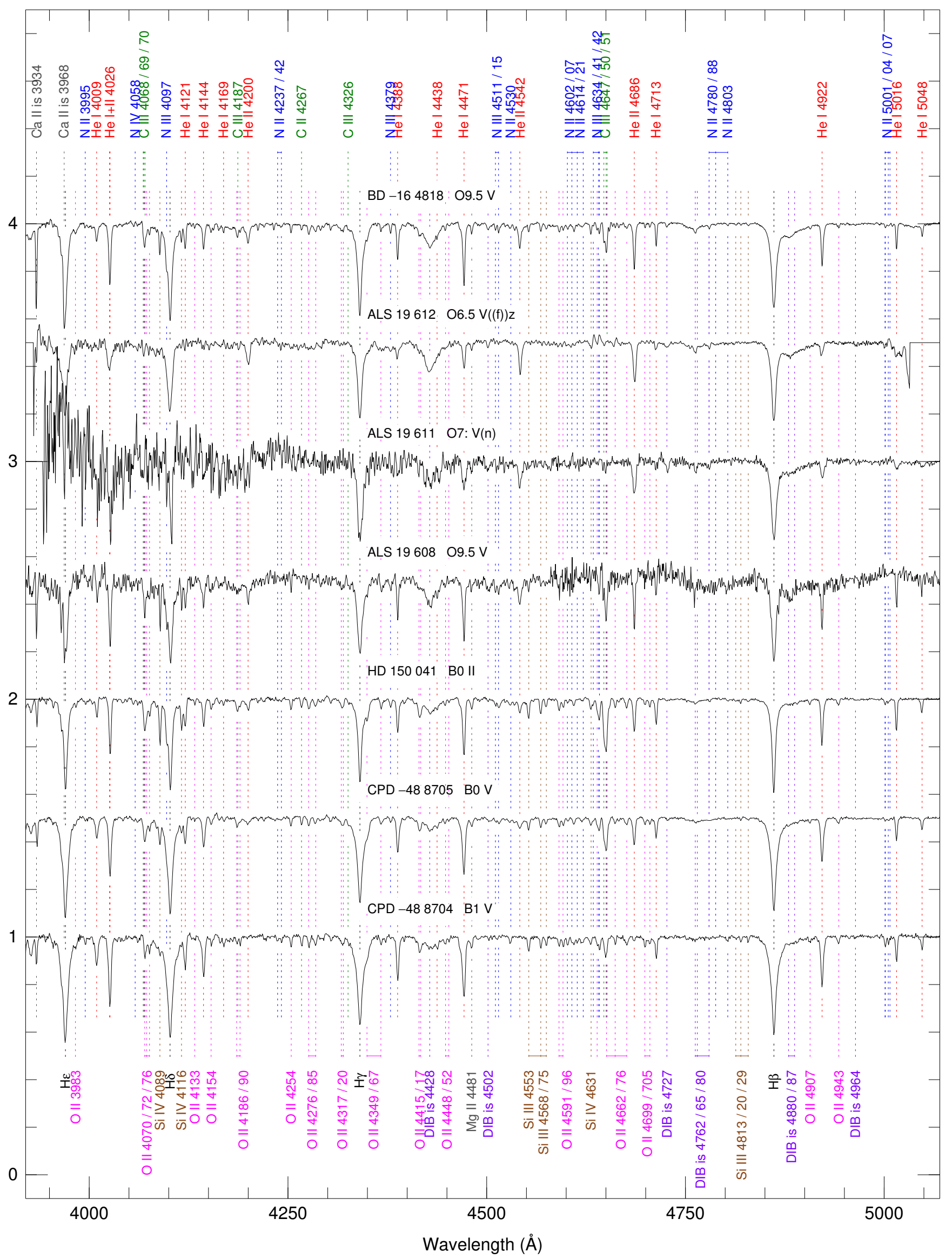

Fig. A.3. New GOSSS spectra for stars in Villafranca O-009 and Villafranca O-010. 


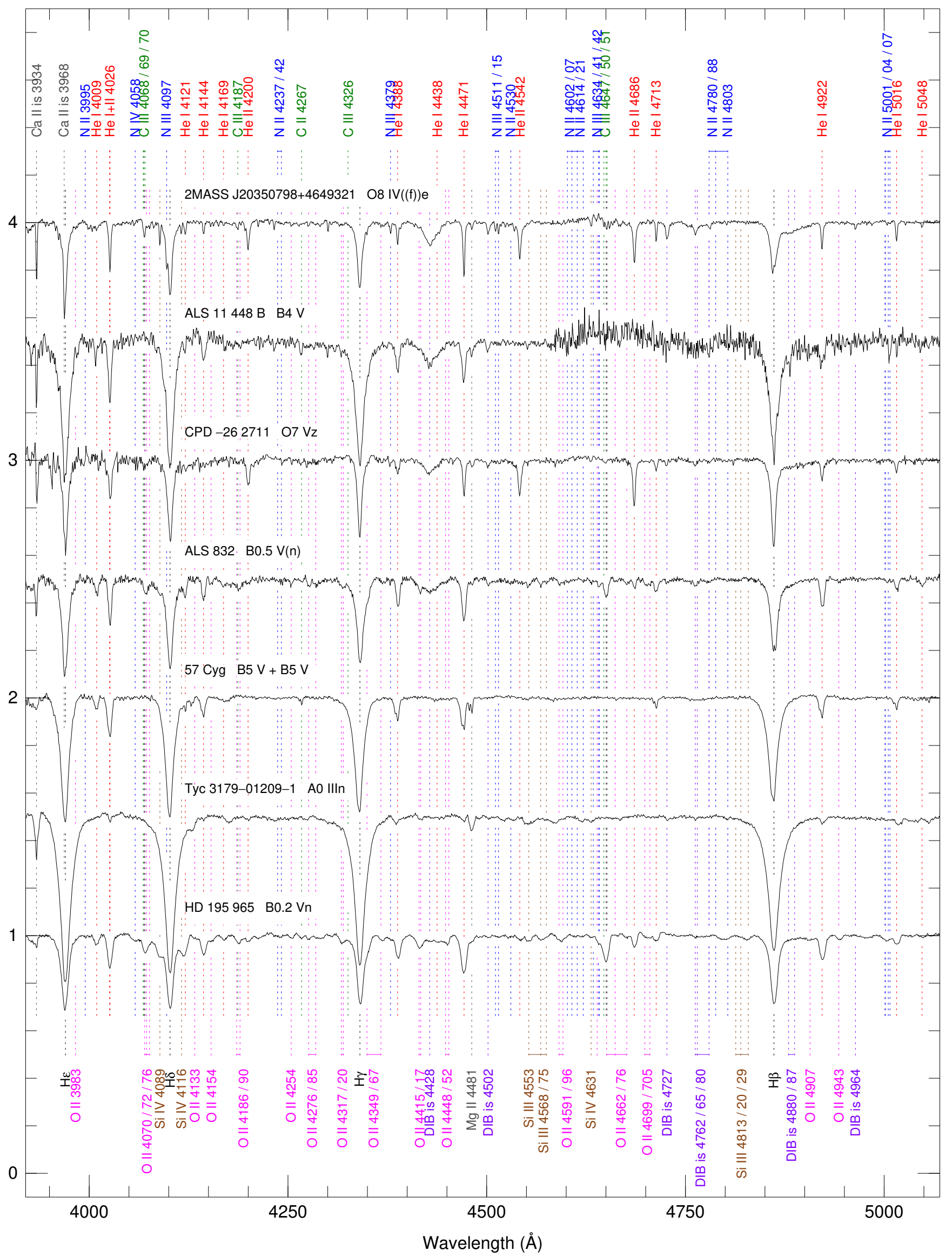

Fig. A.4. New GOSSS spectra for stars in Villafranca O-011, Villafranca O-012, and Villafranca O-014. 
J. Maíz Apellániz et al.: Villafranca OB groups. II.

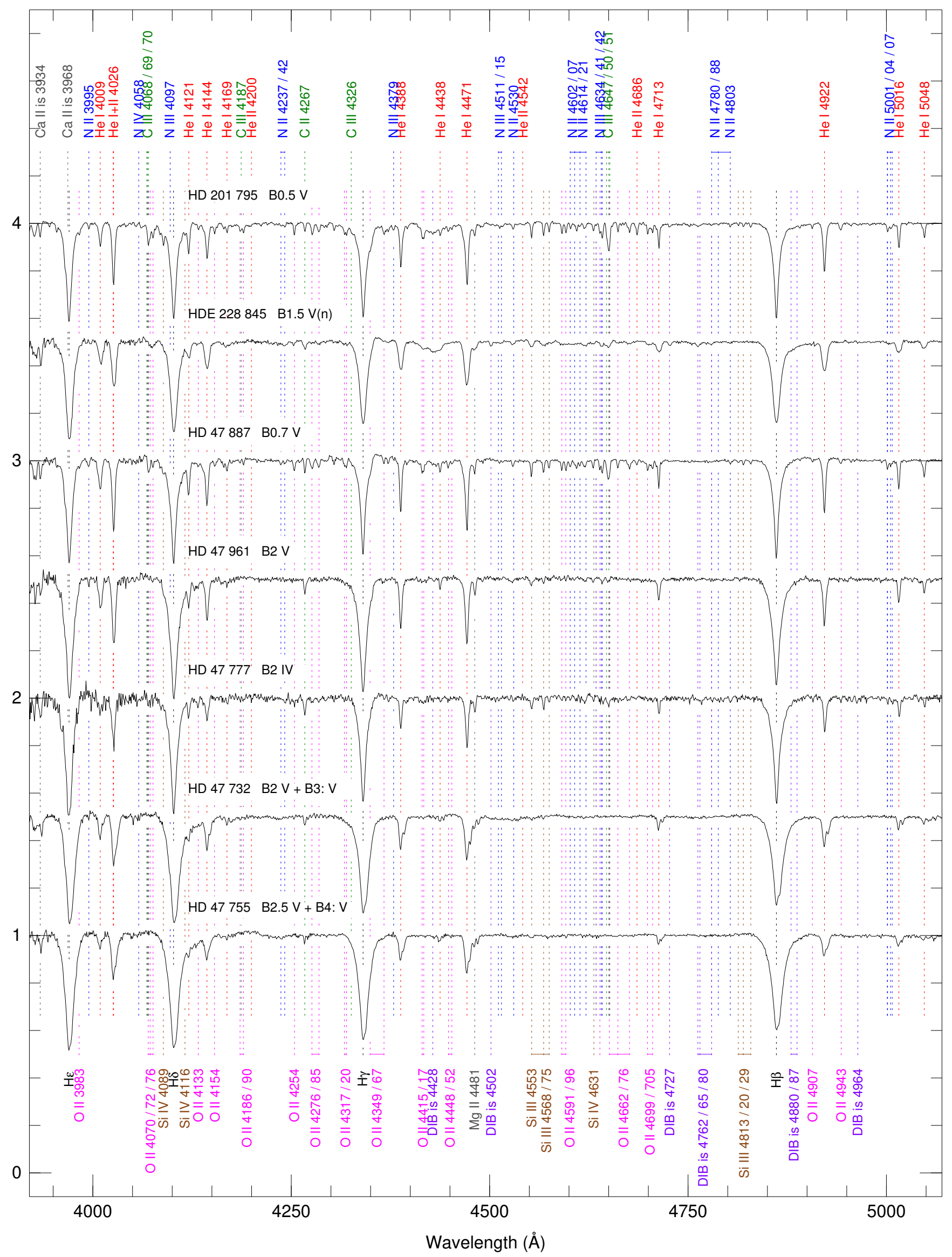

Fig. A.5. New GOSSS spectra for stars in Villafranca O-014, Villafranca O-015, and Villafranca O-016. 


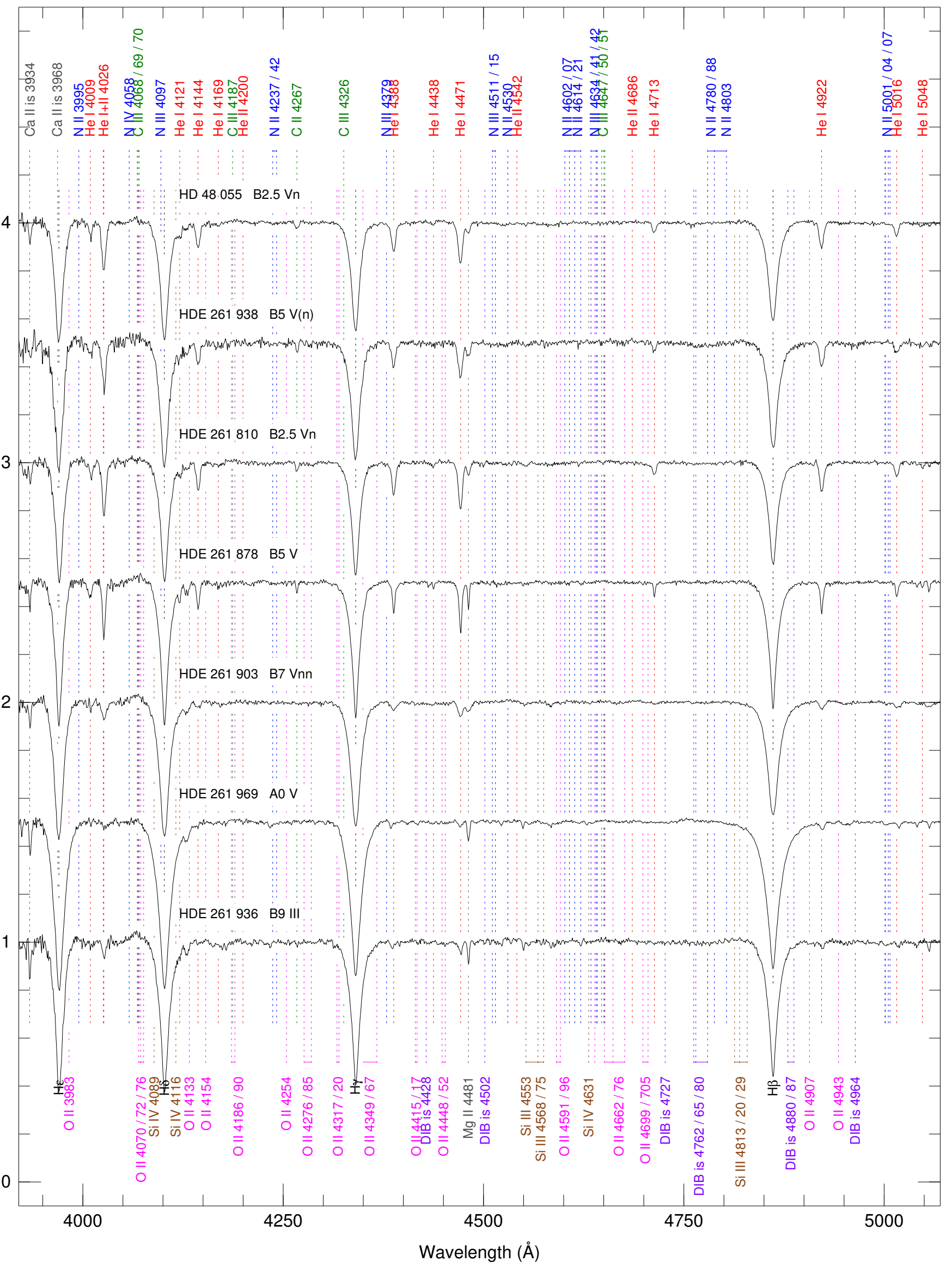

Fig. A.6. New GOSSS spectra for stars in Villafranca O-016. 
J. Maíz Apellániz et al.: Villafranca OB groups. II.

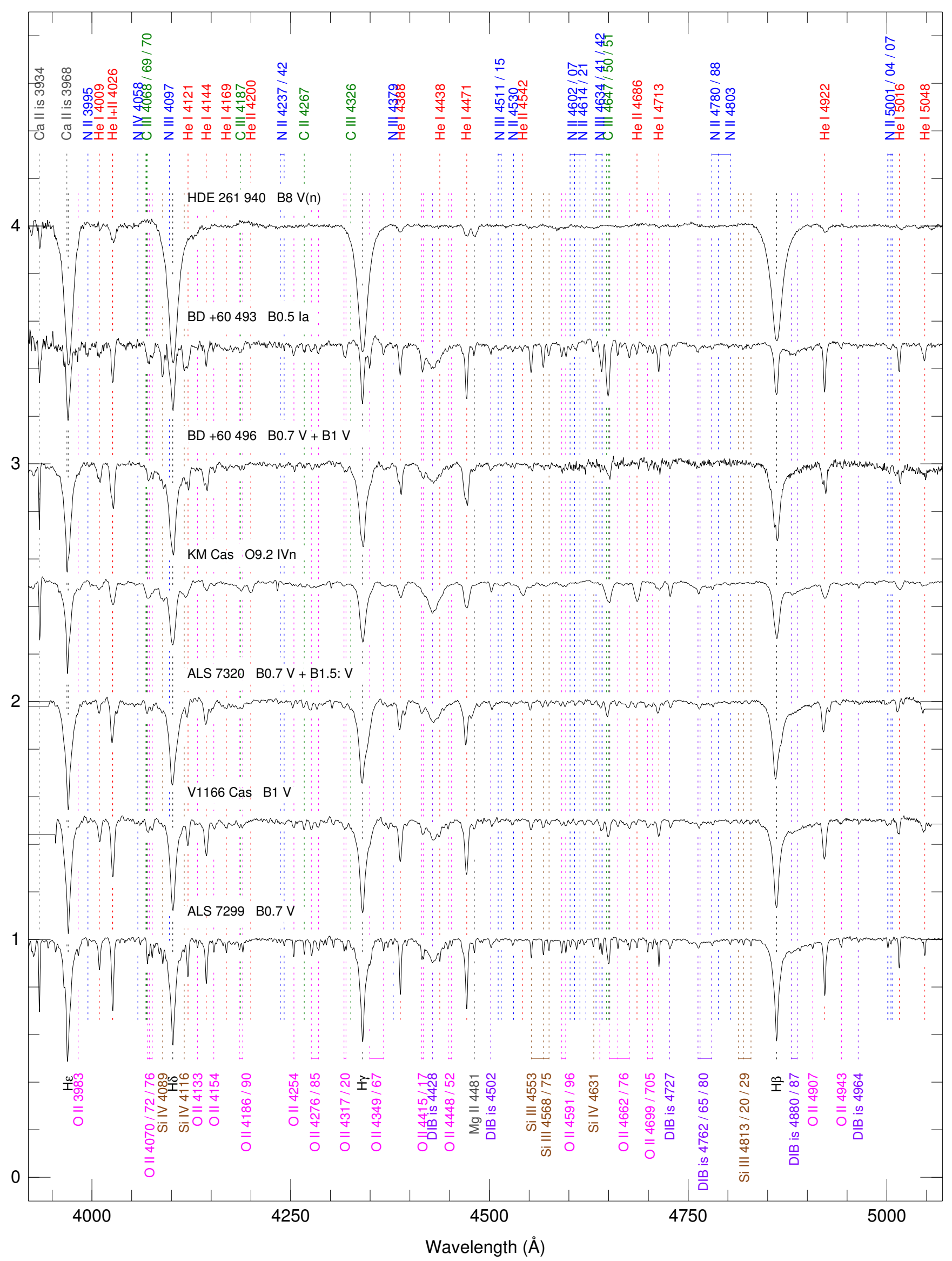

Fig. A.7. New GOSSS spectra for stars in Villafranca O-016 and Villafranca O-017. 


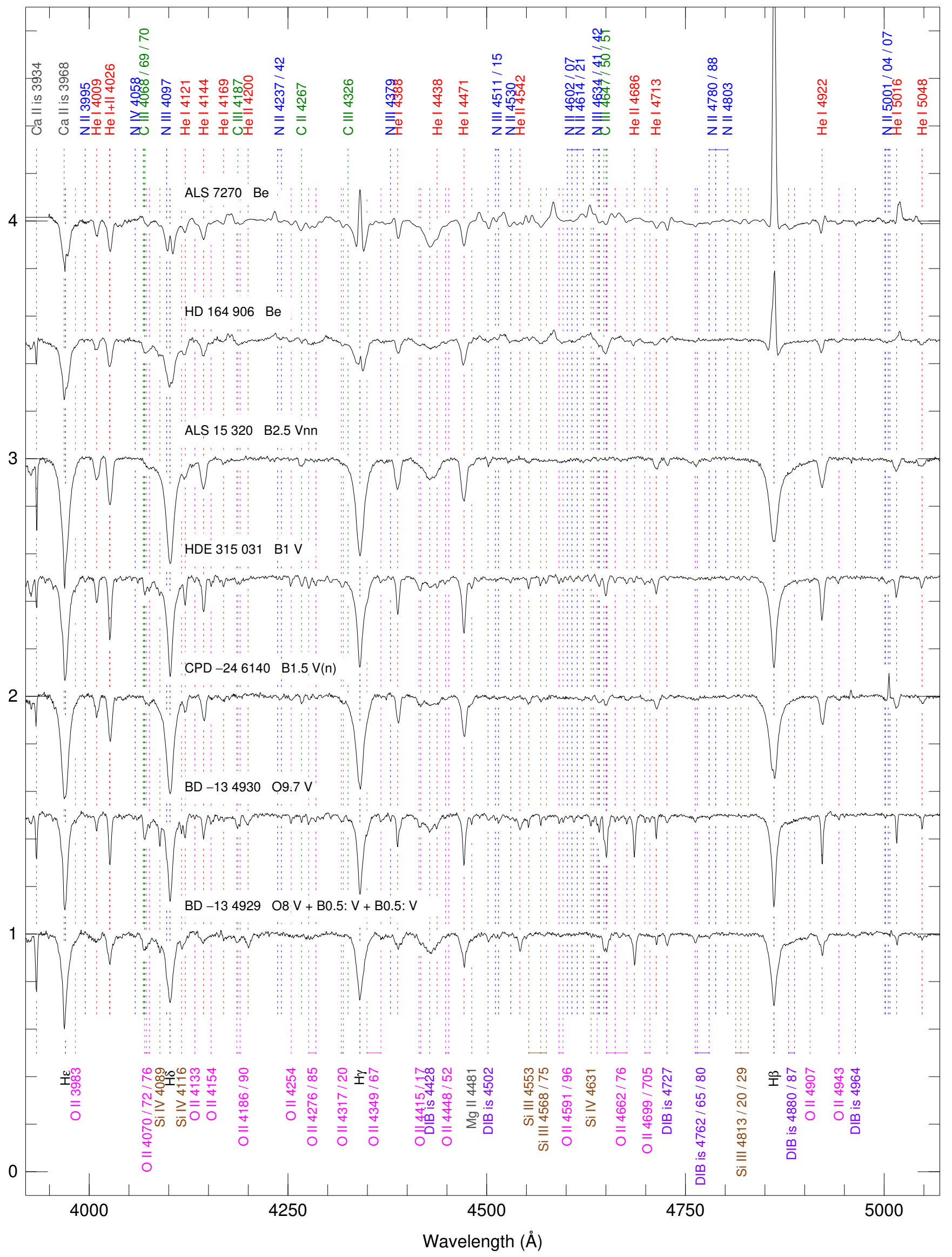

Fig. A.8. New GOSSS spectra for stars in Villafranca O-017, Villafranca O-018, and Villafranca O-019. 
J. Maíz Apellániz et al.: Villafranca OB groups. II.

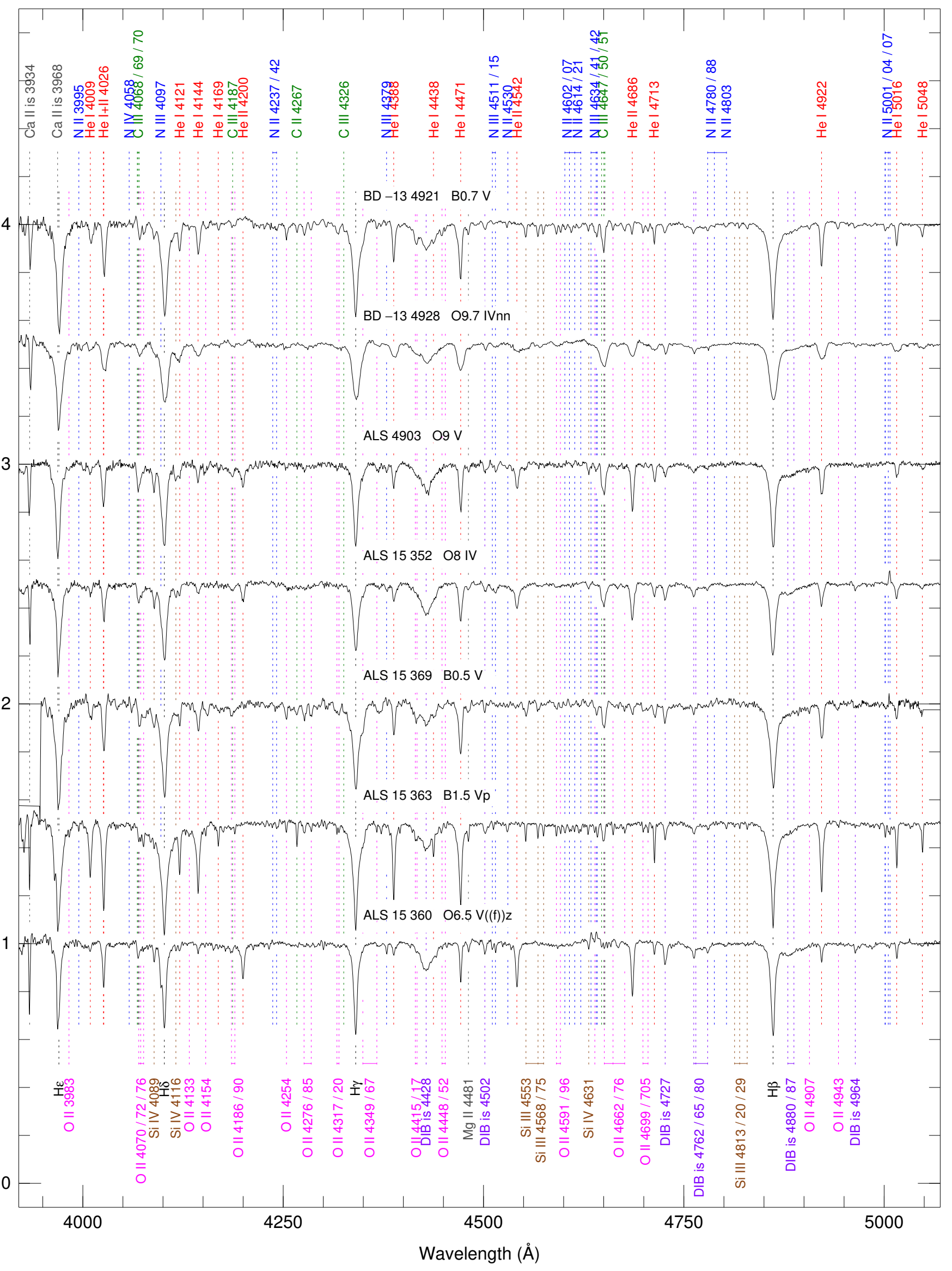

Fig. A.9. New GOSSS spectra for stars in Villafranca O-019. 
A\&A 657, A131 (2022)

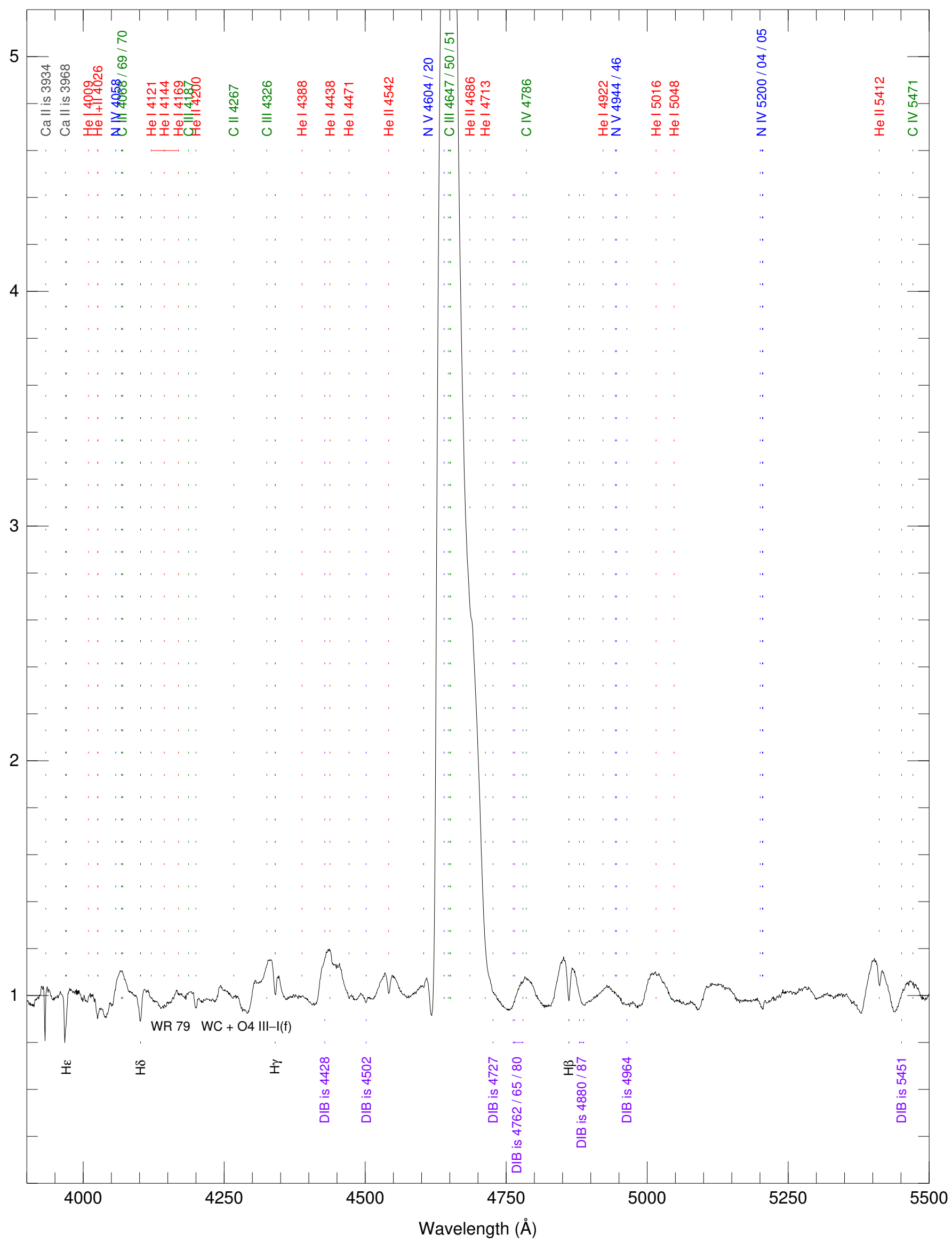

Fig. A.10. New GOSSS spectra for a star in Villafranca O-022. Note the expanded wavelength range. 
J. Maíz Apellániz et al.: Villafranca OB groups. II.

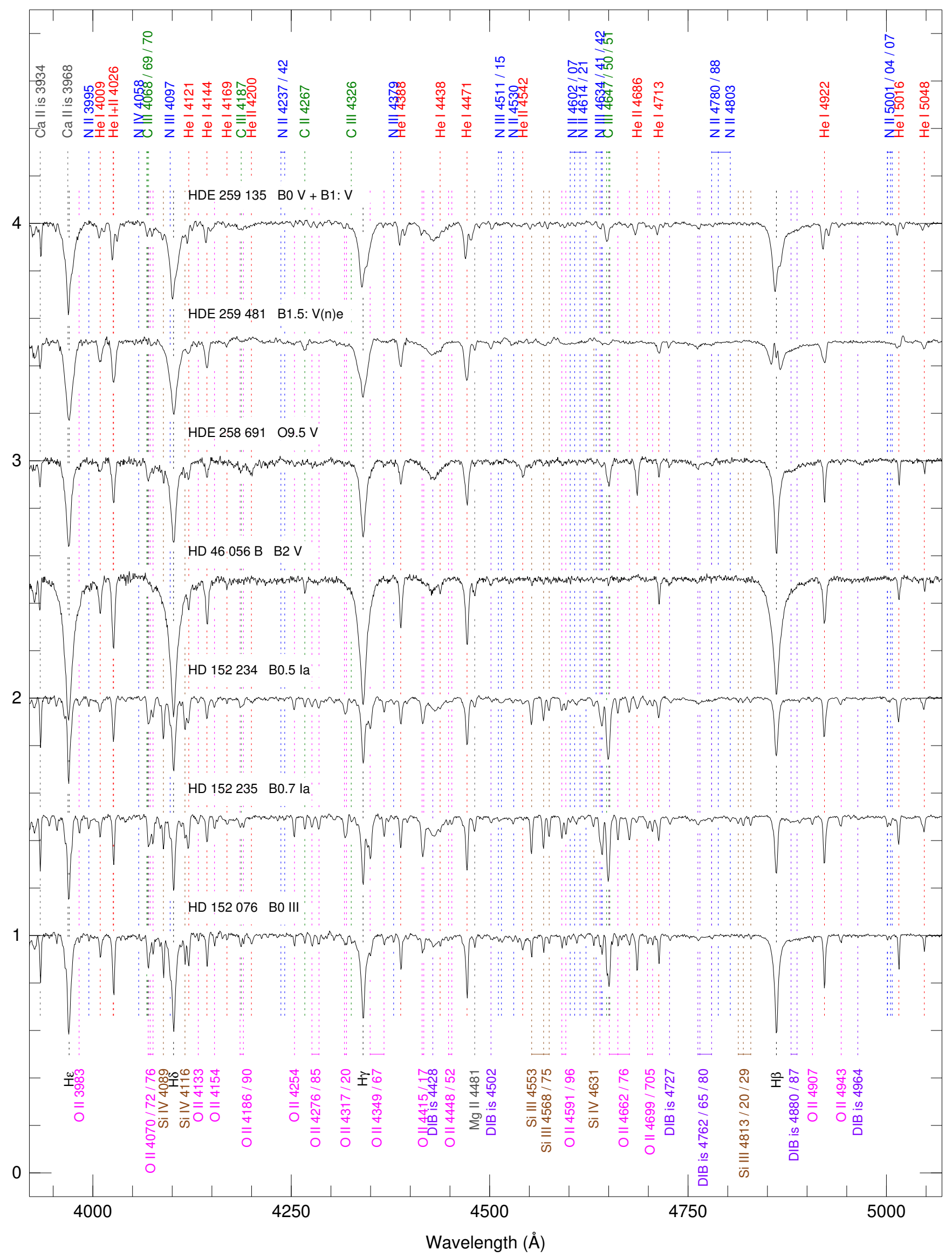

Fig. A.11. New GOSSS spectra for stars in Villafranca O-020 and Villafranca O-022. 


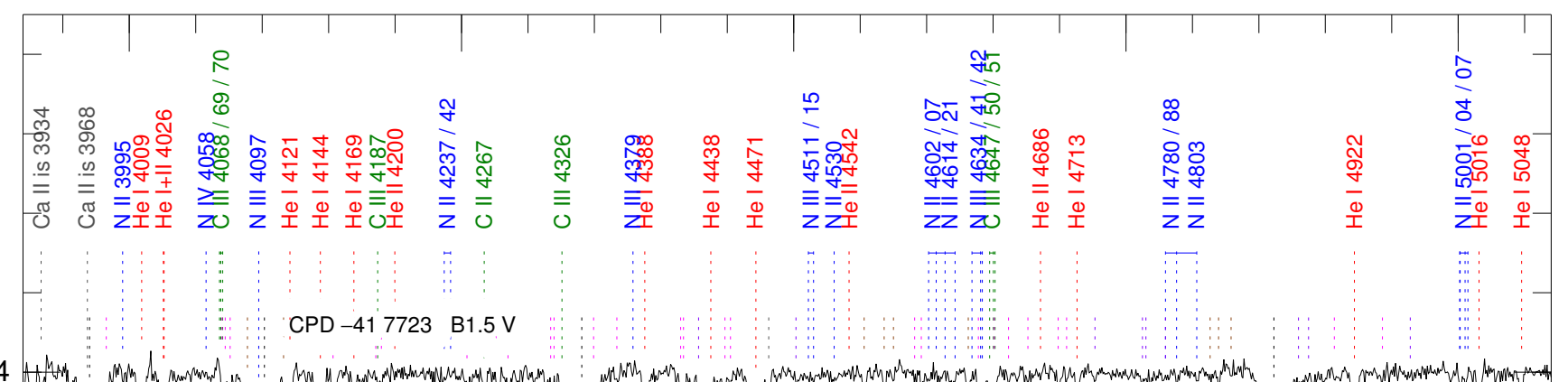

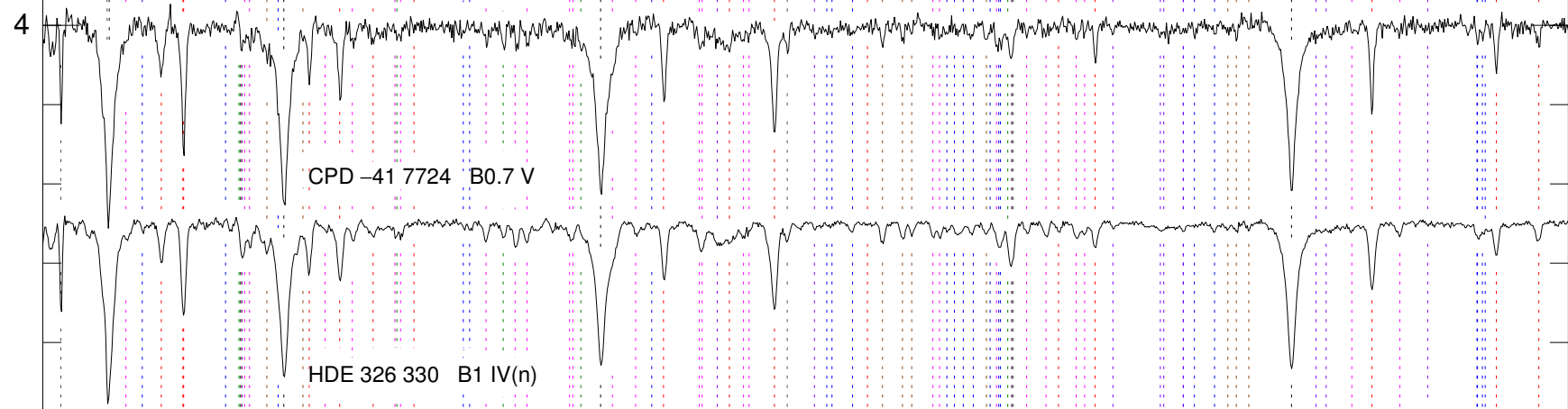
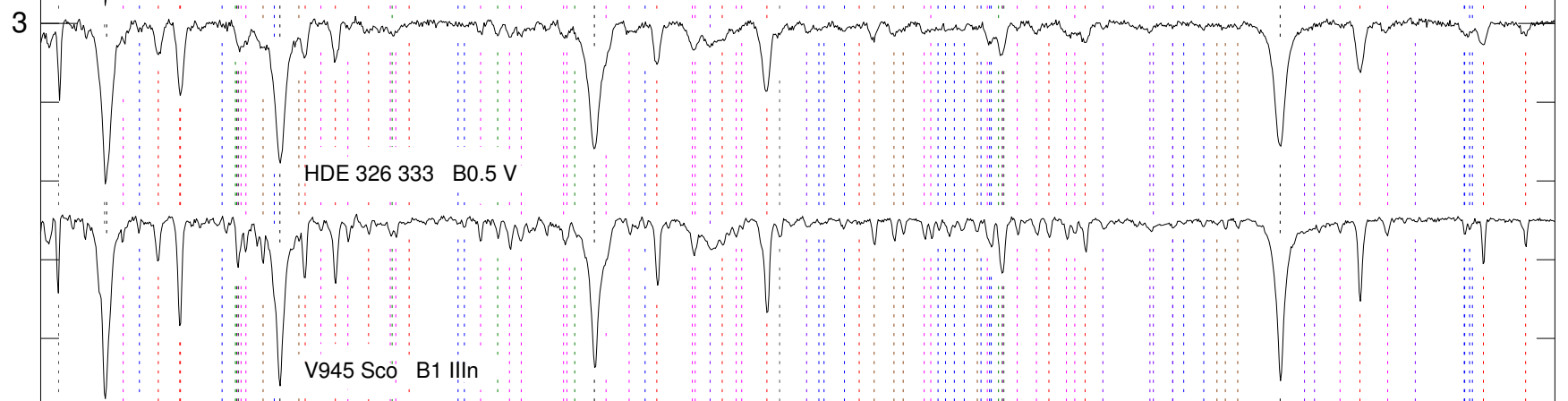

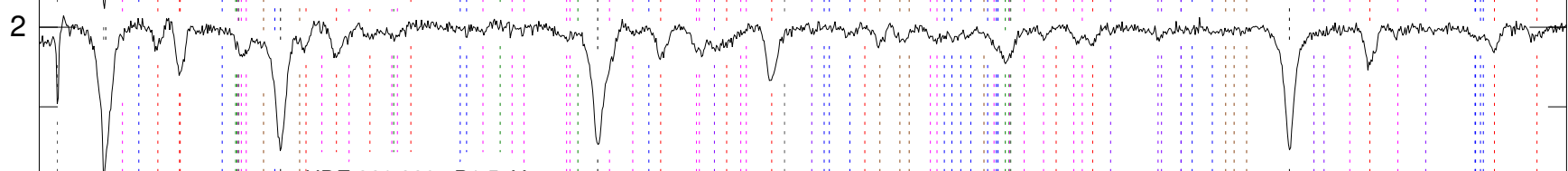

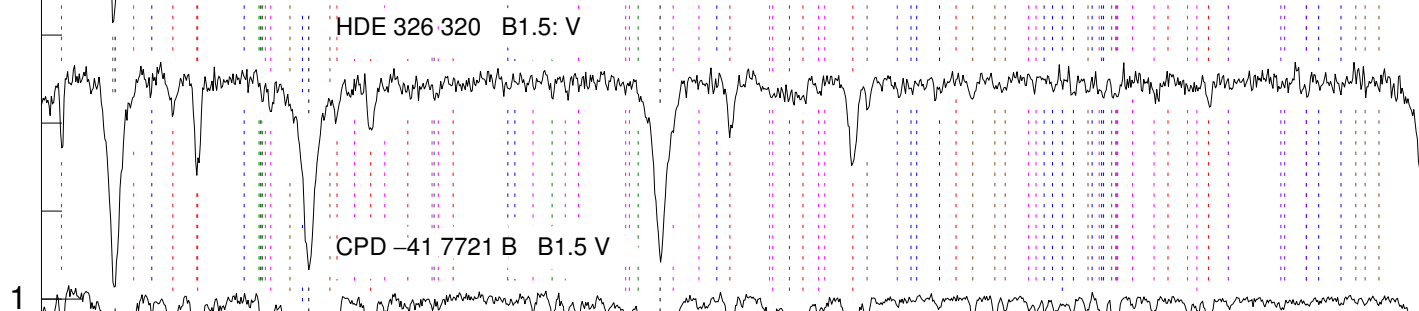

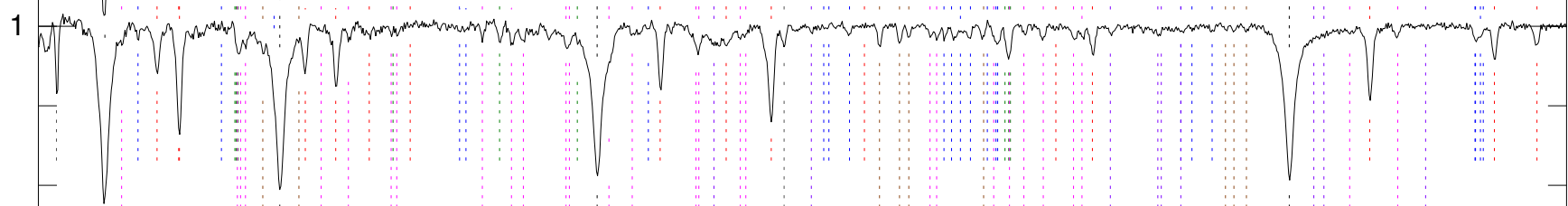

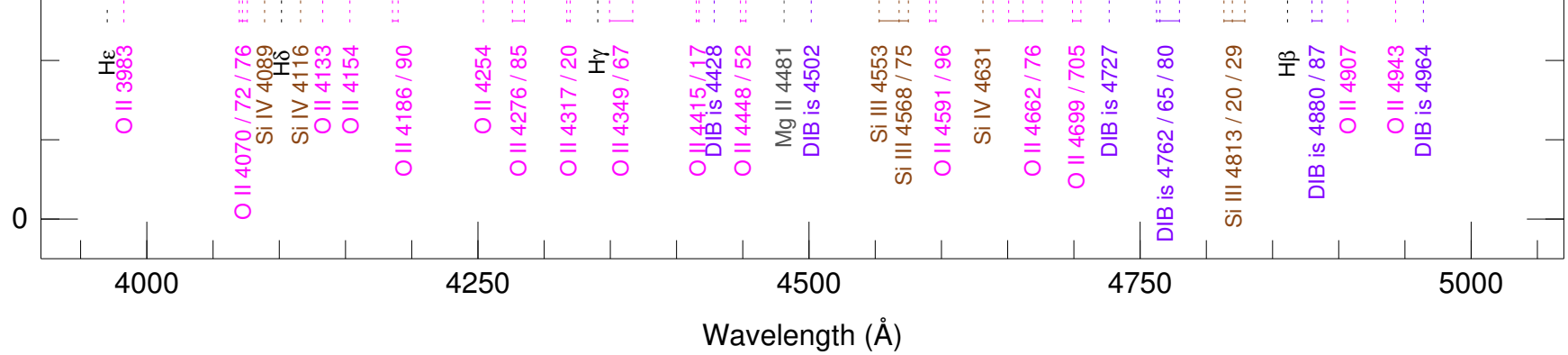

Fig. A.12. New GOSSS spectra for stars in Villafranca O-022. 
J. Maíz Apellániz et al.: Villafranca OB groups. II.

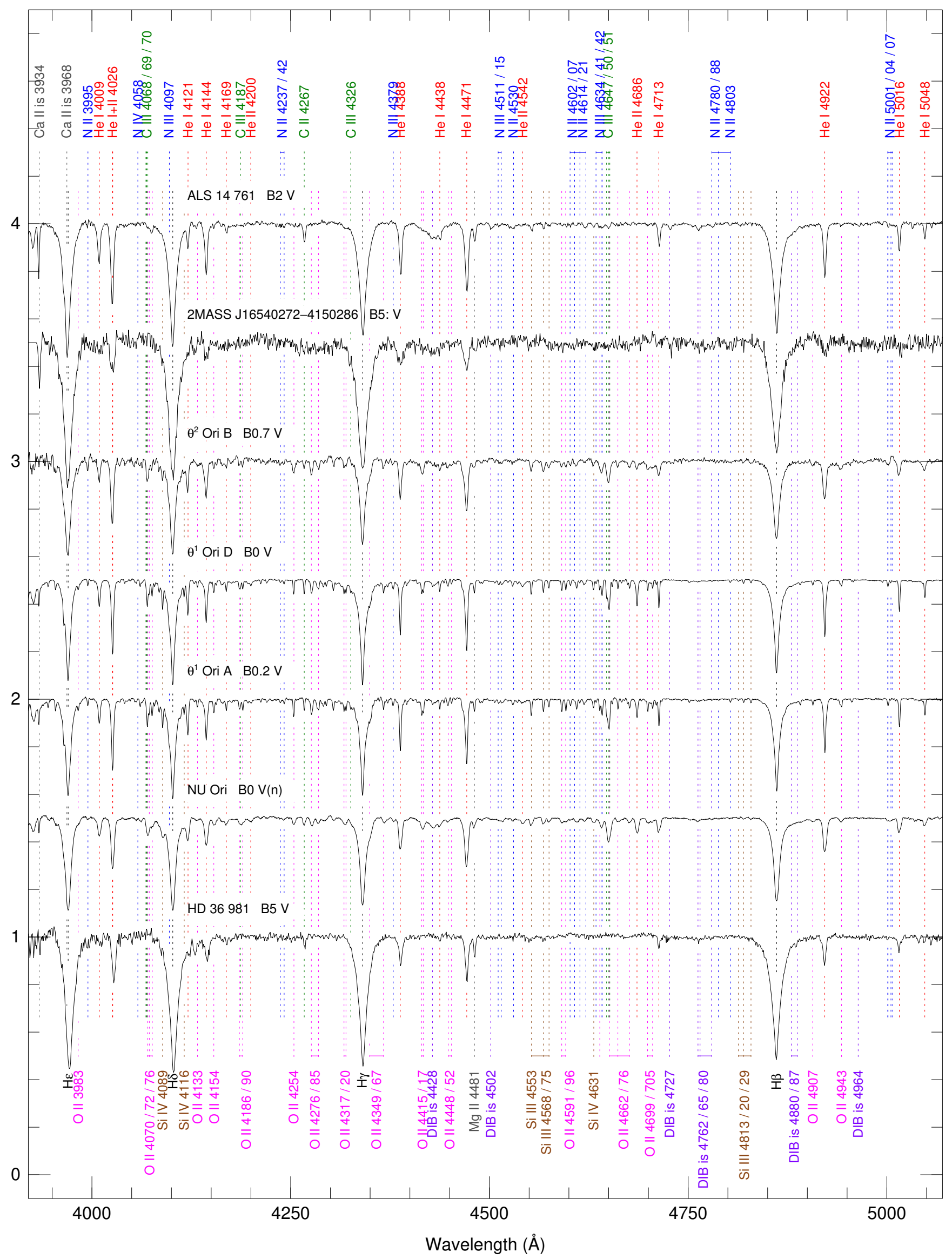

Fig. A.13. New GOSSS spectra for stars in Villafranca O-022 and Villafranca O-023. 
A\&A 657, A131 (2022)

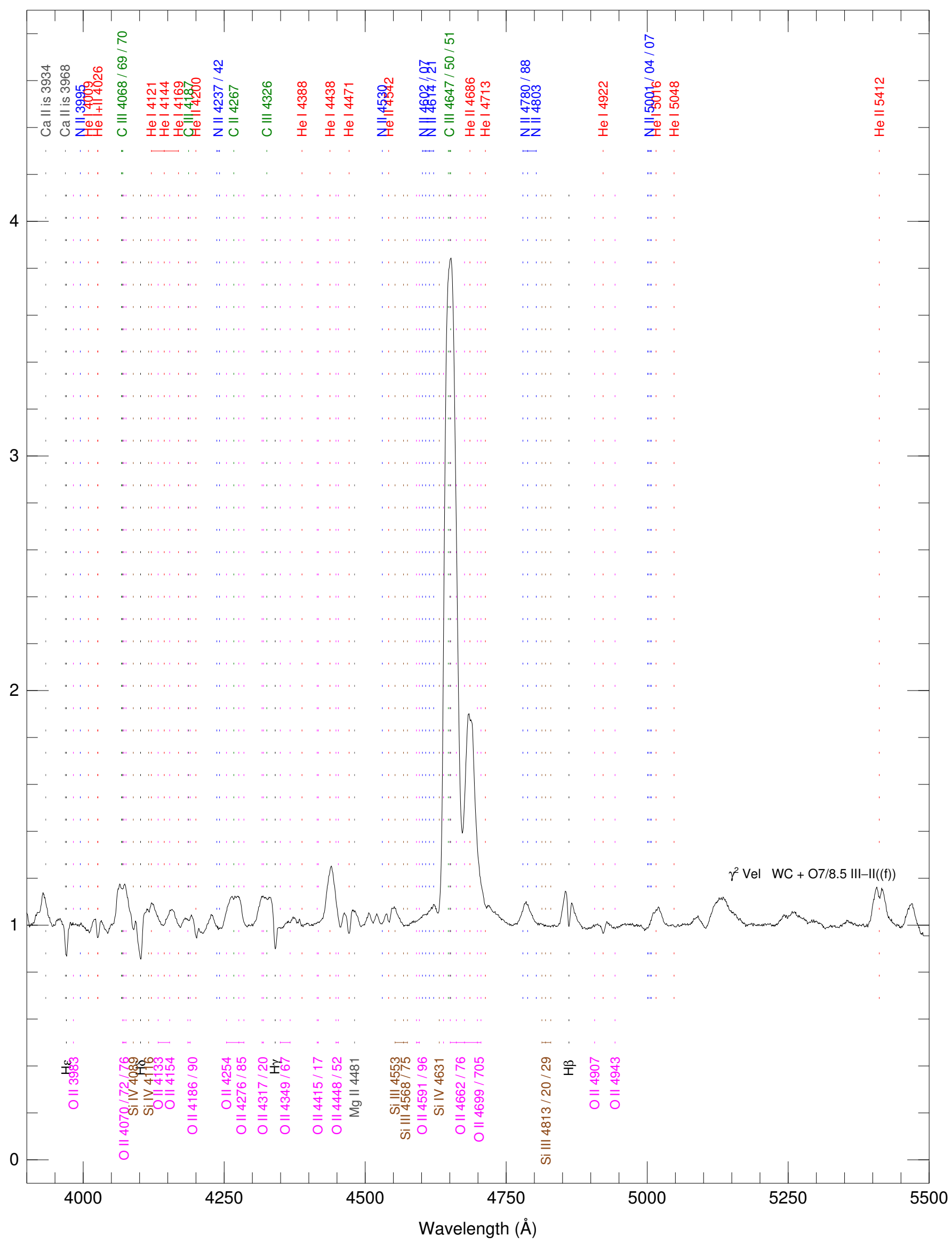

Fig. A.14. New GOSSS spectra for a star in Villafranca O-024. Note the expanded wavelength range. 
J. Maíz Apellániz et al.: Villafranca OB groups. II.

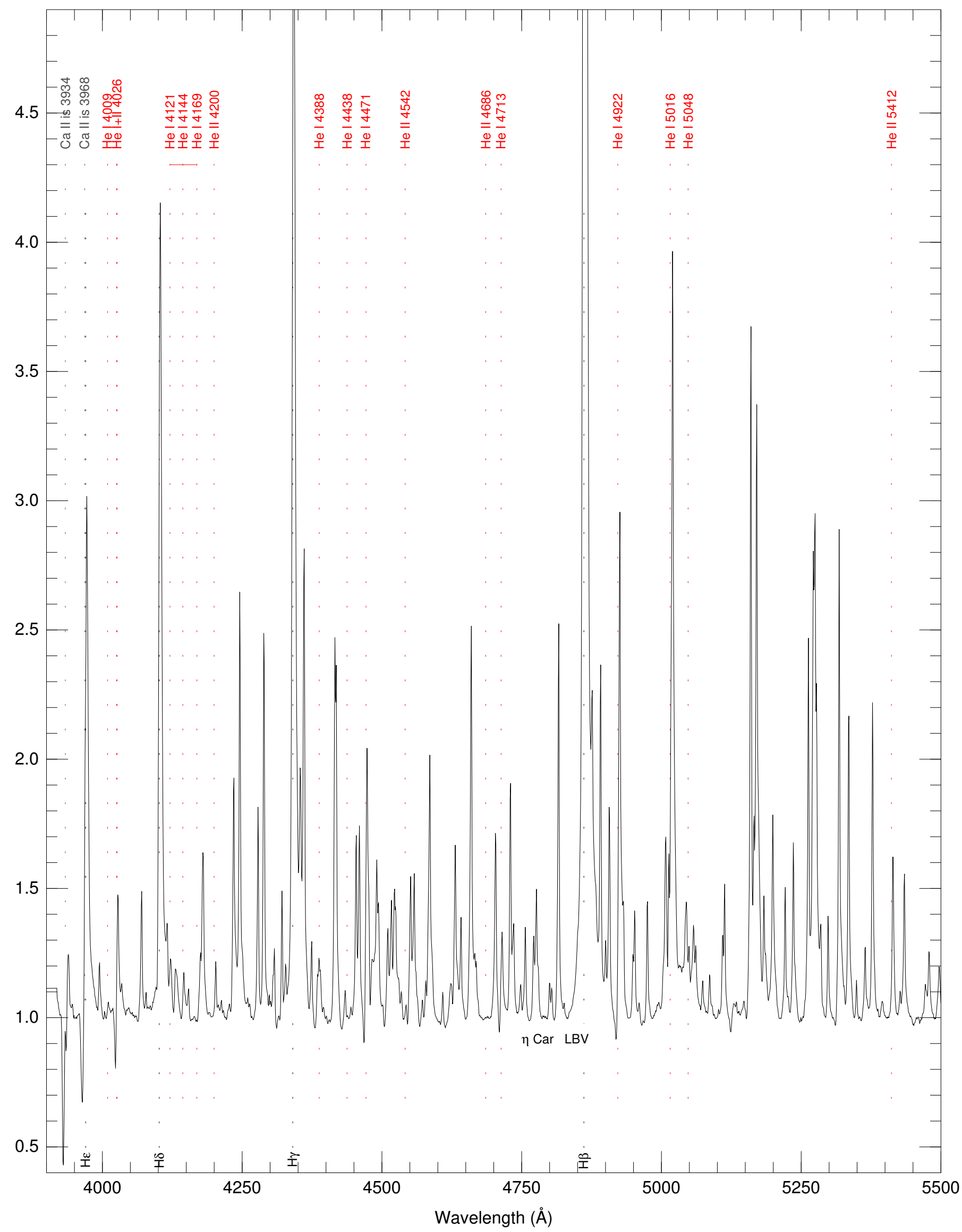

Fig. A.15. New GOSSS spectra for a star in Villafranca O-025. Note the expanded wavelength range. 


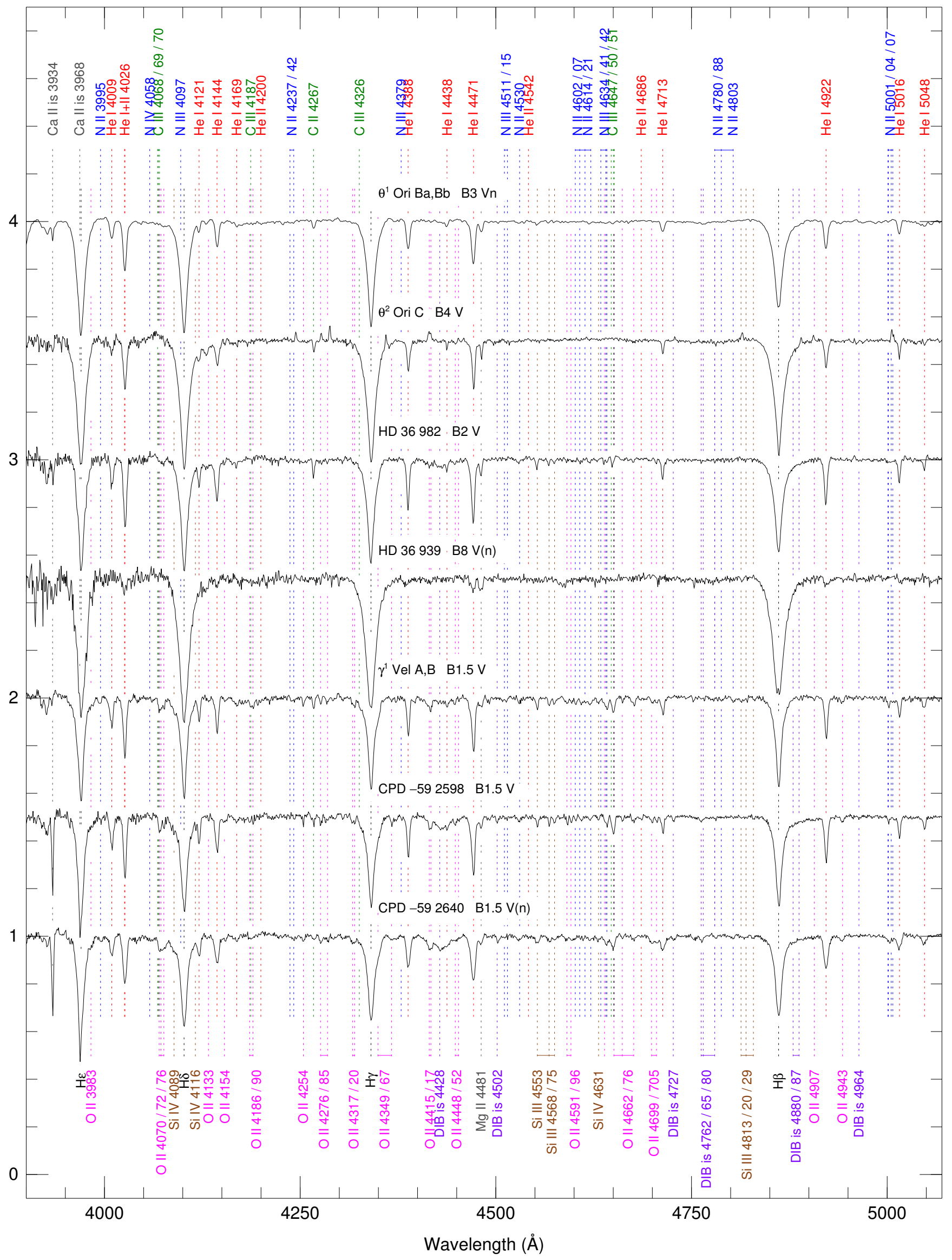

Fig. A.16. New GOSSS spectra for stars in Villafranca O-023, Villafranca O-024, and Villafranca O-025. 
J. Maíz Apellániz et al.: Villafranca OB groups. II.

Table A.3. New GOSSS spectral classificatons.

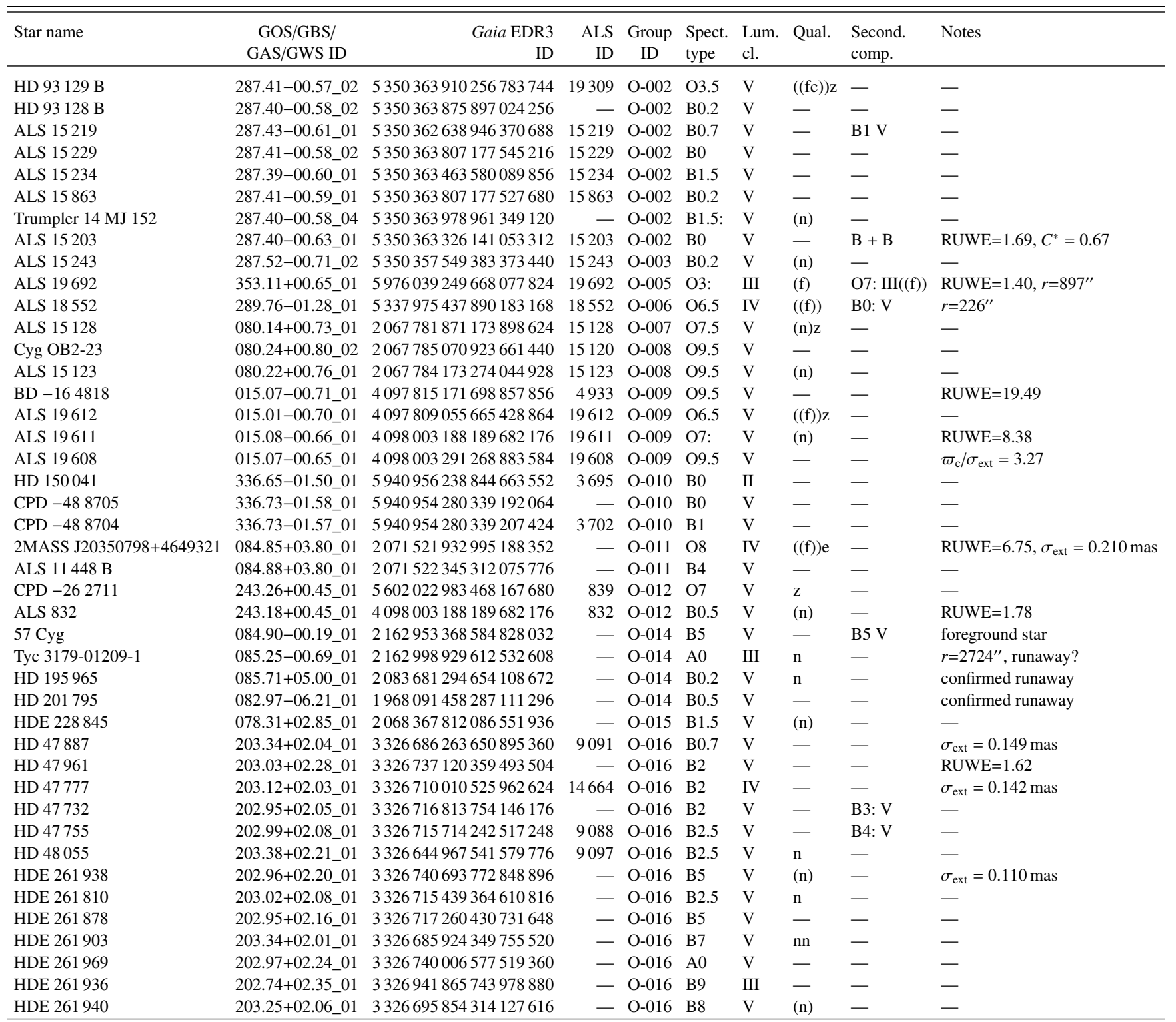


A\&A 657, A131 (2022)

Table A.3. Continued.

\begin{tabular}{|c|c|c|c|c|c|c|c|c|c|}
\hline Star name & $\begin{array}{c}\text { GOS/GBS/ } \\
\text { GAS/GWS ID }\end{array}$ & $\begin{array}{r}\text { Gaia EDR3 } \\
\text { ID }\end{array}$ & $\begin{array}{r}\text { ALS } \\
\text { ID }\end{array}$ & $\begin{array}{l}\text { Group } \\
\text { ID }\end{array}$ & $\begin{array}{l}\text { Spect. } \\
\text { type }\end{array}$ & $\begin{array}{l}\text { Lum. } \\
\text { cl. }\end{array}$ & Qual. & $\begin{array}{l}\text { Second. } \\
\text { comp. }\end{array}$ & Notes \\
\hline BD +60 493 & $134.62+00.58 \_01$ & 465503094013753728 & 7249 & O-017 & B0.5 & Ia & - & - & - \\
\hline $\mathrm{BD}+60496$ & $134.61+00.96 \_01$ & 465533158786645504 & 7265 & $\mathrm{O}-017$ & B0.7 & $\mathrm{V}$ & - & B1 V & - \\
\hline KM Cas & $134.36+00.82 \_01$ & 465523778576137600 & 7225 & O-017 & 09.2 & IV & $\mathrm{n}$ & - & - \\
\hline ALS 7320 & $134.91+00.95 \_01$ & 465487322893075840 & 7320 & O-017 & B 0.7 & $\mathrm{~V}$ & - & B1.5: $\mathrm{V}$ & - \\
\hline V1166 Cas & 134.59+01.07_01 & 465551472526065152 & 7268 & O-017 & B1 & $\mathrm{V}$ & - & - & - \\
\hline ALS 7299 & $134.78+00.95 \_01$ & 465533742902211072 & 7299 & O-017 & B 0.7 & $\mathrm{~V}$ & - & - & RUWE $=1.51$ \\
\hline ALS 7270 & $134.70+00.8101$ & 465526596075683072 & 7270 & O-017 & B & - & $\mathrm{e}$ & - & RUWE $=3.15, r_{\mu}=0.83 \mu$ as $/$ a, also Table A. 4 \\
\hline ALS 15320 & $134.77+00.95 \_01$ & 465534498815432704 & 15320 & O-017 & B2.5 & $\mathrm{V}$ & $\mathrm{nn}$ & - & - \\
\hline HD 164906 & $006.05-01.33-01$ & 4065974968548020352 & 4616 & O-018 & B & - & $\mathrm{e}$ & - & - \\
\hline HDE 315031 & 006.07-01.32_01 & 4065975002907765760 & 4618 & $\mathrm{O}-018$ & B1 & $\mathrm{V}$ & - & - & - \\
\hline CPD -246140 & 005.99-01.18_01 & 4066021182400931072 & 4592 & $\mathrm{O}-018$ & B1.5 & $\mathrm{V}$ & (n) & - & - \\
\hline BD -134930 & 016.94+00.77_01 & 4146599476126383872 & 9505 & O-019 & O9.7 & $\mathrm{V}$ & - & - & - \\
\hline BD - 134929 & 016.98+00.87_01 & 4146600781797073920 & 4913 & O-019 & O8 & $\mathrm{V}$ & - & $B 0.5: V+B 0.5: V$ & RUWE $=1.75$, also Table A. 4 \\
\hline BD -13 4921 & $016.90+00.85 \_01$ & 4146598995090108288 & 9491 & O-019 & B0.7 & $\mathrm{V}$ & - & - & - \\
\hline BD -134928 & $016.97+00.82 \_01$ & 4146600678717290624 & 4911 & O-019 & 09.7 & IV & $\mathrm{nn}$ & - & also Table A.4 \\
\hline ALS 4903 & $016.92+00.85 \_01$ & 4146599029451913984 & 4903 & O-019 & O9 & $\mathrm{V}$ & - & - & - \\
\hline ALS 15352 & 017.00+00.90_01 & 4146613632338722432 & 15352 & O-019 & O8 & IV & - & - & - \\
\hline ALS 15369 & $016.98+00.8401$ & 4146612425449995648 & 15369 & O-019 & B 0.5 & $\mathrm{~V}$ & - & - & - \\
\hline ALS 15363 & 016.99+00.86_01 & 4146612803413634688 & 15363 & O-019 & B1.5 & $\mathrm{V}$ & $\mathrm{p}$ & - & - \\
\hline ALS 15360 & $017.00+00.8701$ & 4146612872133107584 & 15360 & O-019 & O6.5 & $\mathrm{V}$ & ((f)) $\mathrm{z}$ & - & - \\
\hline HDE 259135 & 206.37-02.08_01 & 3131334452194616192 & 8986 & $\mathrm{O}-020$ & B0 & $\mathrm{V}$ & - & B1: V & also Table A.4 \\
\hline HDE 259481 & 206.43-01.84_01 & 3131332940366123392 & 9001 & $\mathrm{O}-020$ & B1.5: & $\mathrm{V}$ & (n)e & - & RUWE $=1.66, r_{\mu}=1.02 \mu \mathrm{as} / \mathrm{a}$ \\
\hline HDE 258691 & 206.37-02.49_01 & 3131301299346222080 & 8970 & $\mathrm{O}-020$ & O9.5 & $\mathrm{V}$ & - & - & RUWE $=6.97, r=1517^{\prime \prime}$, runaway?, also Table A.4 \\
\hline HD 46056 B & 206.33-02.25_01 & 3131327653265431552 & - & $\mathrm{O}-020$ & B2 & $\mathrm{V}$ & - & - & - \\
\hline WR 79 & $343.49+01.1601$ & 5966509541883497984 & 3810 & $\mathrm{O}-022$ & WC & - & - & O4 III-I(f) & also Table A.4 \\
\hline HD 152234 & $343.46+01.22 \_01$ & 5966510057279631488 & 15086 & $\mathrm{O}-022$ & B 0.5 & Ia & - & - & RUWE $=4.01, \sigma_{\text {ext }}=1.397 \mathrm{mas}$, also Table A. 4 \\
\hline HD 152235 & $343.31+01.10-01$ & 5966501501704611840 & 3807 & $\mathrm{O}-022$ & B0.7 & Ia & - & - & $\sigma_{\mathrm{ext}}=0.117 \mathrm{mas}$ \\
\hline HD 152076 & 343.42+01.40_01 & 5966520876291200256 & 3794 & $\mathrm{O}-022$ & B0 & III & - & - & - \\
\hline CPD -417723 & $343.45+01.19-01$ & 5966509816761424256 & 16063 & $\mathrm{O}-022$ & B1.5 & $\mathrm{V}$ & - & - & - \\
\hline CPD -41 7724 & 343.46+01.19_01 & 5966509846818671872 & 16493 & $\mathrm{O}-022$ & B0.7 & $\mathrm{V}$ & - & - & - \\
\hline HDE 326330 & $343.45+01.1401$ & 5966508957767911040 & 15969 & $\mathrm{O}-022$ & B1 & IV & (n) & - & - \\
\hline HDE 326333 & 343.53+01.10_01 & 5966507823896515200 & 3822 & $\mathrm{O}-022$ & B 0.5 & $\mathrm{~V}$ & - & - & - \\
\hline V945 Sco & 343.40+01.19_01 & 5966504044325373440 & 16119 & $\mathrm{O}-022$ & B1 & III & $\mathrm{n}$ & - & - \\
\hline HDE 326320 & 343.58+01.33_01 & 5966523938614083712 & 16057 & $\mathrm{O}-022$ & B1.5: & $\mathrm{V}$ & - & - & - \\
\hline CPD -417721 B & 343.44+01.18_01 & 5966503941246146176 & - & $\mathrm{O}-022$ & B1.5 & $\mathrm{V}$ & - & - & - \\
\hline ALS 14761 & $343.45+01.12 \_01$ & 5966508717249708672 & 14761 & $\mathrm{O}-022$ & B2 & $\mathrm{V}$ & - & - & - \\
\hline 2MASS J16540272-4150286 & 343.44+01.19_01 & 5966503936943654912 & - & $\mathrm{O}-022$ & B5: & $\mathrm{V}$ & - & - & - \\
\hline$\theta^{2}$ Ori B & 209.06-19.36_01 & 3017360833515044480 & 16182 & $\mathrm{O}-023$ & B0.7 & $\mathrm{V}$ & - & - & $\sigma_{\text {ext }}=0.133$ mas, also Table A. 4 \\
\hline$\theta^{1}$ Ori D & 209.01-19.38_03 & 3017364063330465152 & 16710 & $\mathrm{O}-023$ & B0 & $\mathrm{V}$ & - & - & also Table A.4 \\
\hline$\theta^{1}$ Ori A & 209.01-19.39_01 & 3017364132050194688 & 14651 & $\mathrm{O}-023$ & B 0.2 & $\mathrm{~V}$ & - & - & RUWE $=1.49, \sigma_{\text {ext }}=0.279$ mas, also Table A. 4 \\
\hline NU Ori & 208.92-19.27_01 & 3017367396223983616 & 16712 & $\mathrm{O}-023$ & B0 & $\mathrm{V}$ & (n) & - & RUWE $=2.41, \sigma_{\text {ext }}=0.226 \mathrm{mas}$, also Table A. 4 \\
\hline HD 36981 & 208.82-19.34_01 & 3209522411971821824 & - & $\mathrm{O}-023$ & B5 & $\mathrm{V}$ & - & - & - \\
\hline$\theta^{1}$ Ori $\mathrm{Ba}, \mathrm{Bb}$ & 209.01-19.38_02 & 3017364132049943680 & 14652 & $\mathrm{O}-023$ & B3 & $\mathrm{V}$ & $\mathrm{n}$ & - & RUWE $=1.46, \sigma_{\text {ext }}=0.146$, also Table A. 4 \\
\hline$\theta^{2}$ Ori C & 209.07-19.34_01 & 3017360799155290368 & - & $\mathrm{O}-023$ & B4 & $\mathrm{V}$ & - & - & $r_{\mu}=3.39 \mu$ as/a, also Table A. 4 \\
\hline HD 36982 & 209.07-19.44_01 & 3017360348171372672 & 16708 & $\mathrm{O}-023$ & B2 & $\mathrm{V}$ & - & - & also Table A.4 \\
\hline HD 36939 & 209.08-19.52_01 & 3017265961968363904 & - & $\mathrm{O}-023$ & B8 & $\mathrm{V}$ & (n) & - & \\
\hline$\gamma^{1}$ Vel A,B & 262.81-07.70_01 & 5519266900766220800 & 14916 & $\mathrm{O}-024$ & B1.5 & $\mathrm{V}$ & - & - & $\sigma_{\text {ext }}=0.338 \mathrm{mas}$ \\
\hline$\gamma^{2} \mathrm{Vel}$ & 262.80-07.69_01 & - & 980 & $\mathrm{O}-024$ & WC & - & - & O7/8.5 III-II((f)) & not in Gaia EDR3, also Table A.4 \\
\hline$\eta \mathrm{Car}$ & $287.60-00.63 \_01$ & 5350358584482202880 & 1868 & $\mathrm{O}-025$ & LBV & - & - & - & no parallax in Gaia EDR3 \\
\hline CPD -59 2598 & 287.56-00.66_01 & 5350358859375074816 & 1854 & $\mathrm{O}-025$ & B1.5 & $\mathrm{V}$ & - & - & - \\
\hline CPD -592640 & 287.61-00.60_01 & 5350381914760913024 & 15223 & $\mathrm{O}-025$ & B1.5 & $\mathrm{V}$ & (n) & - & - \\
\hline CPD -59 2616 & 287.61-00.67_01 & 5350357931646616448 & 19745 & $\mathrm{O}-025$ & B2 & $\mathrm{V}$ & (n) & - & - \\
\hline 2MASS J10452265-5942596 & 287.65-00.64_01 & 5350311374212229120 & - & O-025 & B7 & $\mathrm{V}$ & (n)e & - & RUWE=1.92 \\
\hline$\sigma$ Ori $\mathrm{D}$ & 206.82-17.33_01 & 3216486478101982592 & 8474 & $\mathrm{O}-026$ & B2 & $\mathrm{V}$ & (n) & - & $\sigma_{\mathrm{ext}}=0.160$ mas, also Table A. 4 \\
\hline$\sigma$ Ori E & 206.82-17.32_01 & 3216486478101981056 & 8475 & $\mathrm{O}-026$ & B2 & $\mathrm{V}$ & $\mathrm{p}$ & - & $\sigma_{\mathrm{ext}}=0.168 \mathrm{mas}$, also Table A. 4 \\
\hline HDE 294271 & $206.76-17.34-01$ & 3216489776637339136 & - & O-026 & B5 & $\mathrm{V}$ & (n) & - & RUWE $=1.44$ \\
\hline
\end{tabular}


J. Maíz Apellániz et al.: Villafranca OB groups. II.

Table A.4. New LiLiMaRlin spectral classifications.

\begin{tabular}{|c|c|c|c|c|c|c|c|c|c|}
\hline Star name & $\begin{array}{c}\text { GOS/GBS/ } \\
\text { GAS/GWS ID }\end{array}$ & $\begin{array}{r}\text { Gaia EDR3 } \\
\text { ID }\end{array}$ & $\begin{array}{r}\text { ALS } \\
\text { ID } \\
\end{array}$ & $\begin{array}{l}\text { Group } \\
\text { ID }\end{array}$ & $\begin{array}{l}\text { Spect. } \\
\text { type }\end{array}$ & $\begin{array}{l}\text { Lum. } \\
\text { cl. }\end{array}$ & Qual. & $\begin{array}{l}\text { Second. } \\
\text { comp. }\end{array}$ & Notes \\
\hline NGC 3603 HST-5 & 291.62-00.52_36 & 5337418397799185536 & - & O-001 & B1.5 & Ia & - & - & - \\
\hline HD $93161 \mathrm{~A}$ & 287.44-00.59_02 & 5350362982543827456 & 1832 & $\mathrm{O}-002$ & O7 & $\mathrm{V}$ & $((\mathrm{f}))$ & O9 IV & also Sota et al. (2014) \\
\hline ALS 7270 & 134.70+00.81_01 & 465526596075683072 & 7270 & O-017 & B & - & $\mathrm{e}$ & - & RUWE $=3.15, r_{\mu}=0.83 \mu$ as/a, also Table A. 3 \\
\hline HD 164816 & 006.06-01.20_01 & 4066023415781257984 & 4597 & O-018 & 09.5 & $\mathrm{~V}$ & - & $09.7 \mathrm{~V}$ & also Sota et al. (2014) \\
\hline HD 165052 & 006.12-01.48_01 & 4066064956700837248 & 4635 & O-018 & 06.5 & $\mathrm{~V}$ & $((\mathrm{f})) \mathrm{z}$ & $06.5 \mathrm{~V}((\mathrm{f})) \mathrm{z}$ & also Maíz Apellániz et al. (2016) \\
\hline BD -134930 & 016.94+00.77_01 & 4146599476126383872 & 9505 & O-019 & 09.7 & $\mathrm{~V}$ & - & - & also Table A.3 \\
\hline BD -134929 & 016.98+00.87_01 & 4146600781797073920 & 4913 & O-019 & O8 & $\mathrm{V}$ & - & $B 0.5: V+B 0.5: V$ & RUWE $=1.75$, also Table A. 3 \\
\hline BD -134928 & $016.97+00.82 \_01$ & 4146600678717290624 & 4911 & O-019 & 09.7 & IV & $\mathrm{nn}$ & - & also Table A. 3 \\
\hline HD 46106 & 206.20-02.09_01 & 3131384484272514688 & 8981 & $\mathrm{O}-020$ & 09.7 & $\mathrm{~V}$ & - & B2: Vn & also Sota et al. (2014) \\
\hline HDE 259135 & 206.37-02.08_01 & 3131334452194616192 & 8986 & $\mathrm{O}-020$ & B0 & $\mathrm{V}$ & - & B1: V & also Table A.3 \\
\hline HDE 258691 & 206.37-02.49_01 & 3131301299346222080 & 8970 & $\mathrm{O}-020$ & 09.5 & $\mathrm{~V}$ & - & - & RUWE $=6.97, r=1517^{\prime \prime}$, runaway?, also Table A.3 \\
\hline HD 152234 & $343.46+01.22 \_01$ & 5966510057279631488 & 15086 & $\mathrm{O}-022$ & B0.5 & Ia & - & - & RUWE $=4.01, \sigma_{\text {ext }}=1.397$ mas, also Table A. 3 \\
\hline $\mathrm{HD} 152248 \mathrm{Aa}, \mathrm{Ab}$ & 343.46+01.18_01 & 5966509885480902656 & 15087 & O-022 & O7 & $\mathrm{Ib}$ & (f) & $\mathrm{O} 7 \mathrm{Ib}(\mathrm{f})$ & also Sota et al. (2014) \\
\hline HD 152218 & 343.53+01.28_01 & 5966522667303699072 & 15085 & O-022 & 08.5 & IV & - & B0 IV & also Sota et al. (2014) \\
\hline WR 79 & 343.49+01.16_01 & 5966509541883497984 & 3810 & O-022 & WC7 & - & - & O4 III-I(f) & also Table A.3 \\
\hline CPD -41 7733 & 343.46+01.17_01 & 5966509022176616448 & 15756 & O-022 & O9 & IV & - & B & also Sota et al. (2014) \\
\hline V1034 Sco & $343.48+01.15 \_01$ & 5966509095207261056 & 15757 & O-022 & 09.5 & IV & - & B1: V & also Maíz Apellániz et al. (2016) \\
\hline$\theta^{2}$ Ori B & 209.06-19.36_01 & 3017360833515044480 & 16182 & $\mathrm{O}-023$ & B0.7 & V & - & - & $\sigma_{\text {ext }}=0.133$ mas, also Table A. 3 \\
\hline$\theta^{1}$ Ori D & 209.01-19.38_03 & 3017364063330465152 & 16710 & O-023 & B0 & $\mathrm{V}$ & - & - & also Table A.3 \\
\hline$\theta^{1}$ Ori A & 209.01-19.39_01 & 3017364132050194688 & 14651 & $\mathrm{O}-023$ & B 0.2 & $\mathrm{~V}$ & - & - & RUWE $=1.49, \sigma_{\text {ext }}=0.279$ mas, also Table A. 3 \\
\hline NU Ori & 208.92-19.27_01 & 3017367396223983616 & 16712 & $\mathrm{O}-023$ & B0 & $\mathrm{V}$ & (n) & - & RUWE $=2.41, \sigma_{\text {ext }}=0.226$ mas, also Table A. 3 \\
\hline$\theta^{1}$ Ori $\mathrm{Ba}, \mathrm{Bb}$ & 209.01-19.38_02 & 3017364132049943680 & 14652 & $\mathrm{O}-023$ & B3 & $\mathrm{V}$ & $\mathrm{n}$ & - & RUWE $=1.46, \sigma_{\text {ext }}=0.146$ mas, also Table A. 3 \\
\hline$\theta^{2}$ Ori C & 209.07-19.34_01 & 3017360799155290368 & - & $\mathrm{O}-023$ & B4 & $\mathrm{V}$ & - & - & $r_{\mu}=3.39 \mu$ as $/ \mathrm{a}$, also Table A. 3 \\
\hline HD 36982 & 209.07-19.44_01 & 3017360348171372672 & 16708 & $\mathrm{O}-023$ & $\mathrm{~B} 2$ & $\mathrm{~V}$ & - & - & also Table A.3 \\
\hline$\gamma^{2} \mathrm{Vel}$ & 262.80-07.69_01 & - & 980 & $\mathrm{O}-024$ & WC8 & - & - & O7/8.5 III-II((f)) & not in Gaia EDR3, also Table A.3 \\
\hline V572 Car & 287.59-00.69_01 & 5350358069101053184 & 1861 & O-025 & 06.5 & $\mathrm{~V}$ & $\mathrm{z}$ & $\mathrm{B} 0 \mathrm{~V}+\mathrm{B} 0.2 \mathrm{~V}$ & also Maíz Apellániz et al. (2016) \\
\hline CPD -592635 & 287.64-00.68_02 & 5350358069101053184 & 1872 & O-025 & O8 & $\mathrm{V}$ & $\mathrm{z}$ & $09.2 \mathrm{~V}$ & also Sota et al. (2014) \\
\hline HD 93343 & 287.64-00.68_01 & 5350310824456389504 & 16717 & $\mathrm{O}-025$ & 07.5 & $\mathrm{~V}$ & $\mathrm{z}$ & O7.5: V(n) & also Maíz Apellániz et al. (2016) \\
\hline V573 Car & $287.60-00.62 \_01$ & 5350358481418098944 & 1871 & O-025 & 09.5 & IV & - & B $0.5 \mathrm{~V}$ & also Sota et al. (2014) \\
\hline$\sigma$ Ori $\mathrm{D}$ & 206.82-17.33_01 & 3216486478101982592 & 8474 & $\mathrm{O}-026$ & B2 & $\mathrm{V}$ & (n) & - & $\sigma_{\text {ext }}=0.160$ mas, also Table A. 3 \\
\hline$\sigma$ Ori E & 206.82-17.32_01 & 3216486478101981056 & 8475 & O-026 & B2 & $\mathrm{V}$ & $\mathrm{p}$ & - & $\sigma_{\text {ext }}=0.168$ mas, also Table A. 3 \\
\hline
\end{tabular}


A\&A 657, A131 (2022)

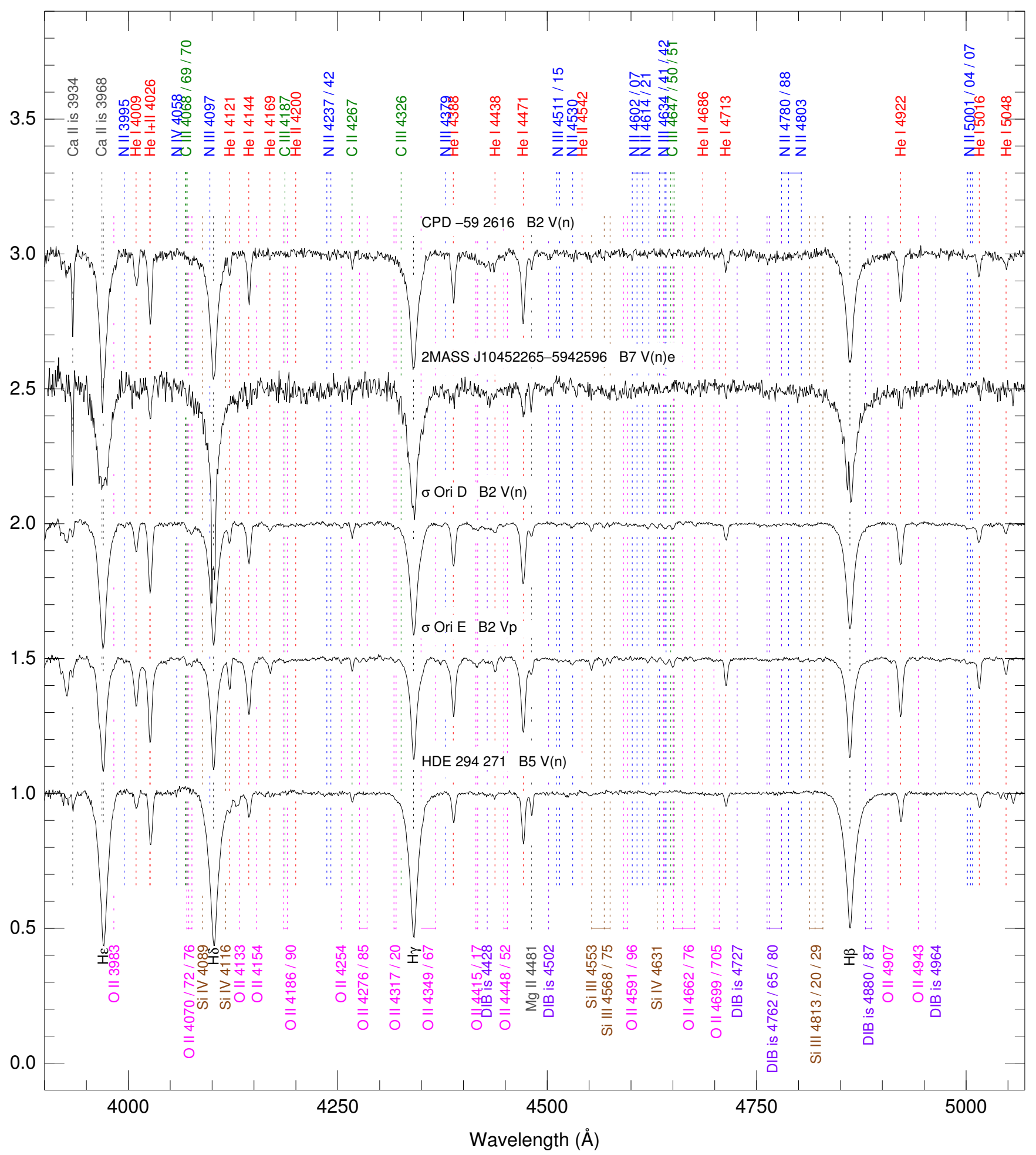

Fig. A.17. New GOSSS spectra for stars in Villafranca O-025 and Villafranca O-026. 
J. Maíz Apellániz et al.: Villafranca OB groups. II.

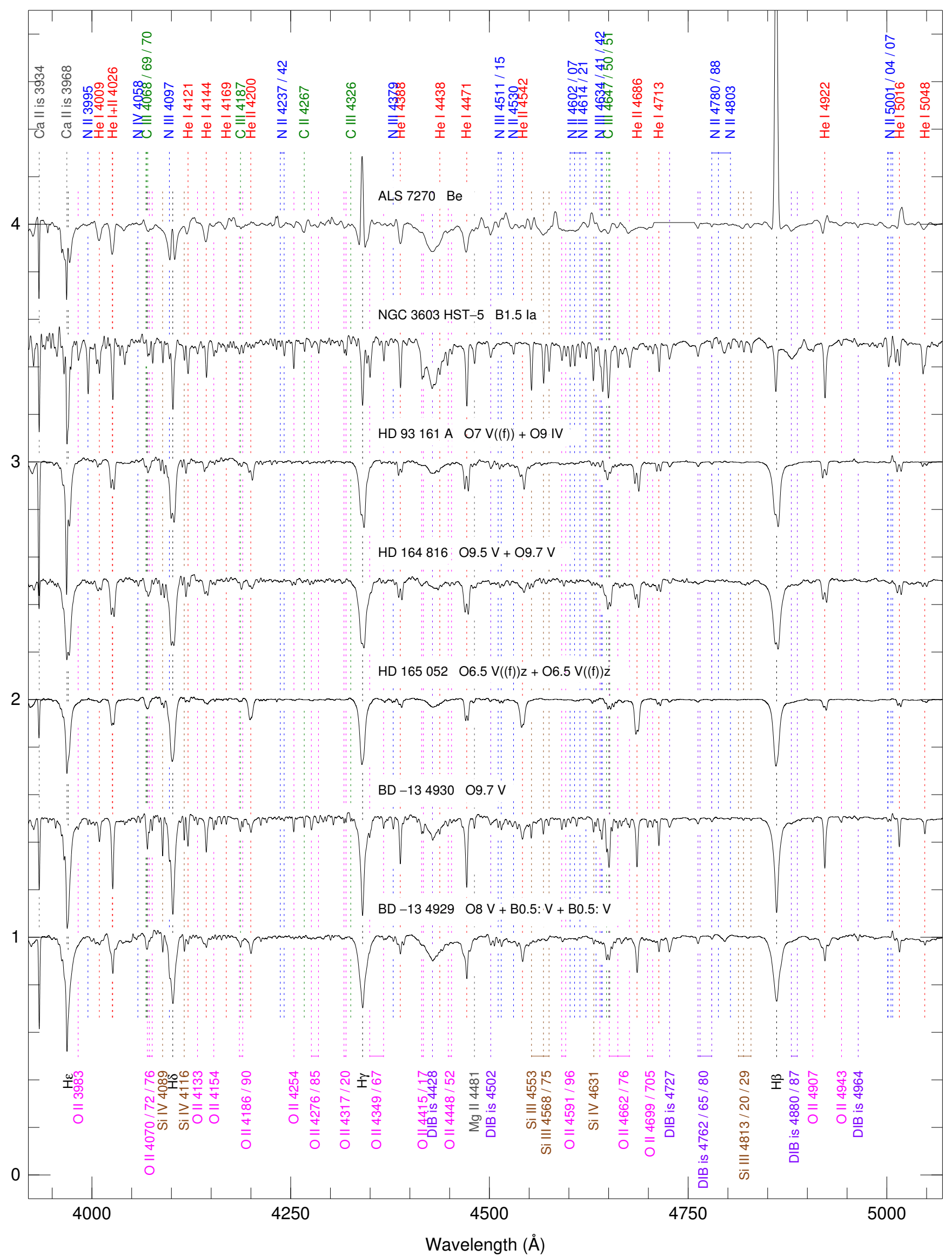

Fig. A.18. New LiLiMaRlin spectra at $R \sim 2500$ for stars in Villafranca O-001, Villafranca O-002, Villafranca O-018, and Villafranca O-019. Note that the ALS 7270 data does not include the 4700-4760 A region. 


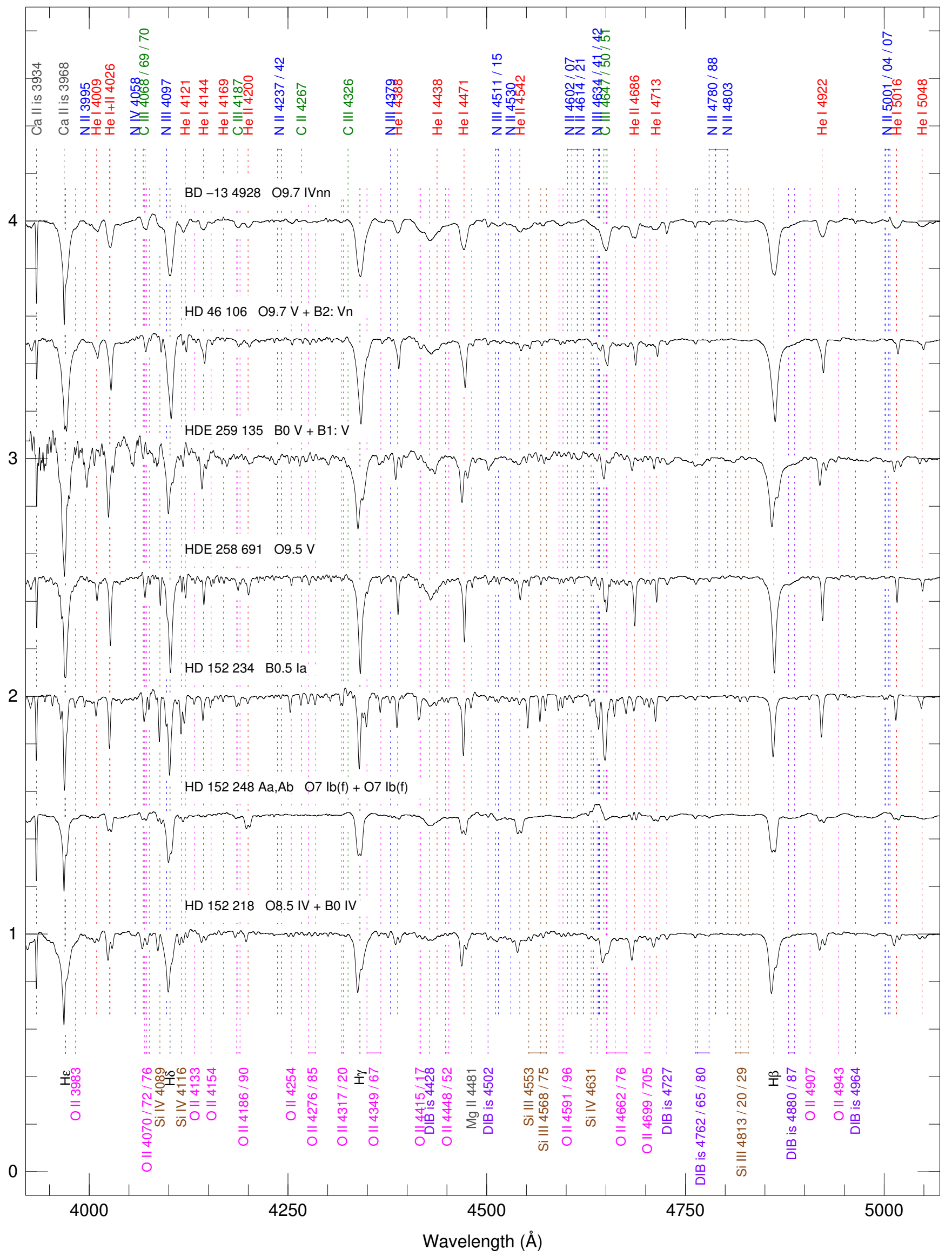

Fig. A.19. New LiLiMaRlin spectra at $R \sim 2500$ for stars in Villafranca O-019, Villafranca O-020, and Villafranca O-022. 
J. Maíz Apellániz et al.: Villafranca OB groups. II.

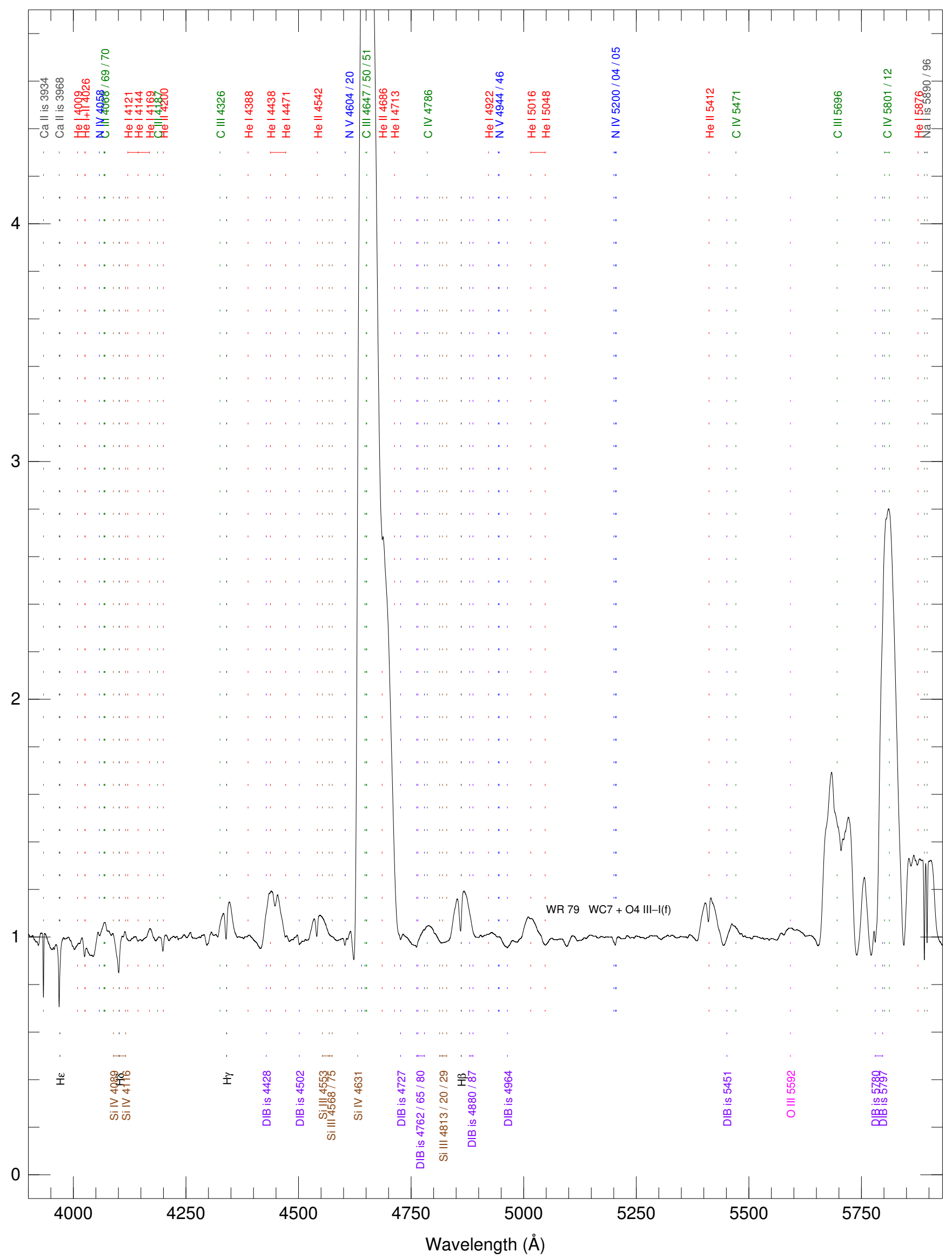

Fig. A.20. New LiLiMaRlin spectra at $R \sim 2500$ for a star in Villafranca O-022. Note the expanded wavelength range. 


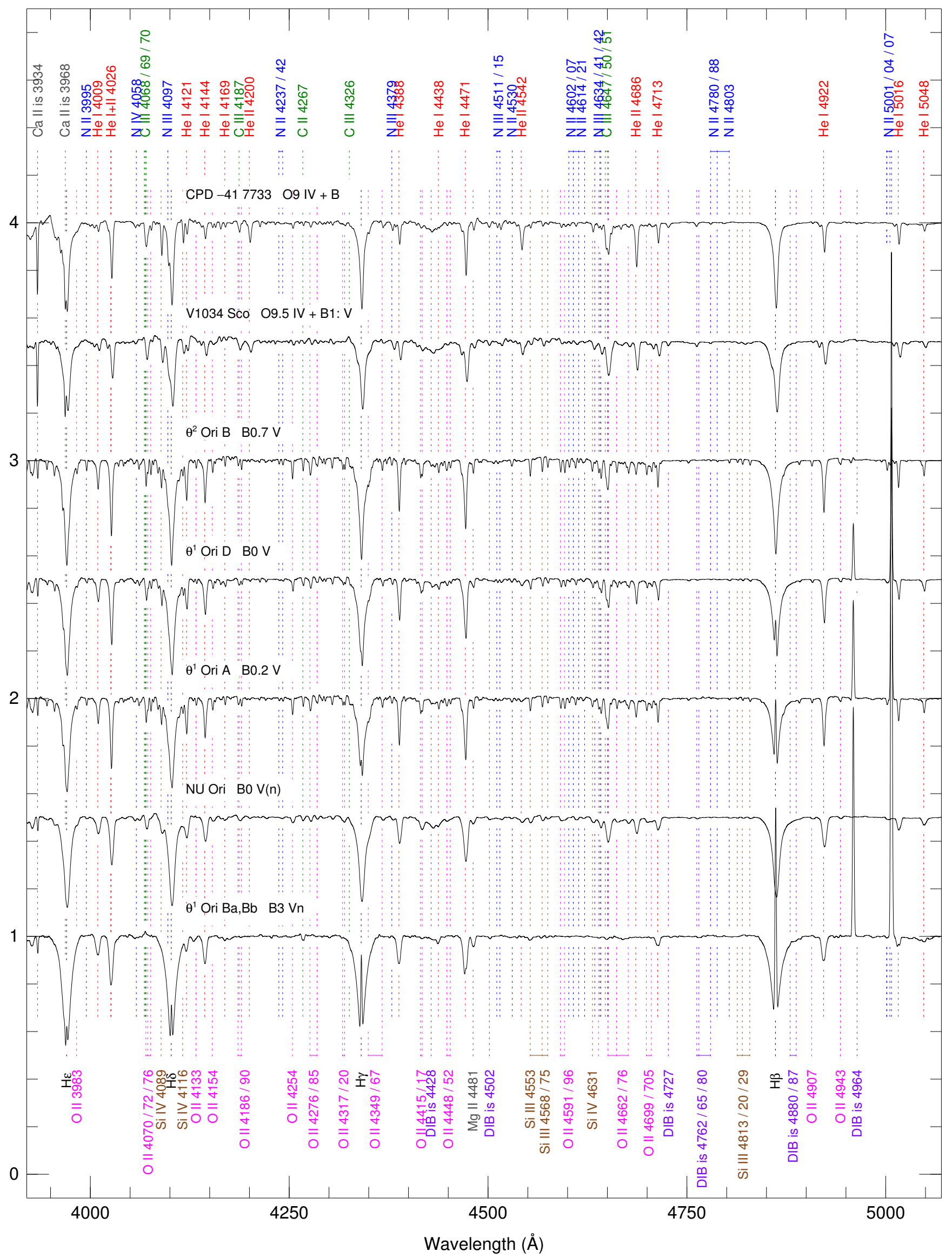

Fig. A.21. New LiLiMaRlin spectra at $R \sim 2500$ for stars in Villafranca O-022 and Villafranca O-023. 
J. Maíz Apellániz et al.: Villafranca OB groups. II.

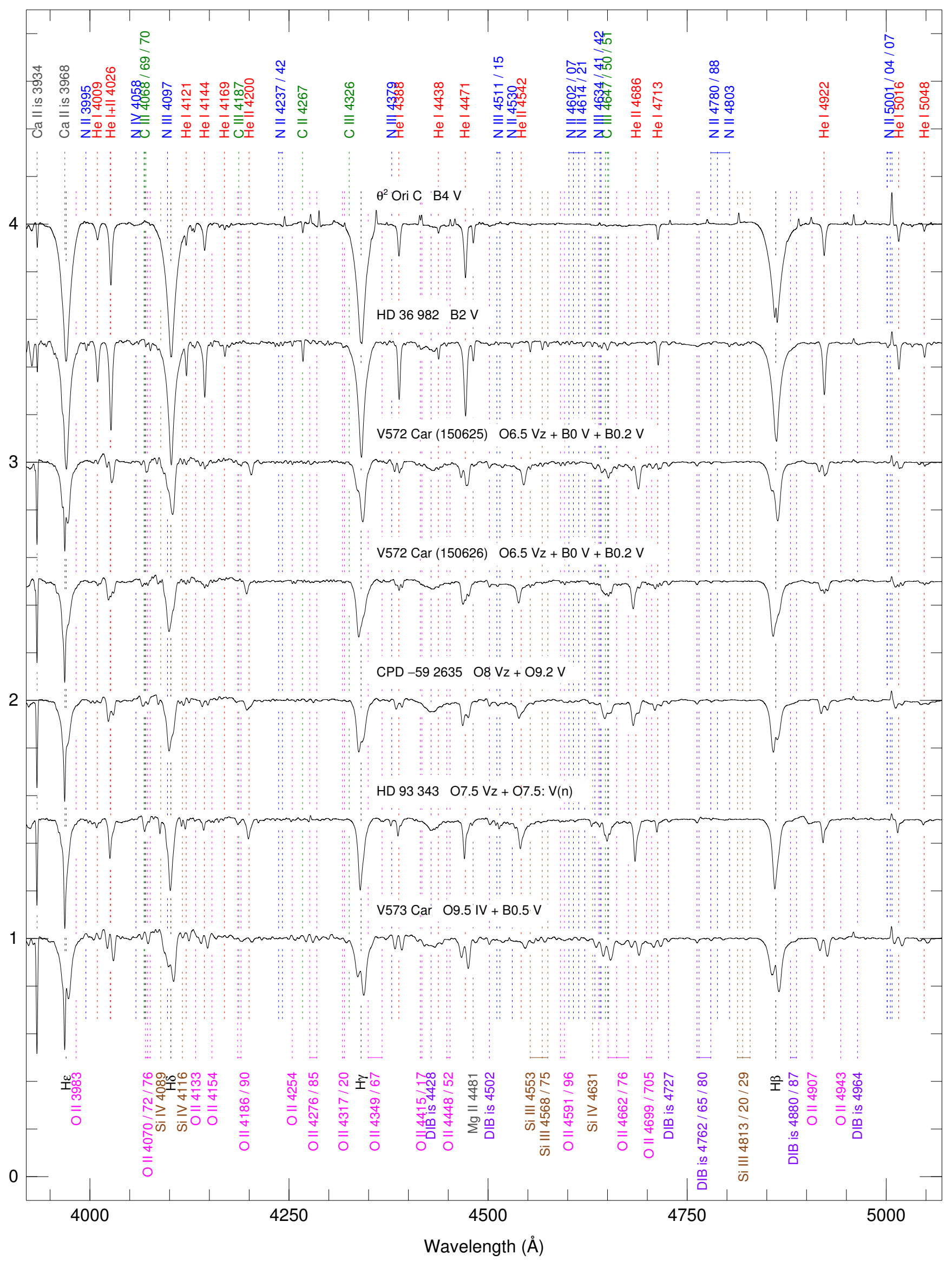

Fig. A.22. New LiLiMaRlin spectra at $R \sim 2500$ for stars in Villafranca O-023, Villafranca O-025, and Villafranca O-026. 
A\&A 657, A131 (2022)

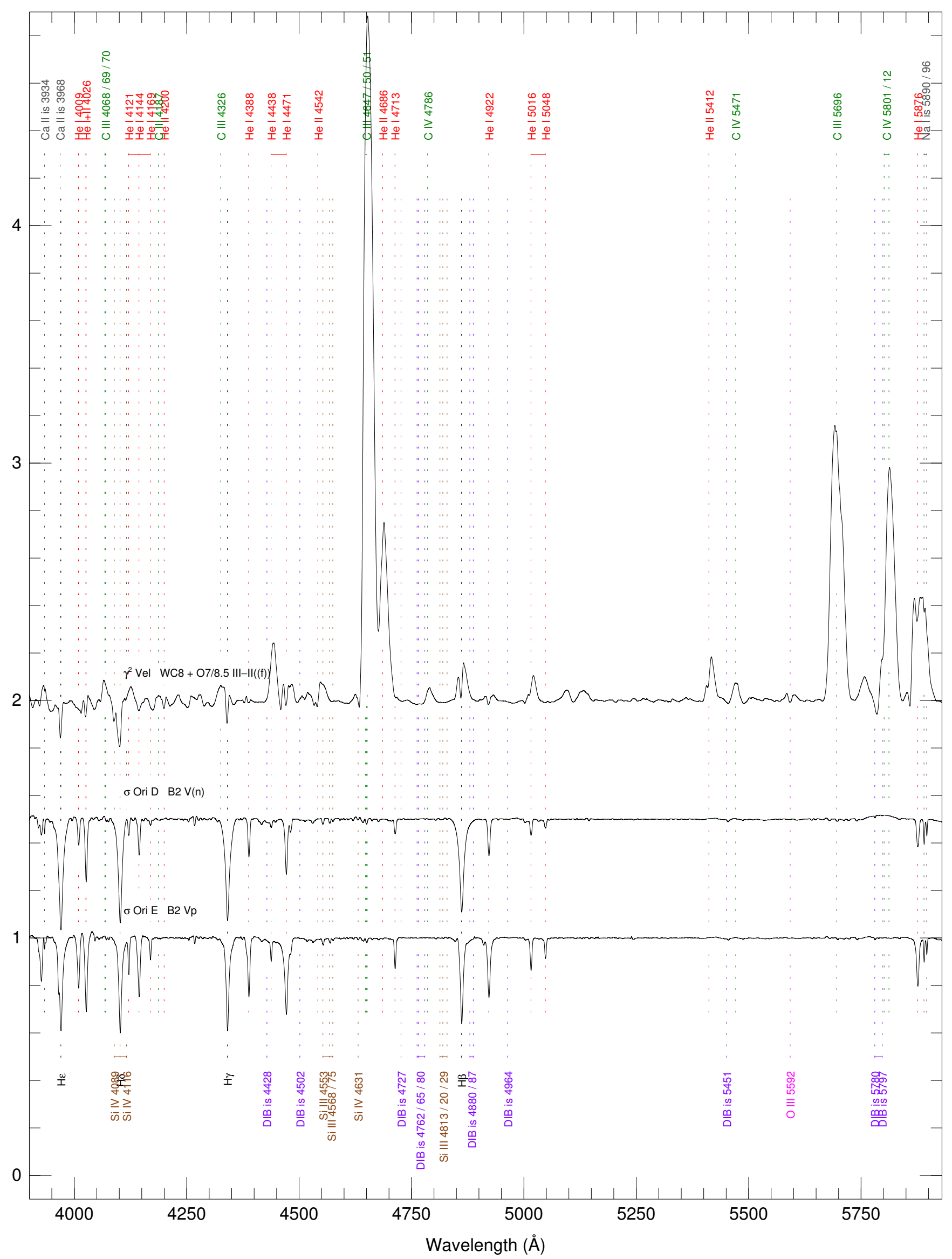

Fig. A.23. New LiLiMaRlin spectra at $R \sim 2500$ for stars in Villafranca O-024 and Villafranca O-026. Note the expanded wavelength range. 